\title{
Almost Periodic Solution of a Multispecies Discrete Mutualism System with Feedback Controls
}

\author{
Hui Zhang, ${ }^{1}$ Feng Feng, ${ }^{2}$ Bin Jing, ${ }^{1}$ and Yingqi Li ${ }^{1}$ \\ ${ }^{1}$ Mathematics and OR Section, Xi'an Research Institute of High-Tech Hongqing Town, Xian, Shaanxi 710025, China \\ ${ }^{2}$ Department of Applied Mathematics, School of Science, Xian University of Posts and Telecommunications, Xian, \\ Shaanxi 710121, China
}

Correspondence should be addressed to Hui Zhang; zh53054958@163.com

Received 5 April 2014; Accepted 27 September 2014

Academic Editor: Guang Zhang

Copyright (C) 2015 Hui Zhang et al. This is an open access article distributed under the Creative Commons Attribution License, which permits unrestricted use, distribution, and reproduction in any medium, provided the original work is properly cited.

\begin{abstract}
We consider an almost periodic multispecies discrete Lotka-Volterra mutualism system with feedback controls. We firstly obtain the permanence of the system by utilizing the theory of difference equation. By means of constructing a suitable Lyapunov function, sufficient conditions are obtained for the existence of a unique positive almost periodic solution which is uniformly asymptotically stable. An example together with numerical simulation indicates the feasibility of the main result.
\end{abstract}

\section{Introduction}

The mutualism system [1] has been studied by more and more scholars. Topics such as permanence, global attractivity, and global stability of continuous differential mutualism system were extensively investigated (see [2-7] and the references cited therein). In addition, some recent attention was on the permanence and global stability of discrete mutualism system, and many excellent results have been derived (see $[3,8-13]$ and the references cited therein).

Recently, the multispecies descrete Lotka-Volterra ecosystem is increasingly concerned (see [12-21] and the references cited therein). Yang and Li [19] studied a discrete nonlinear $\mathrm{N}$-species cooperation system with time delays and feedback controls. Sufficient conditions which ensure the permanence of the system are obtained. Li and Zhang [21] studied a discrete $n$-species cooperation system with time-varying delays and feedback controls. Sufficient conditions are obtained for the permanence of the system.

In real world phenomenon, the environment varies due to the factors such as seasonal effects of weather, food supplies, mating habits, and harvesting. So it is usual to assume the periodicity of parameters in the systems. However, if the various constituent components of the temporally nonuniform environment are with incommensurable (nonintegral multiples) periods, then one has to consider the environment to be almost periodic since there is no a priori reason to expect the existence of periodic solutions. For this reason, the assumption of almost periodicity is more realistic, more important, and more general when we consider the effects of the environmental factors. In fact, there have been many nice works on the positive almost periodic solutions of continuous and discrete dynamics model with almost periodic coefficients (see $[7,12,13,22-28]$ and the references cited therein).

As we all known, investigating the almost periodic solutions of discrete population dynamics model with feedback control has more extensively practical application value (see $[11,22,23,29-34]$ and the references cited therein). Wang [22] considered a nonlinear single species discrete with feedback control and obtained some sufficient conditions which assure the unique existence and global attractivity of almost positive periodic solution. Niu and Chen [30] studied a discrete Lotka-Volterra competitive system with feedback control and obtain the existence and uniqueness of the almost periodic solution which is uniformly asymptotically stable.

Motivated by above, in this paper, we are concerned with the following multispecies discrete Lotka-Volterra mutualism system with feedback controls

$$
\begin{aligned}
& x_{i}(k+1) \\
& =x_{i}(k) \exp \left\{a_{i}(k)-b_{i}(k) x_{i}(k)\right.
\end{aligned}
$$




$$
\begin{gathered}
+\sum_{j=1, j \neq i}^{n} c_{i j}(k) \frac{x_{j}(k)}{d_{i j}(k)+x_{j}(k)} \\
\left.-e_{i}(k) u_{i}(k)\right\}, \\
\Delta u_{i}(k)=-f_{i}(k) u_{i}(k)+\sum_{j=1}^{n} g_{i j}(k) x_{j}(k), \\
i=1,2, \ldots, n,
\end{gathered}
$$

where $\left\{a_{i}(k)\right\},\left\{b_{i}(k)\right\},\left\{c_{i j}(k)\right\},\left\{d_{i j}(k)\right\},\left\{e_{i}(k)\right\},\left\{f_{i}(k)\right\}$ and $\left\{g_{i j}(k)\right\}$ are bounded nonnegative almost periodic sequences such that

$$
\begin{array}{ll}
0<a_{i}^{l} \leq a_{i}(k) \leq a_{i}^{u}, & 0<b_{i}^{l} \leq b_{i}(k) \leq b_{i}^{u}, \\
0<c_{i j}^{l} \leq c_{i j}(k) \leq c_{i j}^{u}, & 0<d_{i j}^{l} \leq d_{i j}(k) \leq d_{i j}^{u}, \\
0<e_{i}^{l} \leq e_{i}(k) \leq e_{i}^{u}, & 0<f_{i}^{l} \leq f_{i}(k) \leq f_{i}^{u}<1, \\
0<g_{i j}^{l} \leq g_{i j}(k) \leq g_{i j}^{u}, &
\end{array}
$$

$i, j=1,2, \ldots, n, k \in \mathbf{Z}$. For any bounded sequence $\{f(k)\}$ defined on $\mathbf{Z}, f^{u}=\sup _{k \in \mathbf{Z}} f(k)$ and $f^{l}=\inf _{k \in \mathbf{Z}} f(k)$.

By the biological meaning, we will focus our discussion on the positive solutions of system (1). So it is assumed that the initial conditions of system (1) are the form:

$$
x_{i}(0)>0, \quad u_{i}(0)>0, \quad i=1,2, \ldots, n .
$$

One can easily show that the solutions of system (1) with the initial condition (3) are defined and remain positive for all $k \in N^{+}=\{0,1,2,3, \ldots\}$.

To the best of our knowledge, this is the first paper to investigate the uniformly asymptotical stability of positive almost periodic solution of multispecies discrete LotkaVolterra mutualism system with feedback controls. The aim of this paper is to obtain sufficient conditions for the existence of a unique uniformly asymptotically stable almost periodic solution of system (1) with initial condition (3), by utilizing the theory of difference equation and constructing a suitable Lyapunov function and applying the analysis technique of papers $[11,22,29,31,32]$.

The remaining part of this paper is organized as follows. In Section 2, we will introduce some definitions and several useful lemmas. In the next section, we establish the permanence of system (1). Then, in Section 4, we establish sufficient conditions to ensure the existence of a unique positive almost periodic solution, which is uniformly asymptotically stable. The main result is illustrated by an example with a numerical simulation in the last section.

\section{Preliminaries}

First, we give the definitions of the terminologies involved.

Definition 1 (see $[35,36]$ ). A sequence $x: Z \rightarrow R$ is called an almost periodic sequence if the $\varepsilon$-translation set of $x$

$$
E\{\varepsilon, x\}=\{\tau \in Z:|x(n+\tau)-x(n)|<\varepsilon, \forall n \in Z\}
$$

is a relatively dense set in $Z$ for all $\varepsilon>0$; that is, for any given $\varepsilon>0$, there exists an integer $l(\varepsilon)>0$ such that each interval of length $l(\varepsilon)$ contains an integer $\tau \in E\{\varepsilon, x\}$ with

$$
|x(n+\tau)-x(n)|<\varepsilon, \quad \forall n \in Z .
$$

$\tau$ is called an $\varepsilon$-translation number of $x(n)$.

Definition 2 (see [37]). Let $D$ be an open subset of $R^{m}, f: Z \times$ $D \rightarrow R^{m} . f(n, x)$ is said to be almost periodic in $n$ uniformly for $x \in D$ if, for any $\varepsilon>0$ and any compact set $S \subset D$, there exists a positive integer $l=l(\varepsilon, S)$ such that any interval of length $l$ contains an integer $\tau$ for which

$$
|f(n+\tau, x)-f(n, x)|<\varepsilon, \quad \forall(n, x) \in Z \times S .
$$

$\tau$ is called an $\varepsilon$-translation number of $f(n, x)$.

Definition 3 (see [38]). The hull of $f$, denoted by $H(f)$, is defined by

$$
\begin{aligned}
H(f)=\{ & g(n, x) \\
& : \lim _{k \rightarrow \infty} f\left(n+\tau_{k}, x\right) \\
& =g(n, x) \text { uniformly on } Z \times S\},
\end{aligned}
$$

for some sequence $\left\{\tau_{k}\right\}$, where $S$ is any compact set in $D$.

Now, we state several lemmas which will be useful in proving our main result.

Lemma 4 (see [39]). $\{x(n)\}$ is an almost periodic sequence if and only if, for any integer sequence $\left\{k_{i}^{\prime}\right\}$, there exists a subsequence $\left\{k_{i}\right\} \subset\left\{k_{i}^{\prime}\right\}$ such that the sequence $\left\{x\left(n+k_{i}\right)\right\}$ converges uniformly for all $n \in Z$ as $i \rightarrow \infty$. Furthermore, the limit sequence is also an almost periodic sequence.

Lemma 5 (see [9]). Assume that $\{x(n)\}$ satisfies $x(n)>0$ and

$$
x(n+1) \leq x(n) \exp \{a(n)-b(n) x(n)\}
$$

for $n \in \mathbf{N}$, where $a(n)$ and $b(n)$ are nonnegative sequences bounded above and below by positive constants. Then

$$
\limsup _{n \rightarrow+\infty} x(n) \leq \frac{1}{b^{l}} \exp \left\{a^{u}-1\right\} .
$$

Lemma 6 (see [9]). Assume that $\{x(n)\}$ satisfies

$$
\begin{gathered}
x(n+1) \geq x(n) \exp \{a(n)-b(n) x(n)\}, \quad n \geq N_{0}, \\
{\limsup x(n) \leq x^{*},}_{n \rightarrow+\infty},
\end{gathered}
$$


and $x\left(N_{0}\right)>0$, where $a(n)$ and $b(n)$ are nonnegative sequences bounded above and below by positive constants and $N_{0} \in N$. Then

$$
\liminf _{n \rightarrow+\infty} x(n) \geq \min \left\{\frac{a^{l}}{b^{u}} \exp \left\{a^{l}-b^{u} x^{*}\right\}, \frac{a^{l}}{b^{u}}\right\} .
$$

Lemma 7 (see [40]). Assume that $A>0$ and $y(0)>1$, and further suppose that

$$
y(n+1) \leq A y(n)+B(n), \quad n=1,2,3, \ldots
$$

Then, for any integer $k \leq n$,

$$
y(n) \leq A^{k} y(n-k)+\sum_{i=0}^{k-1} A^{i} B(n-i-1) .
$$

Specifically, if $A<1$ and $B$ is bounded above with respect to $M$, then

$$
\limsup _{n \rightarrow \infty} y(n) \leq \frac{M}{1-A}
$$

Lemma 8 (see [40]). Assume that $A>0$ and $y(0)>1$, and further suppose that

$$
y(n+1) \geq A y(n)+B(n), \quad n=1,2,3, \ldots
$$

Then, for any integer $k \leq n$,

$$
y(n) \geq A^{k} y(n-k)+\sum_{i=0}^{k-1} A^{i} B(n-i-1) .
$$

Specifically, if $A<1$ and $B$ is bounded below with respect to $m$, then

$$
\liminf _{n \rightarrow \infty} y(n) \geq \frac{m}{1-A} .
$$

Consider the following almost periodic difference system:

$$
x(n+1)=f(n, x(n)), \quad n \in Z^{+},
$$

where $f: Z^{+} \times S_{B} \rightarrow R^{K}, S_{B}=\left\{x \in R^{k}:\|x\|<B\right\}$, and $f(n, x)$ is almost periodic in $n$ uniformly for $x \in S_{B}$ and is continuous in $x$. The product system of (18) is the following system:

$$
x(n+1)=f(n, x(n)), \quad y(n+1)=f(n, y(n)),
$$

and Zhang [38] obtained the following theorem.

Theorem 9 (see [38]). Suppose that there exists a Lyapunov function $V(n, x, y)$ defined for $n \in Z^{+},\|x\|<B$, and $\|y\|<B$ satisfying the following conditions:

(i) $a(\|x-y\|) \leq V(n, x, y) \leq b(\|x-y\|)$, where $a, b \in K$ with $K=\left\{a \in C\left(R^{+}, R^{+}\right): a(0)=\right.$ 0 and $a$ is increasing\};

(ii) $\left\|V\left(n, x_{1}, y_{1}\right)-V\left(n, x_{2}, y_{2}\right)\right\| \leq L\left[\left\|x_{1}-x_{2}\right\|+\left\|y_{1}-y_{2}\right\|\right]$, where $L>0$ is a constant; (iii) $\Delta V_{(19)}(n, x, y) \leq-\alpha V(n, x, y)$, where $0<\alpha<1$ is a constant, and

$$
\Delta V_{(19)}(n, x, y) \equiv V(n+1, f(n, x), f(n, y))-V(n, x, y) \text {. }
$$

Moreover, if there exists a solution $\varphi(n)$ of (18) such that $\|\varphi(n)\| \leq B^{*}<B$ for $n \in Z^{+}$, then there exists a unique uniformly asymptotically stable almost periodic solution $p(n)$ of (18) which is bounded by $B^{*}$. In particular, if $f(n, x)$ is periodic of period $\omega$, then there exists a unique uniformly asymptotically stable periodic solution of (18) of period $\omega$.

\section{Permanence}

In this section, we establish the permanence result for system (1).

Theorem 10. Assume that the conditions (2) and (3) hold; furthermore,

$$
a_{i}^{l}-e_{i}^{u} N_{i}>0,
$$

and then system (1) is permanent; that is, there exist positive constants $m_{i}, M_{i}, n_{i}$, and $N_{i}(i=1,2, \ldots, n)$ which are independent of the solutions of system (1), such that, for any positive solution $\left(x_{1}(k), x_{2}(k), \ldots, x_{n}(k), u_{1}(k), u_{2}(k), \ldots, u_{n}(k)\right)$ of system (1), one has

$$
\begin{gathered}
m_{i} \leq \liminf _{k \rightarrow+\infty} x_{i}(k) \leq \limsup _{k \rightarrow+\infty} x_{i}(k) \leq M_{i}, \\
n_{i} \leq \liminf _{k \rightarrow+\infty} u_{i}(k) \leq \limsup _{k \rightarrow+\infty} u_{i}(k) \leq N_{i}, \\
i=1,2, \ldots, n .
\end{gathered}
$$

Proof. Let $\left(x_{1}(k), x_{2}(k), \ldots, x_{n}(k), u_{1}(k), u_{2}(k), \ldots, u_{n}(k)\right)$ be any positive solution of system (1). From the first equation of system (1), it follows that

$$
x_{i}(k+1) \leq x_{i}(k) \exp \left\{a_{i}(k)+\sum_{j=1, j \neq i}^{n} c_{i j}(k)-b_{i}(k) x_{i}(k)\right\} .
$$

Thus, as a direct corollary of Lemma 5, according to (23), one has

$$
\limsup _{k \rightarrow+\infty}(k) \leq \frac{1}{b_{i}^{l}} \exp \left\{a_{i}^{u}+\sum_{j=1, j \neq i}^{n} c_{i j}^{u}-1\right\} \triangleq M_{i}>0 .
$$

For any small positive constant $\varepsilon>0$, from (24), it follows that there exists a positive constant $K_{1}>0$ such that, for all $k>K_{1}$ and $i=1,2, \ldots, n$,

$$
x_{i}(k) \leq M_{i}+\varepsilon
$$

For $k \geq K_{1}$, from (25) and system (1), we have

$$
u_{i}(k+1) \leq\left(1-f_{i}^{l}\right) u_{i}(k)+\sum_{j=1}^{n} g_{i j}(k)\left(M_{j}+\varepsilon\right) .
$$


Then, as a direct corollary of Lemma 7, according to (26), one has

$$
\limsup _{k \rightarrow+\infty} u_{i}(k) \leq \frac{1}{f_{i}^{l}} \sum_{j=1}^{n} g_{i j}^{u}\left(M_{j}+\varepsilon\right)
$$

Letting $\varepsilon \rightarrow 0$, it follows that

$$
\limsup _{k \rightarrow+\infty}(k) \leq \frac{1}{f_{i}^{l}} \sum_{j=1}^{n} g_{i j}^{u} M_{j} \triangleq N_{i}
$$

Thus, there exists a positive integer $K_{2}>K_{1}$, and we have, for $k>K_{2}$,

$$
u_{i}(k) \leq N_{i}+\varepsilon
$$

For $k \geq K_{2}$, from (29) and system (1), we have

$x_{i}(k+1) \geq x_{i}(k) \exp \left\{a_{i}(k)-b_{i}(k) x_{i}(k)-e_{i}(k)\left(N_{i}+\varepsilon\right)\right\}$.

Assuming that $a_{i}^{l}-e_{i}^{u} N_{i}>0$, for any $\varepsilon>0$, there exists a positive integer $K_{3}>K_{2}$ such that $a_{i}(k)-e_{i}(k)\left(N_{i}+\varepsilon\right)>0$ for $k>K_{3}$. Thus, as a direct corollary of Lemma 6 , according to $(30)$, one has

$$
\liminf _{k \rightarrow+\infty} x_{i}(k) \geq \min \left\{m_{i_{1} \varepsilon}, m_{i_{2} \varepsilon}\right\}
$$

where

$$
\begin{gathered}
m_{i_{1} \varepsilon}=\frac{a_{i}^{l}-e_{i}^{u}\left(N_{i}+\varepsilon\right)}{b_{i}^{u}}, \\
m_{i_{2} \varepsilon}=m_{i_{1} \varepsilon} \exp \left\{a_{i}^{l}-e_{i}^{u}\left(N_{i}+\varepsilon\right)-b_{i}^{u} M_{i}\right\} .
\end{gathered}
$$

Letting $\varepsilon \rightarrow 0$, it follows that

$$
\liminf _{k \rightarrow+\infty} x_{i}(k) \geq \frac{1}{2} \min \left\{m_{i_{1}}, m_{i_{2}}\right\} \triangleq m_{i}>0,
$$

where

$$
\begin{gathered}
m_{i_{1}}=\frac{a_{i}^{l}-e_{i}^{u} N_{i}}{b_{i}^{u}}, \\
m_{i_{2}}=m_{i_{1} \varepsilon} \exp \left\{a_{i}^{l}-e_{i}^{u} N_{i}-b_{i}^{u} M_{i}\right\} .
\end{gathered}
$$

From (33), for any $\varepsilon>0$, there exists a positive integer $K_{4}>K_{3}$ such that

$$
x_{i}(k) \geq m_{i}-\varepsilon
$$

for $k>K_{4}$.

From (35) and system (1), we have

$$
u_{i}(k+1) \geq\left(1-f_{i}^{u}\right) u_{i}(k)+\sum_{j=1}^{n} g_{i j}(k)\left(m_{j}-\varepsilon\right) \text {. }
$$

Then, as a direct corollary of Lemma 8, according to (36), one has

$$
\liminf _{k \rightarrow+\infty} u_{i}(k) \geq \frac{1}{f_{i}^{u}} \sum_{j=1}^{n} g_{i j}^{l}\left(m_{j}-\varepsilon\right) .
$$

Letting $\varepsilon \rightarrow 0$, it follows that

$$
\liminf _{k \rightarrow+\infty} u_{i}(k) \geq \frac{1}{f_{i}^{u}} \sum_{j=1}^{n} g_{i j}^{l} m_{j} \triangleq n_{i}>0 .
$$

Then, (24), (28), (33), and (38) show that system (1) is permanent. The proof is completed.

According to Theorem 9, we first prove that there is a bounded solution of system (1), and then construct a suitable Lyapunov function for system (1).

We denote by $\Omega$ the set of all solutions $\left(x_{1}(k), x_{2}(k)\right.$, $\left.\ldots, x_{n}(k), u_{1}(k), u_{2}(k), \ldots, u_{n}(k)\right)$ of system (1) satisfying $m_{i} \leq x_{i}(k) \leq M_{i}$ and $n_{i} \leq u_{i}(k) \leq N_{i}(i=1,2, \ldots, n)$ for all $k \in Z^{+}$.

Proposition 11. Assume that the conditions (2), (3), and (21) hold. Then $\Omega \neq \Phi$.

Proof. By the almost periodicity of $\left\{a_{i}(k)\right\},\left\{b_{i}(k)\right\},\left\{c_{i j}(k)\right\}$, $\left\{d_{i j}(k)\right\},\left\{e_{i}(k)\right\},\left\{f_{i}(k)\right\}$, and $\left\{g_{i j}(k)\right\}$, there exists an integer valued sequence $\left\{\delta_{p}\right\}$ with $\delta_{p} \rightarrow \infty$ as $p \rightarrow \infty$ such that

$$
\begin{gathered}
a_{i}\left(k+\delta_{p}\right) \longrightarrow a_{i}(k), \quad b_{i}\left(k+\delta_{p}\right) \longrightarrow b_{i}(k), \\
c_{i j}\left(k+\delta_{p}\right) \longrightarrow c_{i j}(k), \quad d_{i j}\left(k+\delta_{p}\right) \longrightarrow d_{i j}(k), \\
e_{i}\left(k+\delta_{p}\right) \longrightarrow e_{i}(k), \quad f_{i}\left(k+\delta_{p}\right) \longrightarrow f_{i}(k), \\
g_{i j}\left(k+\delta_{p}\right) \longrightarrow g_{i j}(k) \\
\text { as } p \longrightarrow \infty .
\end{gathered}
$$

Let $\varepsilon$ be an arbitrary small positive number. It follows from Theorem 10 that there exists a positive integer $N_{0}$ such that

$$
\begin{array}{r}
m_{i}-\varepsilon \leq x_{i}(k) \leq M_{i}+\varepsilon, \quad n_{i}-\varepsilon \leq u_{i}(k) \leq N_{i}+\varepsilon \\
k>N_{0} .
\end{array}
$$

Write $x_{i p}(k)=x_{i}\left(k+\delta_{p}\right)$ and $u_{i p}(k)=u_{i}\left(k+\delta_{p}\right)$ for $k \geq$ $N_{0}-\delta_{p}$ and $p=1,2, \ldots$. For any positive integer $q$, it is easy to see that there exists a sequence $\left\{x_{i p}(k): p \geq q\right\}$ such that the sequence $x_{p}(k)$ has a subsequence, denoted by $\left\{x_{i p}(k)\right\}$ again, converging on any finite interval of $Z^{+}$as $p \rightarrow \infty$. Thus we have a sequence $\left\{y_{i}(k)\right\}$ such that

$$
\begin{array}{r}
x_{i p}(k) \longrightarrow y_{i}(k), \quad u_{i p}(k) \longrightarrow v_{i}(k) \\
\text { for } k \in Z^{+} \text {as } p \longrightarrow \infty .
\end{array}
$$


This, combined with

$$
\begin{gathered}
x_{i p}(k+1) \\
=x_{i p}(k) \exp \left\{a_{i}\left(k+\delta_{p}\right)-b_{i}\left(k+\delta_{p}\right) x_{i p}(k)\right. \\
+\sum_{j=1, j \neq i}^{n} c_{i j}\left(k+\delta_{p}\right) \\
\times \frac{x_{j p}(k)}{d_{i j}\left(k+\delta_{p}\right)+x_{j p}(k)} \\
\left.-e_{i}\left(k+\delta_{p}\right) u_{i p}(k)\right\}, \\
u_{i p}(k+1)=\left(1-f_{i}\left(k+\delta_{p}\right)\right) u_{i p}(k) \\
+\sum_{j=1}^{n} g_{i j}\left(k+\delta_{p}\right) x_{j p}(k), \quad i=1,2, \ldots, n,
\end{gathered}
$$

gives us

$$
\begin{aligned}
y_{i}(k+1)=y_{i}(k) \exp \left\{a_{i}(k)-b_{i}(k) y_{i}(k)\right. & \\
& +\sum_{j=1, j \neq i}^{n} c_{i j}(k) \frac{y_{j}(k)}{d_{i j}(k)+y_{j}(k)} \\
& \left.-e_{i}(k) v_{i}(k)\right\}, \\
v_{i}(k+1)=\left(1-f_{i}(k)\right) v_{i}(k) & \\
+ & +\sum_{j=1}^{n} g_{i j}(k) y_{j}(k), \quad i=1,2, \ldots, n .
\end{aligned}
$$

We can easily see that $\left(y_{1}(k), y_{2}(k), \ldots, y_{n}(k), v_{1}(k), v_{2}(k), \ldots\right.$, $\left.v_{n}(k)\right)$ is a solution of system (1) and $m_{i}-\varepsilon \leq y_{i}(k) \leq M_{i}+\varepsilon$ and $n_{i}-\varepsilon \leq v_{i}(k) \leq N_{i}+\varepsilon$ for $k \in Z^{+}$. Since $\varepsilon$ is an arbitrary small positive number, it follows that $m_{i} \leq y_{i}(k) \leq M_{i}$ and $n_{i} \leq v_{i}(k) \leq N_{i}$ and hence we complete the proof.

\section{Stability of Almost Periodic Solution}

In this section, by constructing a nonnegative Lyapunov function, we will obtain sufficient conditions for uniform asymptotical stability of positive almost periodic solution of system (1).
Theorem 12. Assume that the conditions (2), (3), and (21) hold; moreover, $0<\beta<1$, where

$$
\begin{aligned}
& \beta=\min \left\{\min _{1 \leq i \leq n} \beta_{i}^{1}, \min _{1 \leq i \leq n} \beta_{i}^{2}\right\}, \\
& \beta_{i}^{1}=2 b_{i}^{l} m_{i}-b_{i}^{u 2} M_{i}^{2}-\sum_{j=1}^{n} g_{j i}^{u 2} M_{i}^{2} \\
& -\sum_{j=1, j \neq i}^{n} c_{j i}^{u 2}-\left(1+2 b_{i}^{u} M_{i}\right) c_{i j}^{u} \\
& -\frac{1}{2} \sum_{l=1, l \neq i, j}^{n} c_{l i}^{u} c_{l j}^{u}-\frac{1}{2} \sum_{l=1}^{n} g_{l i}^{u} g_{l j}^{u} M_{i} M_{j} \\
& -e_{i}^{u}\left(b_{i}^{u} M_{i}+1\right)-g_{i i}^{u} M_{i}\left(1-f_{i}^{l}\right) \\
& -\sum_{j=1, j \neq i}^{n}\left[\left(1+2 b_{j}^{u} M_{j}\right) c_{j i}^{u}+\frac{1}{2} \sum_{l=1, l \neq i, j}^{n} c_{l i}^{u} c_{l j}^{u}\right. \\
& +\frac{1}{2} \sum_{l=1}^{n} g_{l i}^{u} g_{l j}^{u} M_{i} M_{j}+\left(1-f_{j}^{l}\right) g_{j i}^{u} M_{i} \\
& \left.+e_{j}^{u} c_{j i}^{u}\right] \\
& \beta_{i}^{2}=f_{i}^{l}\left(2-f_{i}^{u}\right)-e_{i}^{u}\left(b_{i}^{u} M_{i}+1\right)-g_{i i}^{u} M_{i}\left(1-f_{i}^{l}\right) \\
& -e_{i}^{u 2}-\sum_{j=1, j \neq i}^{n}\left[\left(1-f_{i}^{l}\right) g_{i j}^{u} M_{j}+e_{i}^{u} c_{i j}^{u}\right],
\end{aligned}
$$

$i=1,2, \ldots, n$. Then there exists a unique uniformly asymptotically stable almost periodic solution $\left(x_{1}(k), x_{2}(k), \ldots, x_{n}(k)\right.$, $\left.u_{1}(k), u_{2}(k), \ldots, u_{n}(k)\right)$ of system (1) which is bounded by $\Omega$ for all $k \in N^{+}$.

Proof. Let $p_{i}(k)=\ln x_{i}(k), i=1,2, \ldots, n$. From system (1), we have

$$
\begin{gathered}
p_{i}(k+1)=p_{i}(k)+a_{i}(k)-b_{i}(k) e^{p_{i}(k)} \\
+\sum_{j=1, j \neq i}^{n} c_{i j}(k) \frac{e^{p_{j}(k)}}{d_{i j}(k)+e^{p_{j}(k)}}-e_{i}(k) u_{i}(k), \\
\Delta u_{i}(k)=-f_{i}(k) u_{i}(k)+\sum_{j=1}^{n} g_{i j}(k) e^{p_{j}(k)}, \\
i=1,2, \ldots, n .
\end{gathered}
$$

From Proposition 11, we know that system (45) has a bounded solution $\left(p_{1}(k), p_{2}(k), \ldots, p_{n}(k), u_{1}(k), u_{2}(k), \ldots, u_{n}(k)\right)$ satisfying

$$
\begin{array}{r}
\ln m_{i} \leq p_{i}(k) \leq \ln M_{i}, \quad n_{i} \leq u_{i}(k) \leq N_{i}, \\
i=1,2, \ldots, n, \quad k \in Z^{+} .
\end{array}
$$


Hence, $\left|p_{i}(k)\right| \leq A_{i}$ and $\left|u_{i}(k)\right| \leq B_{i}$, where $A_{i}=$ $\max \left\{\left|\ln m_{i}\right|,\left|\ln M_{i}\right|\right\}$ and $B_{i}=\max \left\{n_{i}, N_{i}\right\}, i=1,2, \ldots, n$.

For $(X, U) \in R^{n+n}$, we define the norm $\|(X, U)\|=$ $\sum_{i=1}^{n}\left|x_{i}\right|+\sum_{i=1}^{n}\left|u_{i}\right|$.

Consider that the product system of (45) is

$$
\begin{aligned}
p_{i}(k+1)= & p_{i}(k)+a_{i}(k)-b_{i}(k) e^{p_{i}(k)} \\
& +\sum_{j=1, j \neq i}^{n} c_{i j}(k) \frac{e^{p_{j}(k)}}{d_{i j}(k)+e^{p_{j}(k)}}-e_{i}(k) u_{i}(k), \\
\Delta u_{i}(k)=-f_{i}(k) u_{i}(k)+\sum_{j=1}^{n} g_{i j}(k) e^{p_{j}(k)}, & \\
q_{i}(k+1)= & q_{i}(k)+a_{i}(k)-b_{i}(k) e^{q_{i}(k)} \\
& +\sum_{j=1, j \neq i}^{n} c_{i j}(k) \frac{e^{q_{j}(k)}}{d_{i j}(k)+e^{q_{j}(k)}}-e_{i}(k) w_{i}(k), \\
\Delta w_{i}(k)=- & f_{i}(k) w_{i}(k)+\sum_{j=1}^{n} g_{i j}(k) e^{q_{j}(k)}, \quad i=1,2, \ldots, n .
\end{aligned}
$$

We assume that $Q=\left(p_{1}(k), p_{2}(k), \ldots, p_{n}(k), u_{1}(k), u_{2}(k), \ldots\right.$, $\left.u_{n}(k)\right)$ and $W=\left(q_{1}(k), q_{2}(k), \ldots, q_{n}(k), w_{1}(k), w_{2}(k), \ldots\right.$, $\left.w_{n}(k)\right)$ are any two solutions of system (45) defined on $Z^{+} \times S^{*}$; then, $\|Q\| \leq B$ and $\|W\| \leq B$, where $B=\sum_{i=1}^{n}\left\{A_{i}+B_{i}\right\}$ and $S^{*}=\left\{\left(p_{1}(k), p_{2}(k), \ldots, p_{n}(k), u_{1}(k)\right.\right.$, $\left.u_{2}(k), \ldots, u_{n}(k)\right) \mid \ln m_{i} \leq p_{i}(n) \leq \ln M_{i}, n_{i} \leq u_{i}(k) \leq N_{i}$, $\left.i=1,2, \ldots, n, k \in Z^{+}\right\}$.

Let us construct a Lyapunov function defined on $Z^{+} \times S^{*} \times$ $S^{*}$ as follows:

$$
V(k, Q, W)=\sum_{i=1}^{n}\left\{\left(p_{i}(k)-q_{i}(k)\right)^{2}+\left(u_{i}(k)-w_{i}(k)\right)^{2}\right\} .
$$

It is obvious that the norm $\|Q-W\|=\sum_{i=1}^{n}\left\{\left|p_{i}(k)-q_{i}(k)\right|+\right.$ $\left.\left|u_{i}(k)-w_{i}(k)\right|\right\}$ is equivalent to $\|Q-W\|_{*}=\left[\sum_{i=1}^{n}\left\{\left(p_{i}(k)-\right.\right.\right.$ $\left.\left.\left.q_{i}(k)\right)^{2}+\left(u_{i}(k)-w_{i}(k)\right)^{2}\right\}\right]^{1 / 2}$; that is, there are two constants $c_{1}>0$ and $c_{2}>0$, such that

$$
c_{1}\|Q-W\| \leq\|Q-W\|_{*} \leq c_{2}\|Q-W\|,
$$

and then,

$$
\left(c_{1}\|Q-W\|\right)^{2} \leq V(k, Q, W) \leq\left(c_{2}\|Q-W\|\right)^{2} .
$$

Let $\psi, \varphi \in C\left(R^{+}, R^{+}\right), \psi(x)=c_{1}^{2} x^{2}, \varphi(x)=c_{2}^{2} x^{2}$; then, condition (i) of Theorem 9 is satisfied. have

Moreover, for any $(k, Q, W),(k, \bar{Q}, \bar{W}) \in Z^{+} \times S^{*} \times S^{*}$, we

$$
\begin{aligned}
& |V(k, Q, W)-V(k, \bar{Q}, \bar{W})| \\
& =\mid \sum_{i=1}^{n}\left\{\left(p_{i}(k)-q_{i}(k)\right)^{2}+\left(u_{i}(k)-w_{i}(k)\right)^{2}\right\}
\end{aligned}
$$

$$
\begin{aligned}
& -\sum_{i=1}^{n}\left\{\left(\overline{p_{i}}(k)-\overline{q_{i}}(k)\right)^{2}+\left(\overline{u_{i}}(k)-\overline{w_{i}}(k)\right)^{2}\right\} \mid \\
& \leq \sum_{i=1}^{n}\left|\left(p_{i}(k)-q_{i}(k)\right)^{2}-\left(\overline{p_{i}}(k)-\overline{q_{i}}(k)\right)^{2}\right| \\
& +\sum_{i=1}^{n}\left|\left(u_{i}(k)-w_{i}(k)\right)^{2}-\left(\overline{u_{i}}(k)-\overline{w_{i}}(k)\right)^{2}\right| \\
& =\sum_{i=1}^{n}\left|\left(p_{i}(k)-q_{i}(k)\right)+\left(\overline{p_{i}}(k)-\overline{q_{i}}(k)\right)\right| \\
& \times\left|\left(p_{i}(k)-q_{i}(k)\right)-\left(\overline{p_{i}}(k)-\overline{q_{i}}(k)\right)\right| \\
& +\sum_{i=1}^{n}\left|\left(u_{i}(k)-w_{i}(k)\right)+\left(\overline{u_{i}}(k)-\overline{w_{i}}(k)\right)\right| \\
& \times\left|\left(u_{i}(k)-w_{i}(k)\right)-\left(\overline{u_{i}}(k)-\overline{w_{i}}(k)\right)\right| \\
& \leq \sum_{i=1}^{n}\left(\left|p_{i}(k)\right|+\left|q_{i}(k)\right|+\left|\overline{p_{i}}(k)\right|+\left|\overline{q_{i}}(k)\right|\right) \\
& \times\left(\left|p_{i}(k)-\overline{p_{i}}(k)\right|+\left|q_{i}(k)-\overline{q_{i}}(k)\right|\right) \\
& +\sum_{i=1}^{n}\left(\left|u_{i}(k)\right|+\left|w_{i}(k)\right|+\left|\overline{u_{i}}(k)\right|+\left|\overline{w_{i}}(k)\right|\right) \\
& \times\left(\left|u_{i}(k)-\overline{u_{i}}(k)\right|+\left|w_{i}(k)-\overline{w_{i}}(k)\right|\right) \\
& \leq L\left[\sum_{i=1}^{n}\left\{\left|p_{i}(k)-\overline{p_{i}}(k)\right|+\left|u_{i}(k)-\overline{u_{i}}(k)\right|\right\}\right. \\
& \left.+\sum_{i=1}^{n}\left\{\left|q_{i}(k)-\overline{q_{i}}(k)\right|+\left|w_{i}(k)-\overline{w_{i}}(k)\right|\right\}\right] \\
& =L(\|Q-\bar{Q}\|+\|W-\bar{W}\|),
\end{aligned}
$$

where $\bar{Q}=\left(\overline{p_{1}}(k), \overline{p_{2}}(k), \ldots, \overline{p_{n}}(k), \overline{u_{1}}(k), \overline{u_{2}}(k), \ldots, \overline{u_{n}}(k)\right)$, $\bar{W}=\left(\overline{q_{1}}(k), \overline{q_{2}}(k), \ldots, \overline{q_{n}}(k), \overline{w_{1}}(k), \overline{w_{2}}(k), \ldots, \overline{w_{n}}(k)\right)$, and $L=4 \max \left\{\max _{1 \leq i \leq n}\left\{A_{i}\right\}, \max _{1 \leq i \leq n}\left\{B_{i}\right\}\right\}$. Thus, condition (ii) of Theorem 9 is satisfied.

Finally, calculating the $\Delta V(k)$ of $V(k)$ along the solutions of system (47), we have

$$
\begin{aligned}
\Delta V_{(47)}(k) \\
=V(k+1)-V(k) \\
=\sum_{i=1}^{n}\left\{\left(p_{i}(k+1)-q_{i}(k+1)\right)^{2}+\left(u_{i}(k+1)-w_{i}(k+1)\right)^{2}\right\} \\
\quad-\sum_{i=1}^{n}\left\{\left(p_{i}(k)-q_{i}(k)\right)^{2}+\left(u_{i}(k)-w_{i}(k)\right)^{2}\right\} \\
=\sum_{i=1}^{n}\left\{\left(p_{i}(k+1)-q_{i}(k+1)\right)^{2}-\left(p_{i}(k)-q_{i}(k)\right)^{2}\right.
\end{aligned}
$$




$$
\begin{aligned}
& \left.+\left(u_{i}(k+1)-w_{i}(k+1)\right)^{2}-\left(u_{i}(k)-w_{i}(k)\right)^{2}\right\}
\end{aligned}
$$

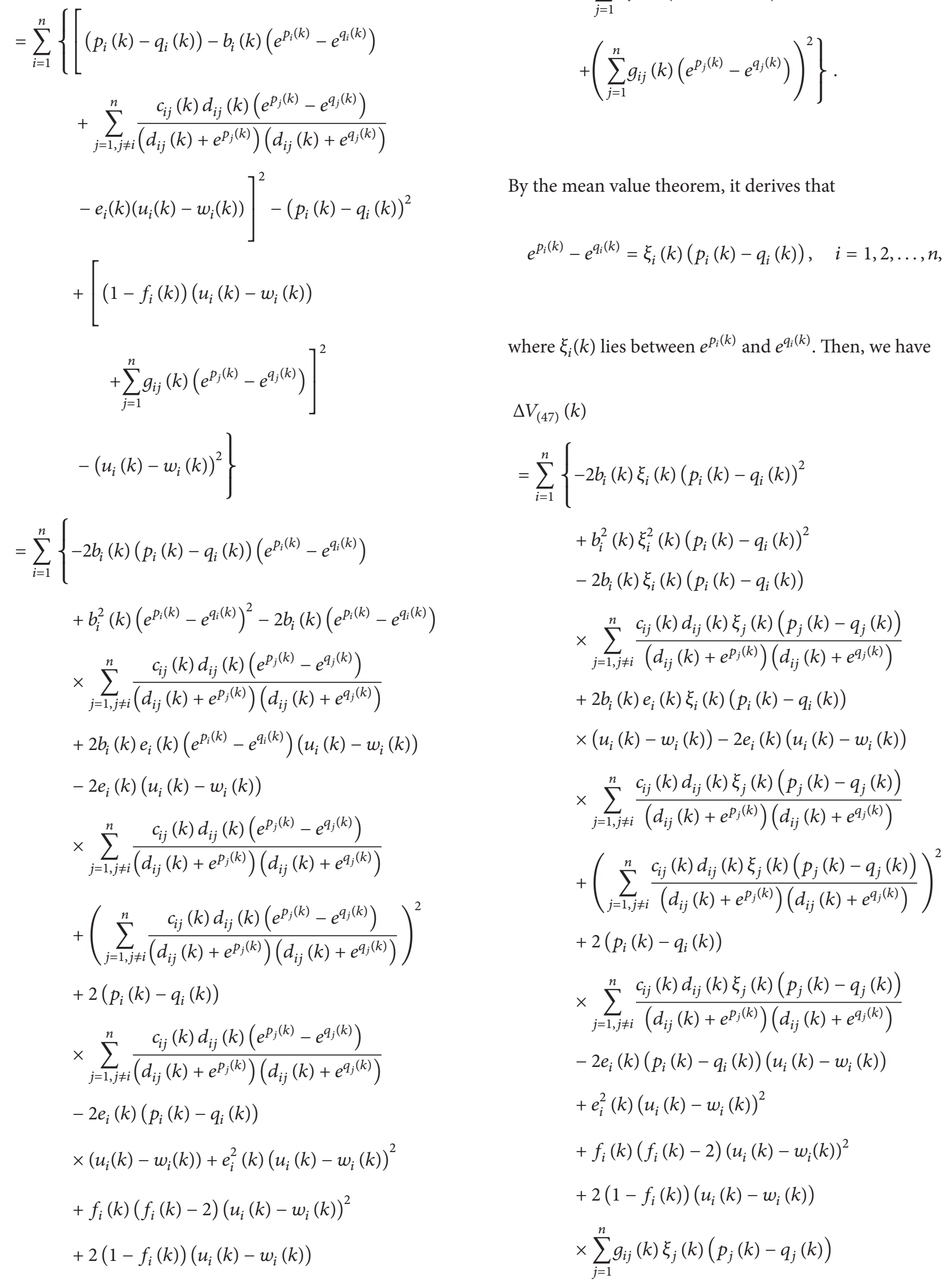

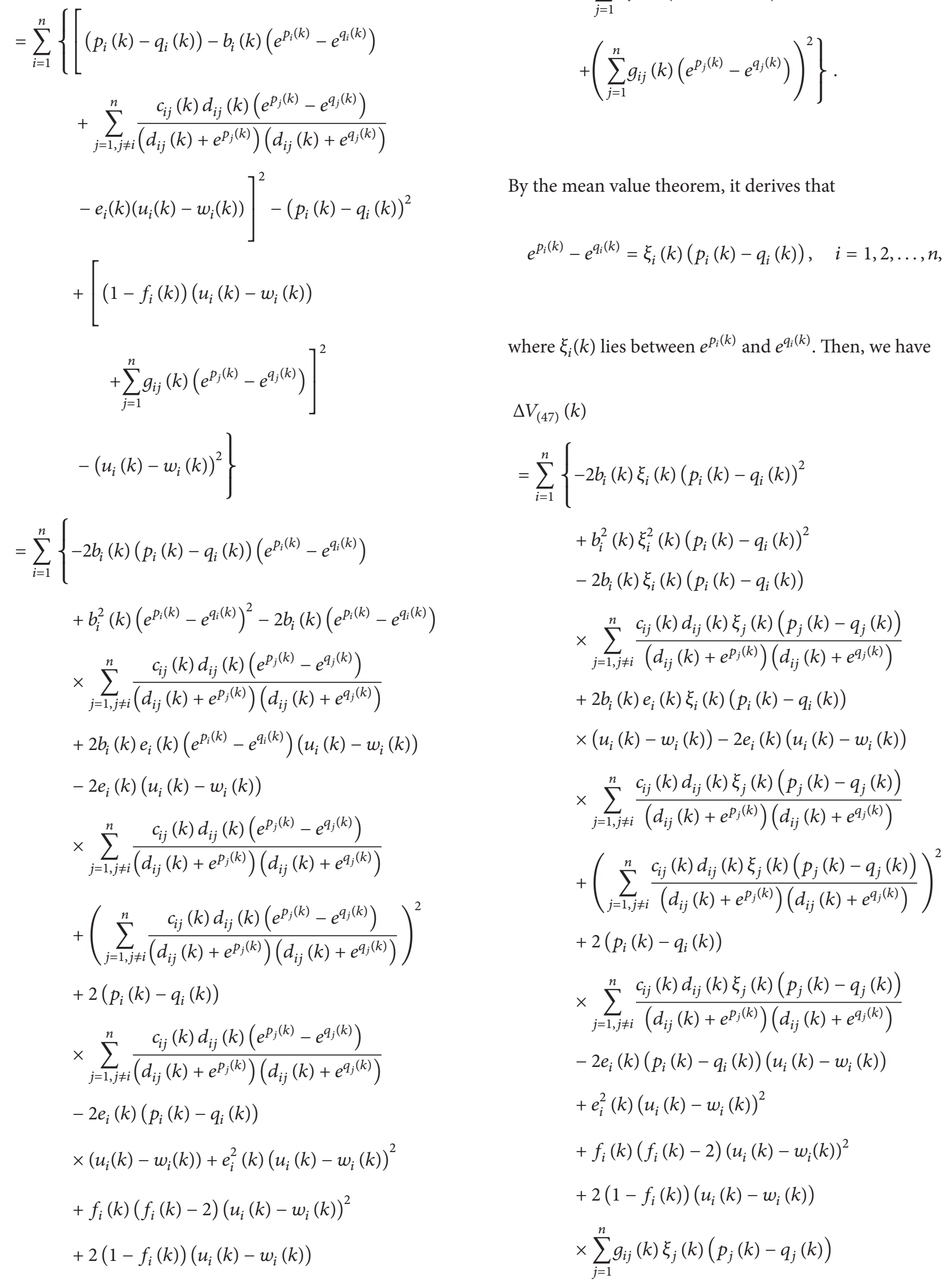

$$
\begin{aligned}
& \times \sum_{j=1}^{n} g_{i j}(k)\left(e^{p_{j}(k)}-e^{q_{j}(k)}\right)
\end{aligned}
$$

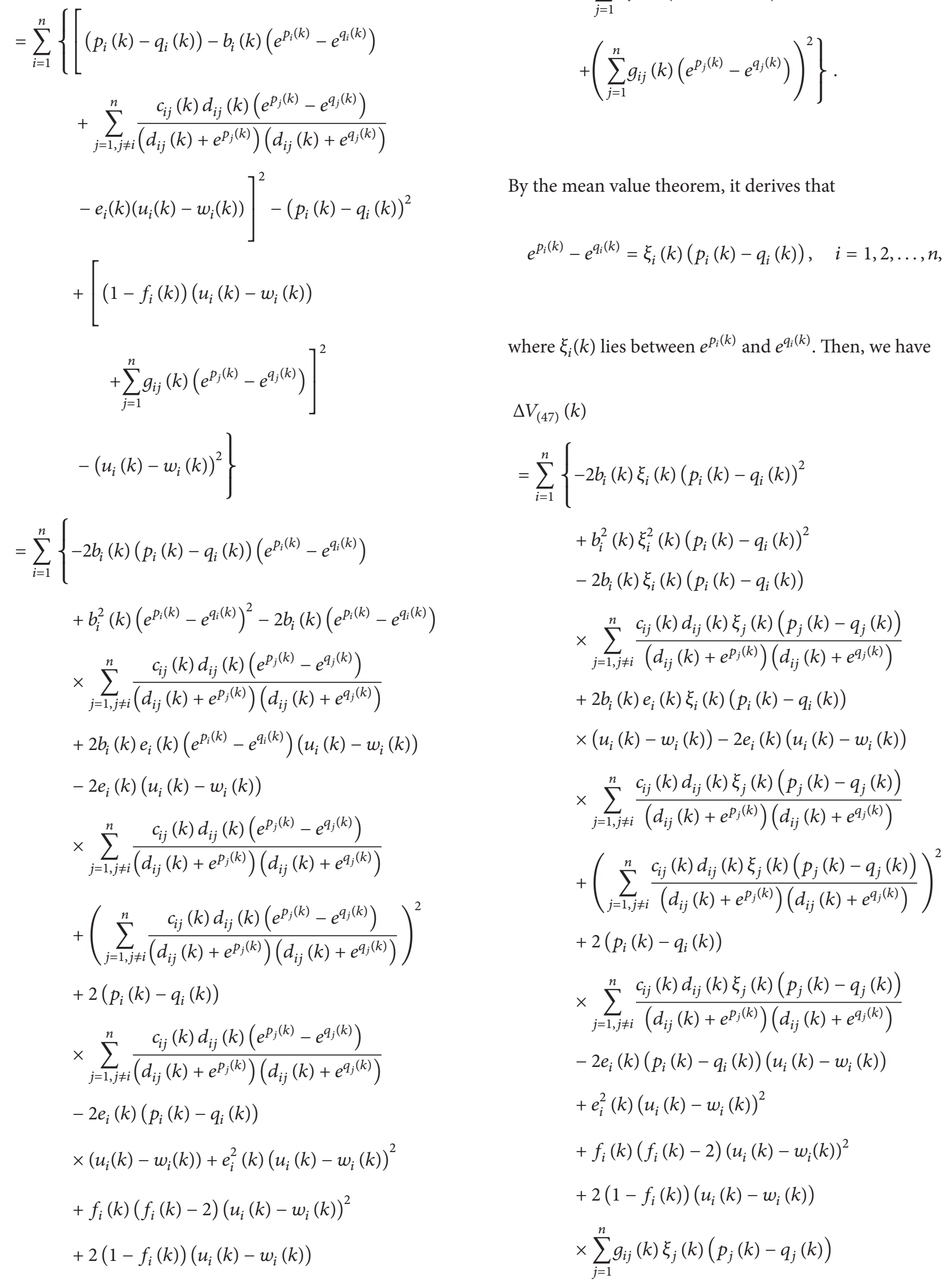

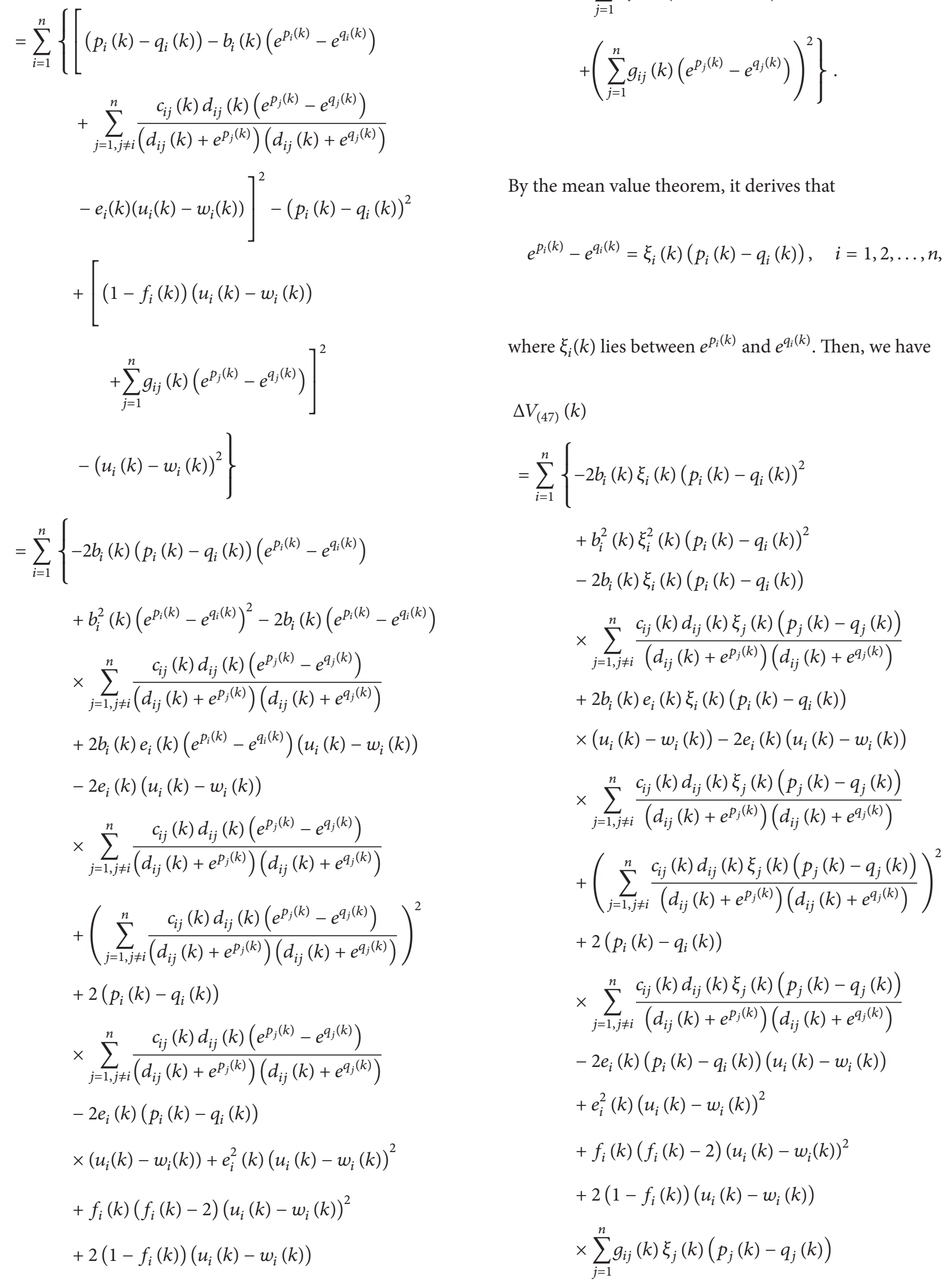

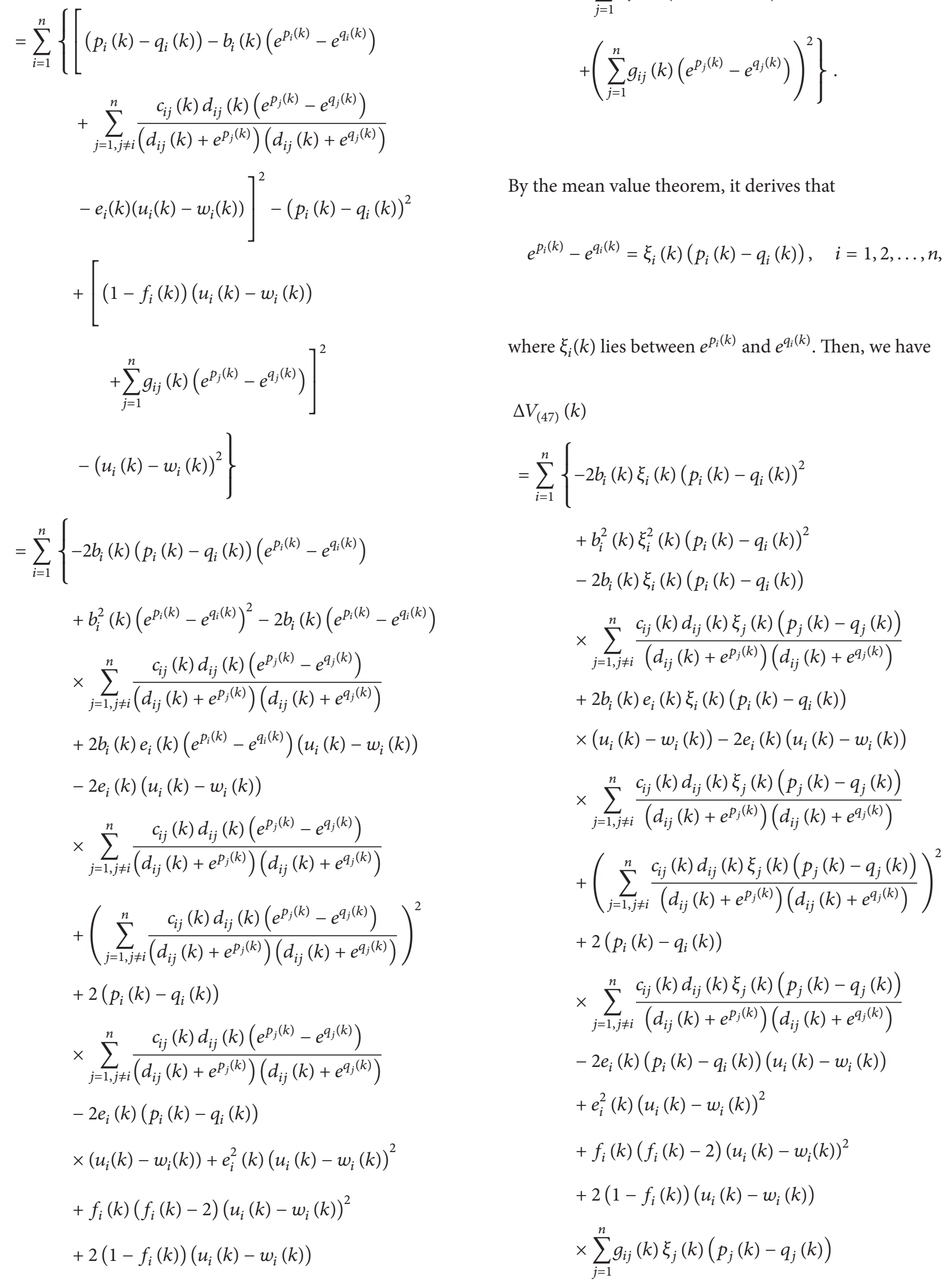

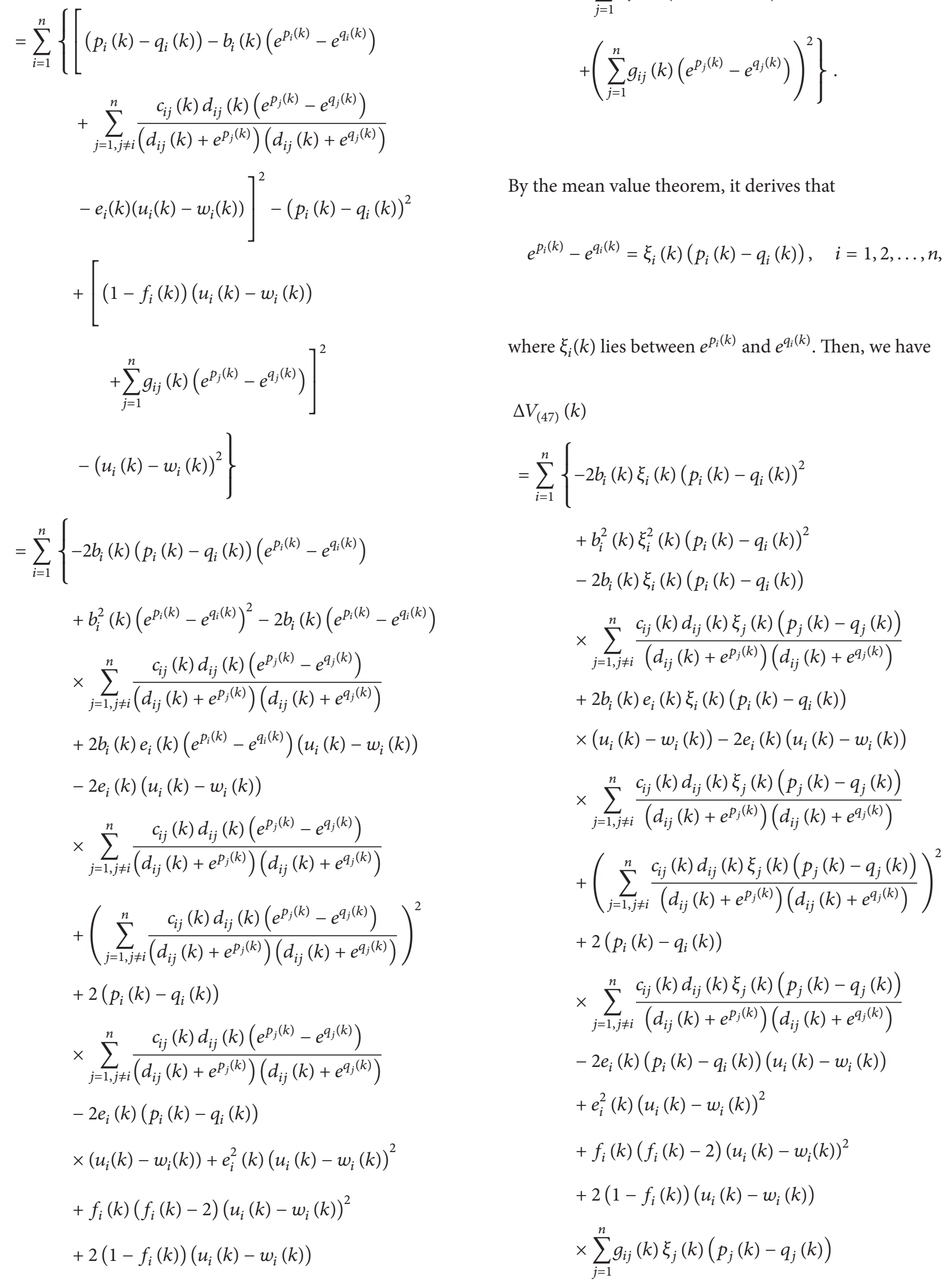

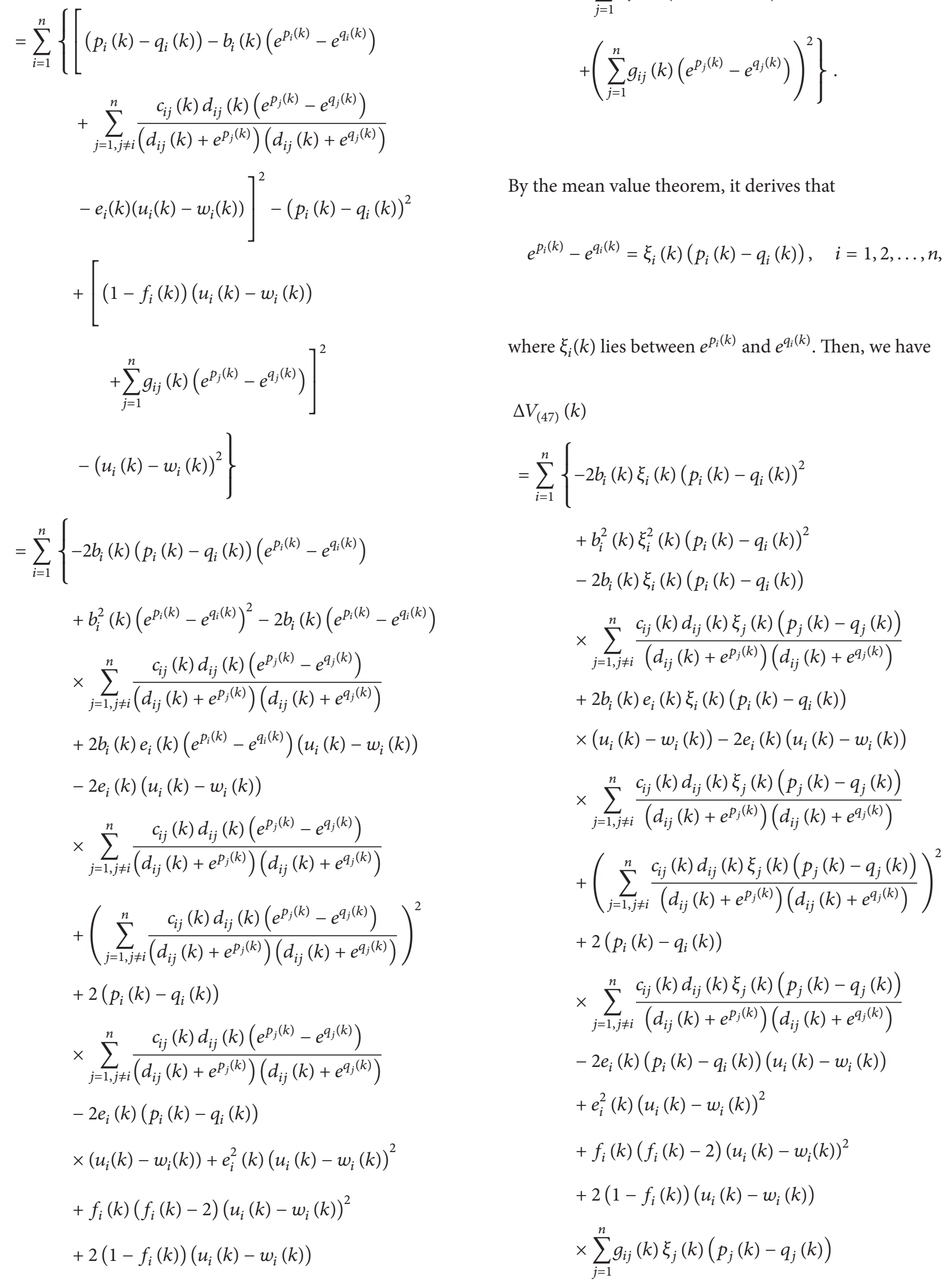

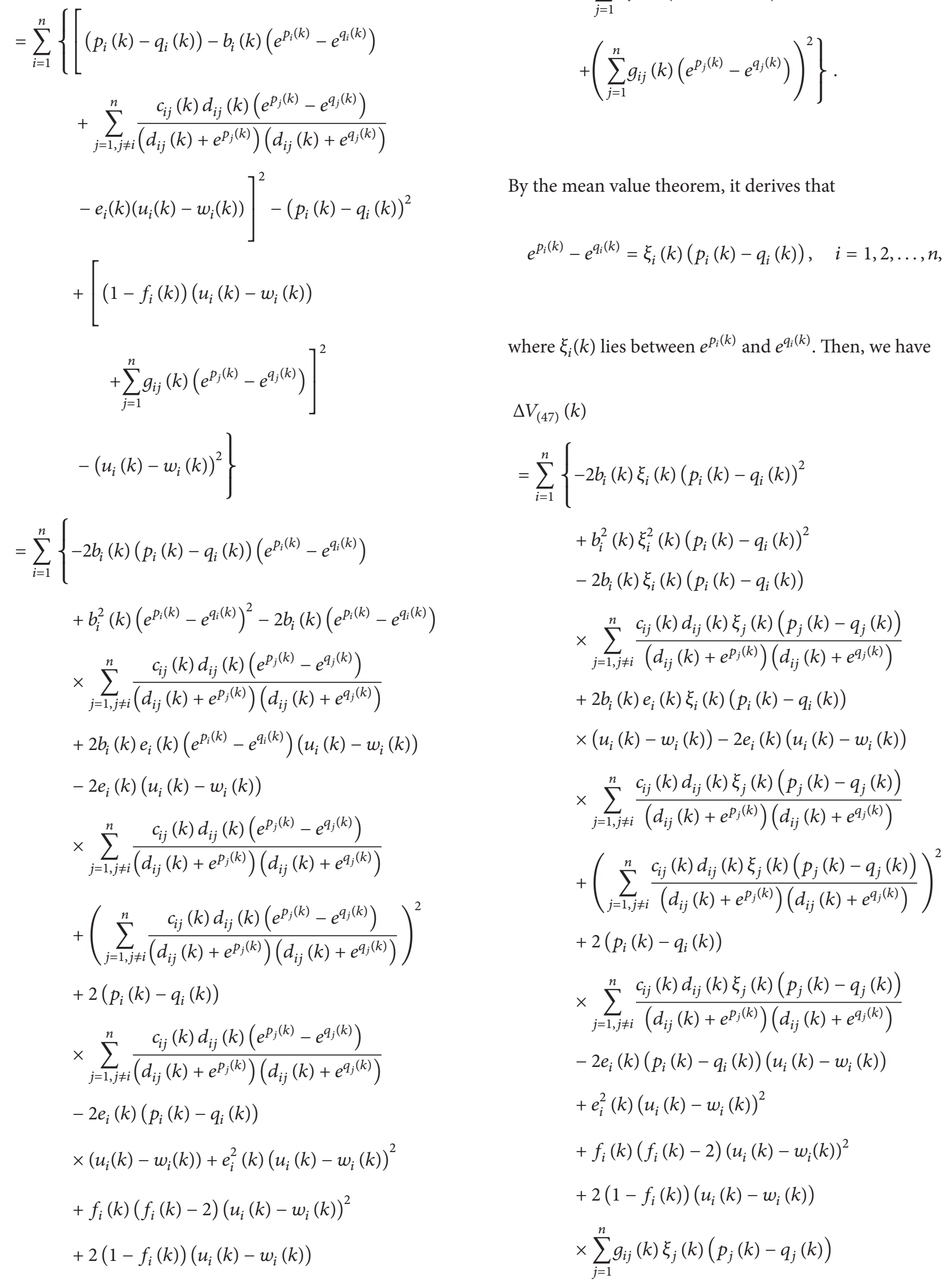

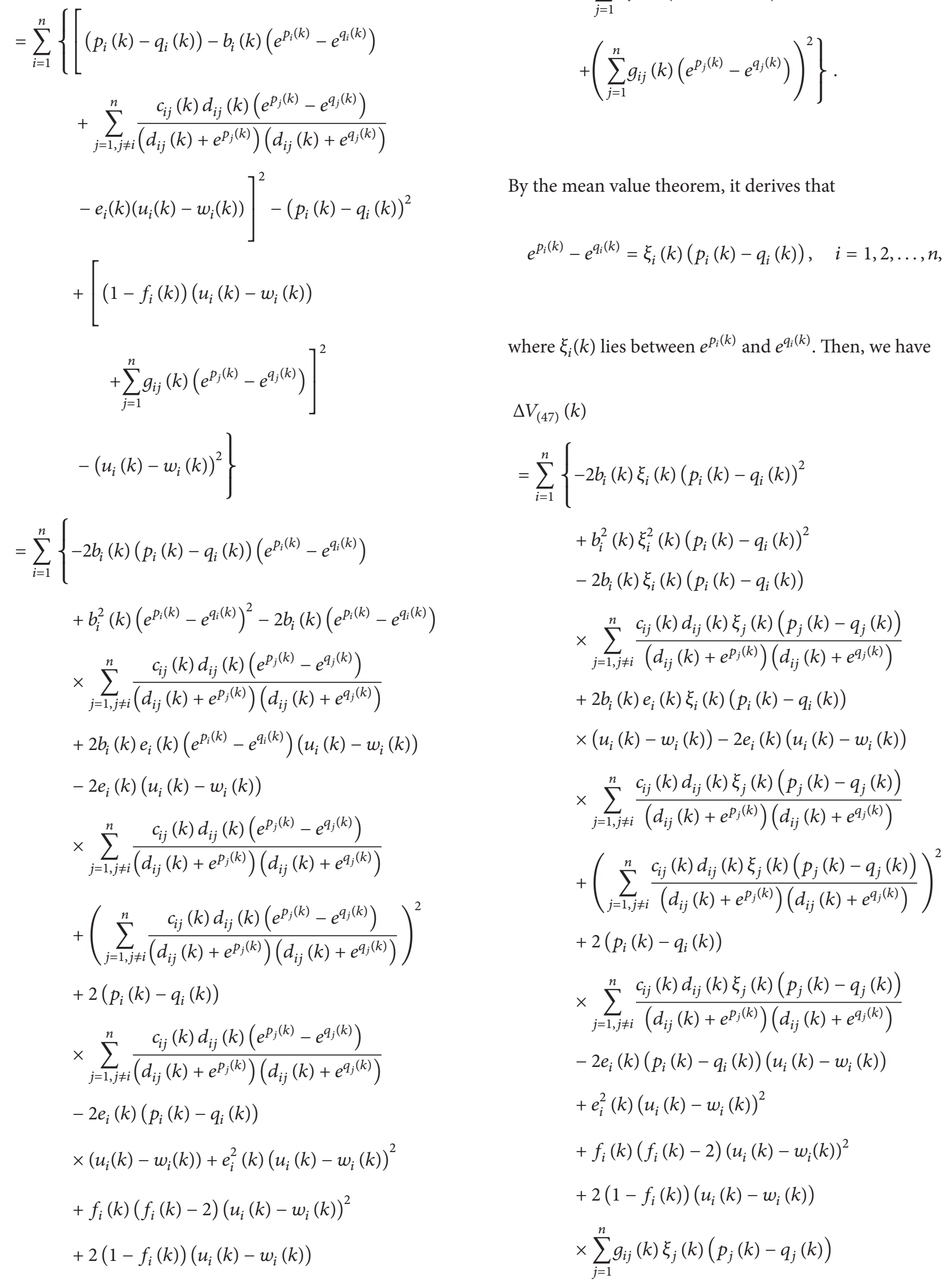

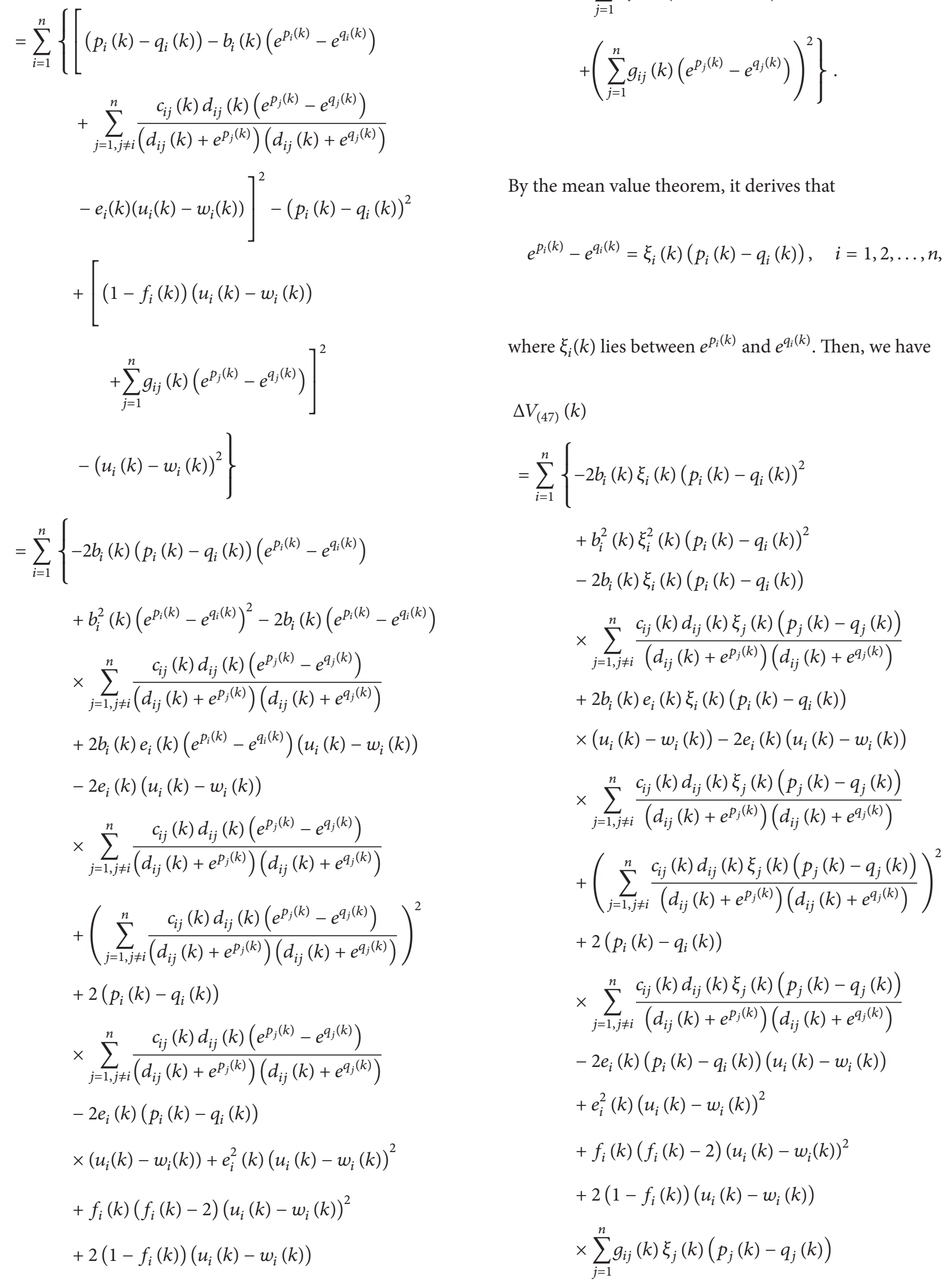

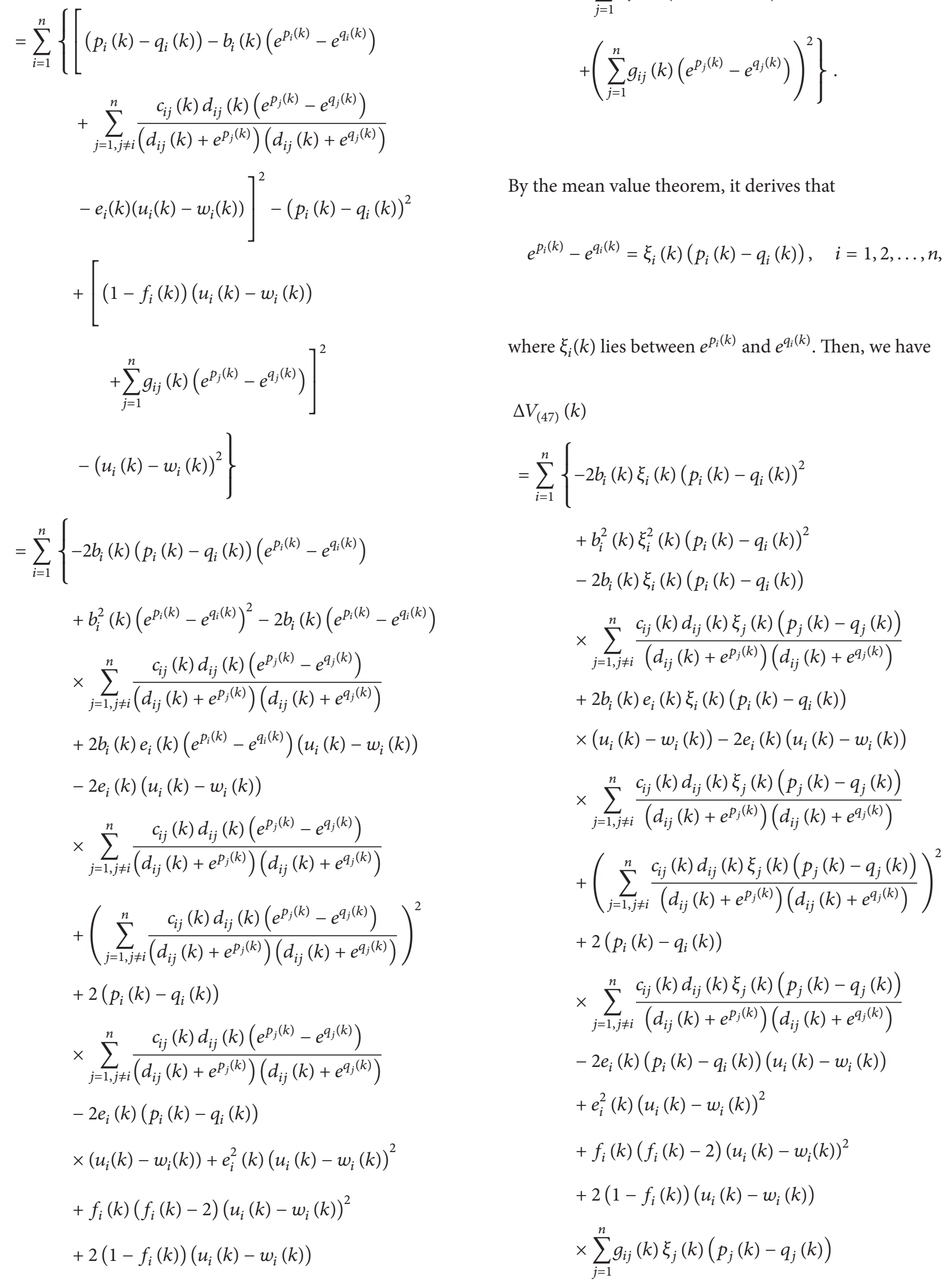

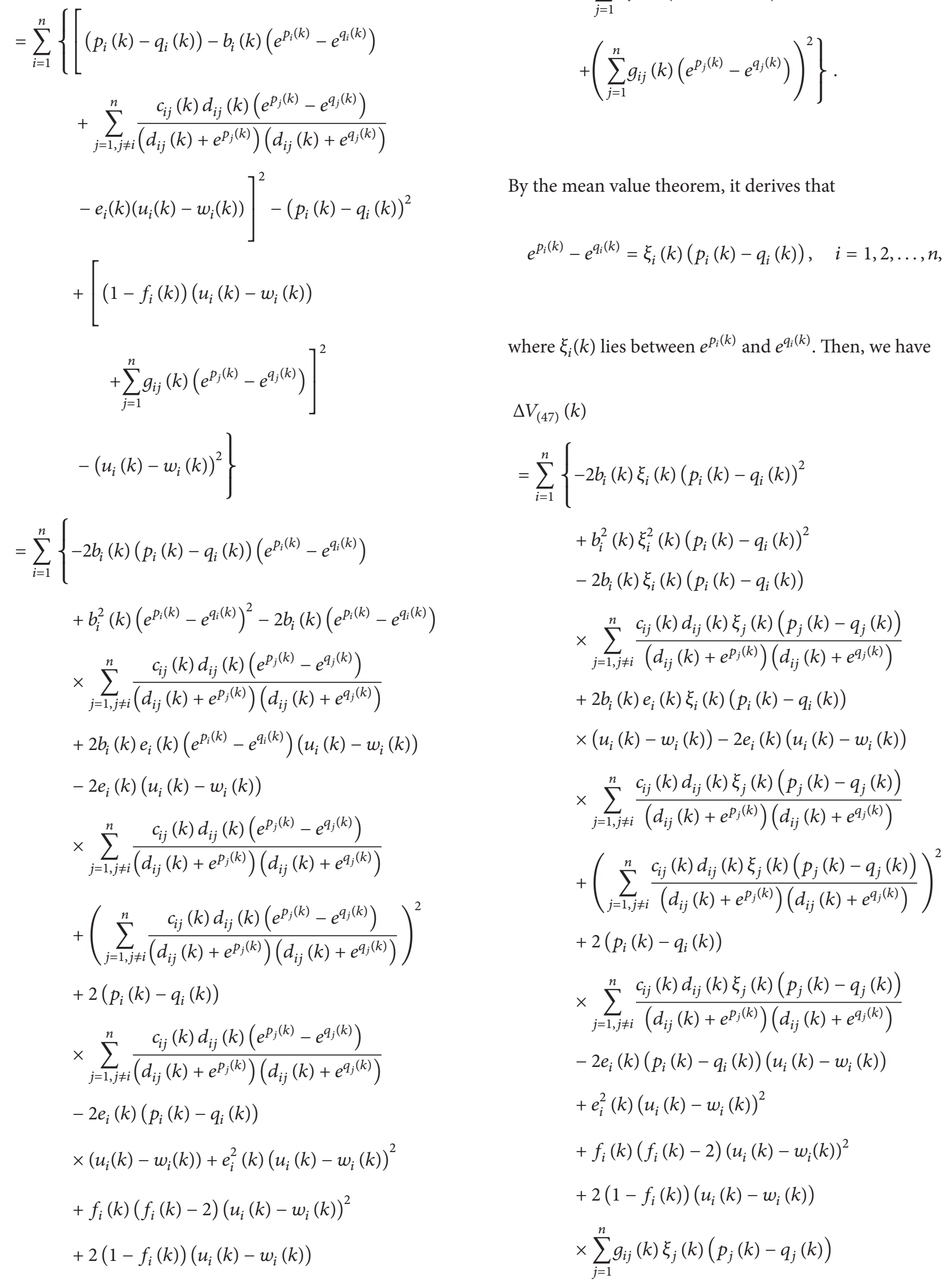

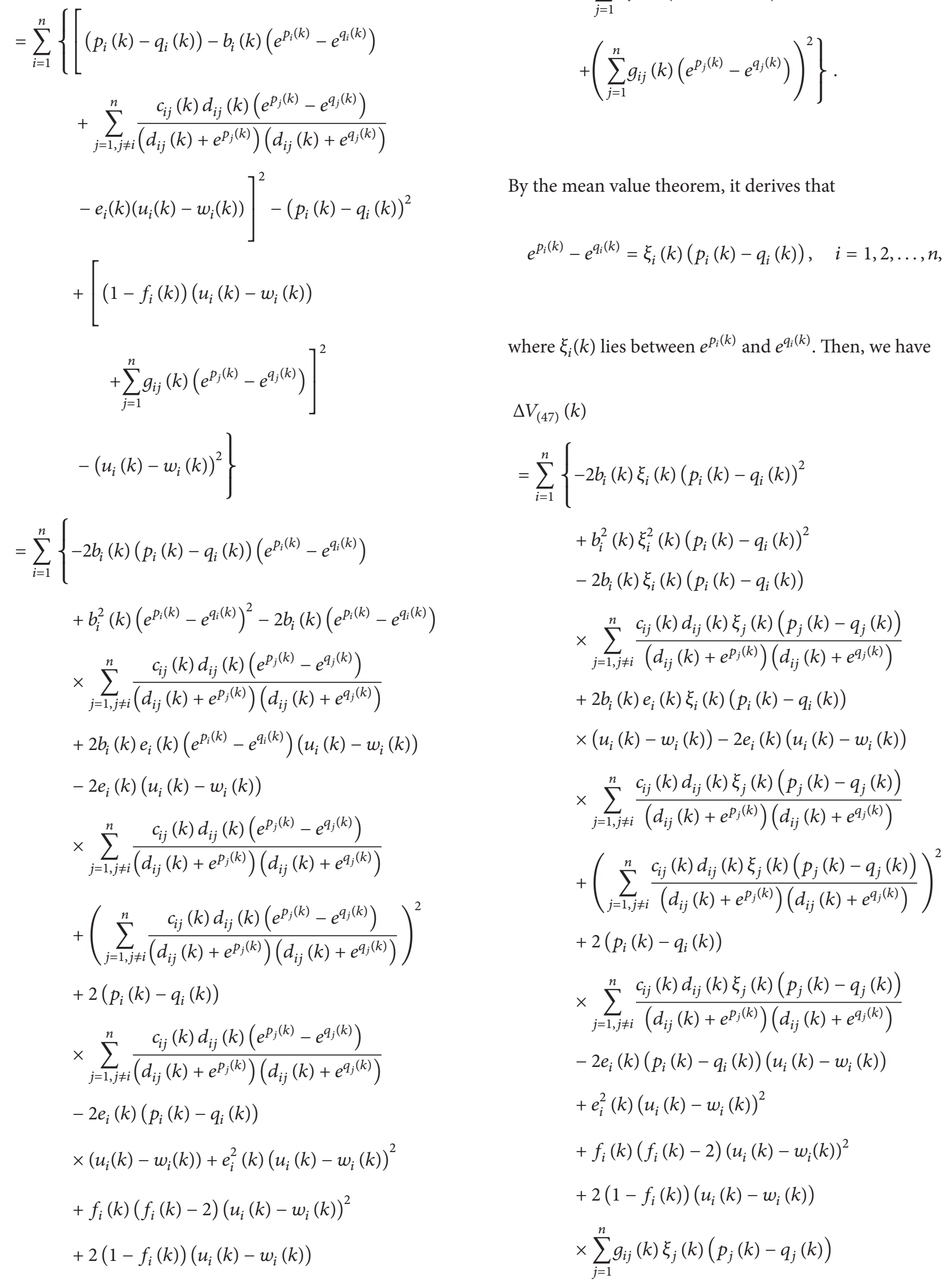

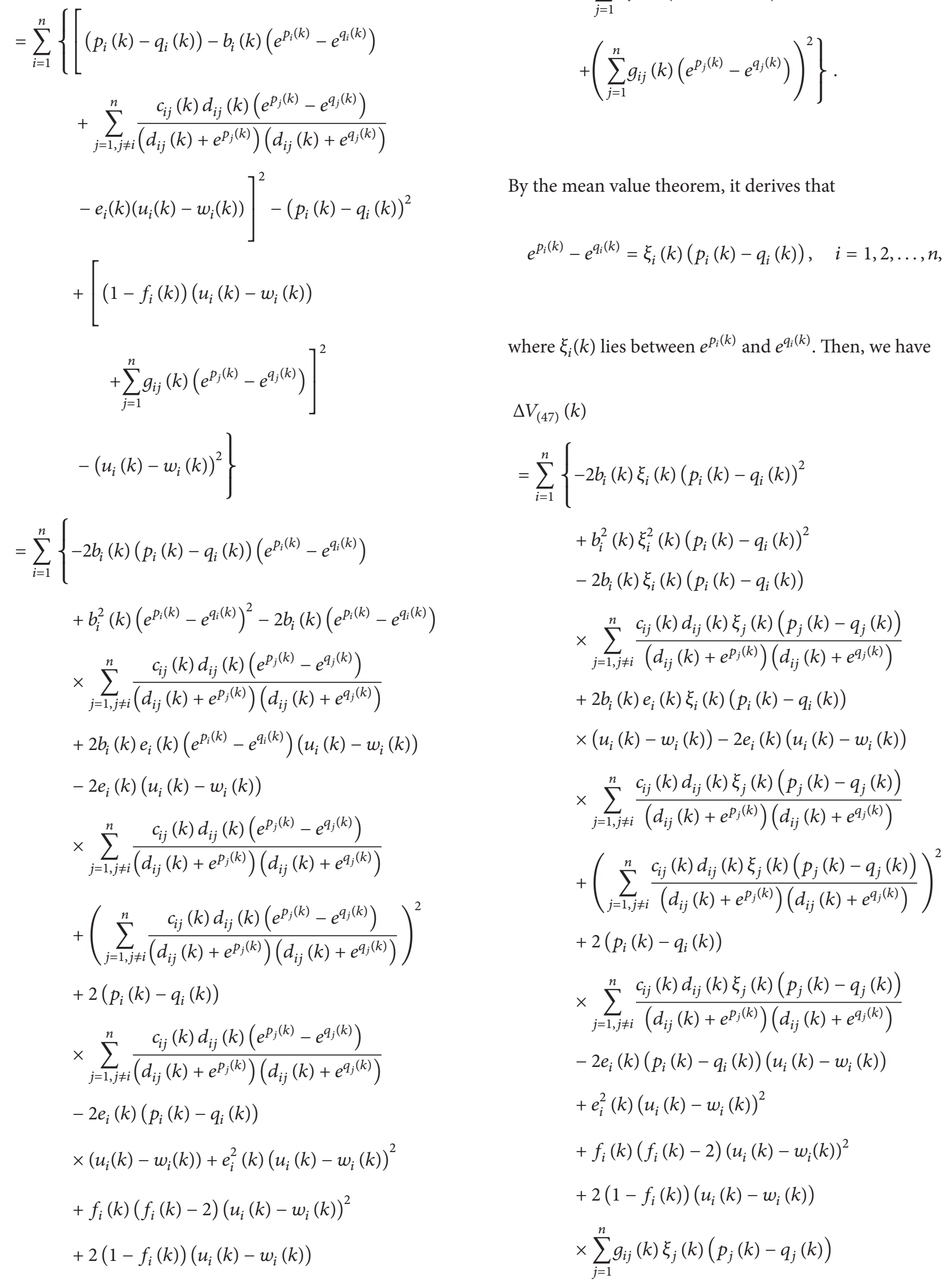

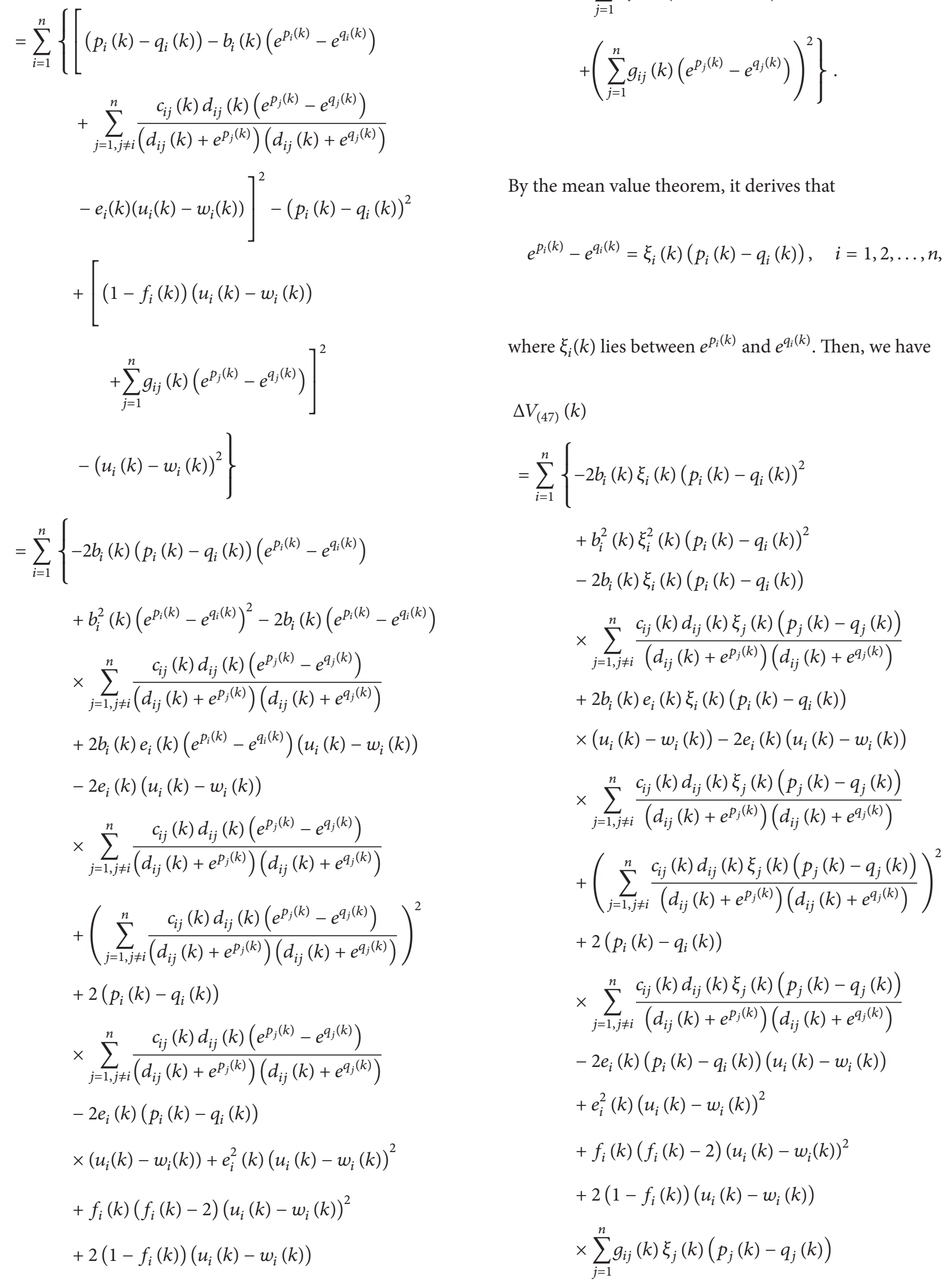

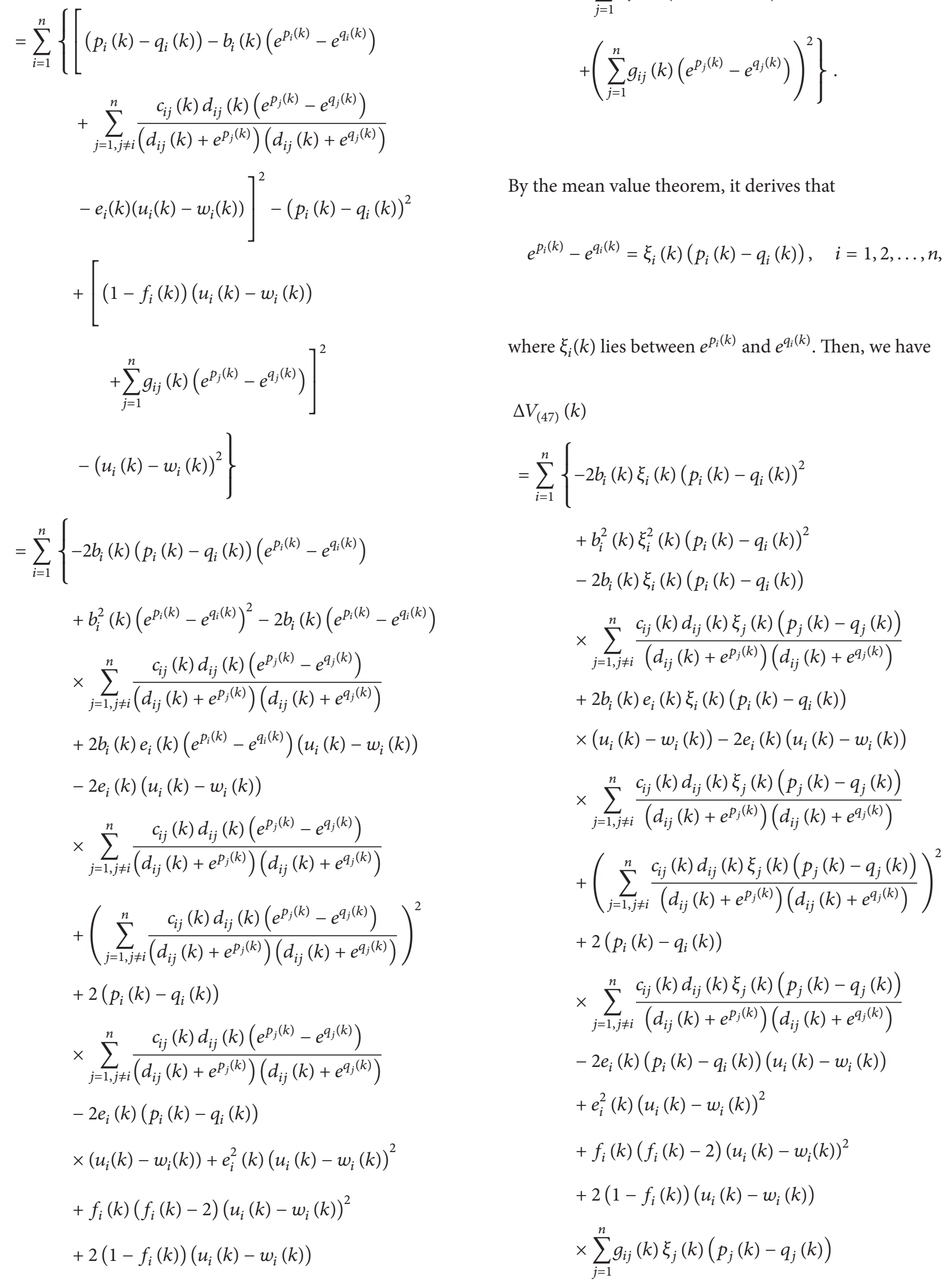

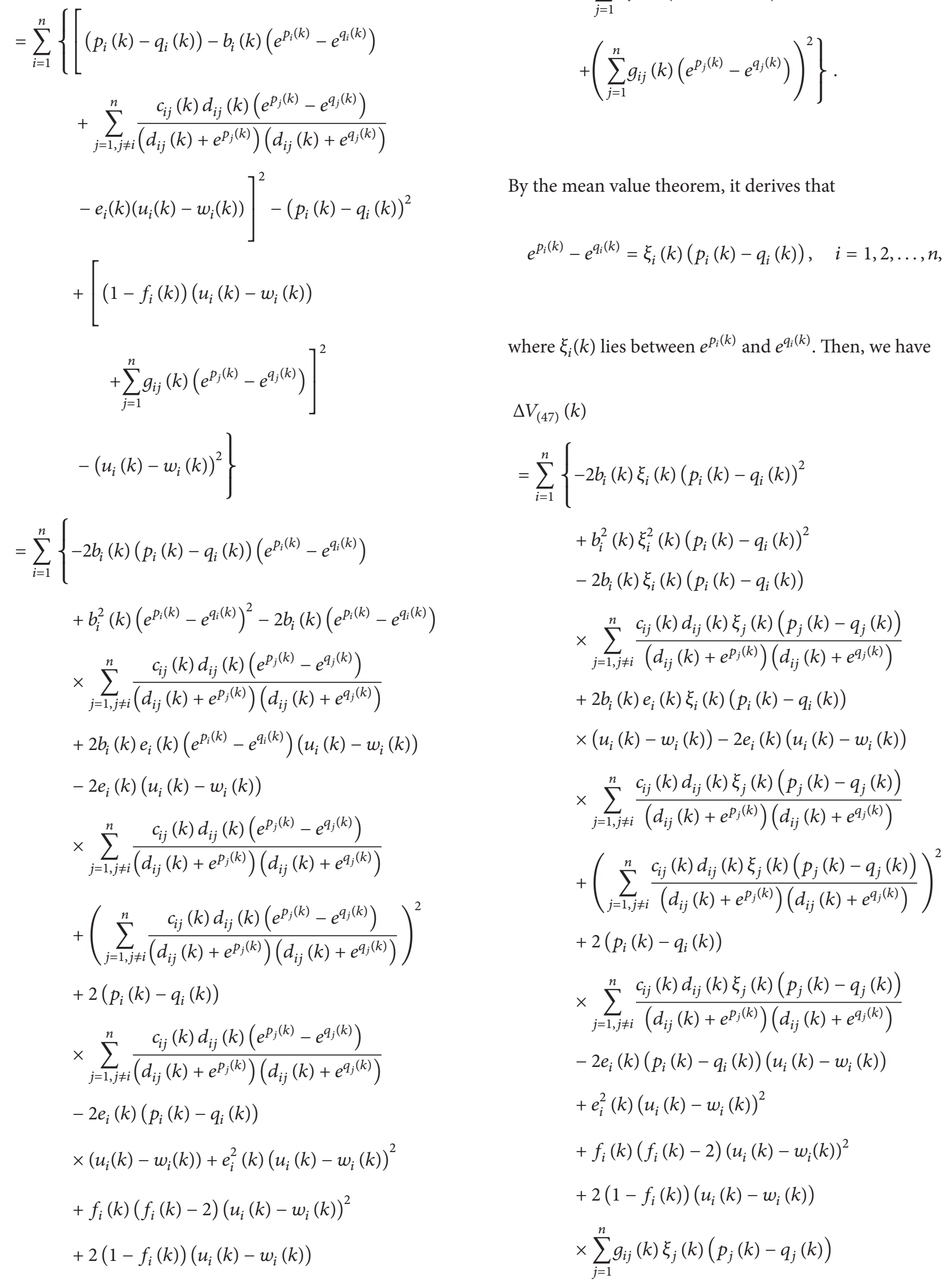

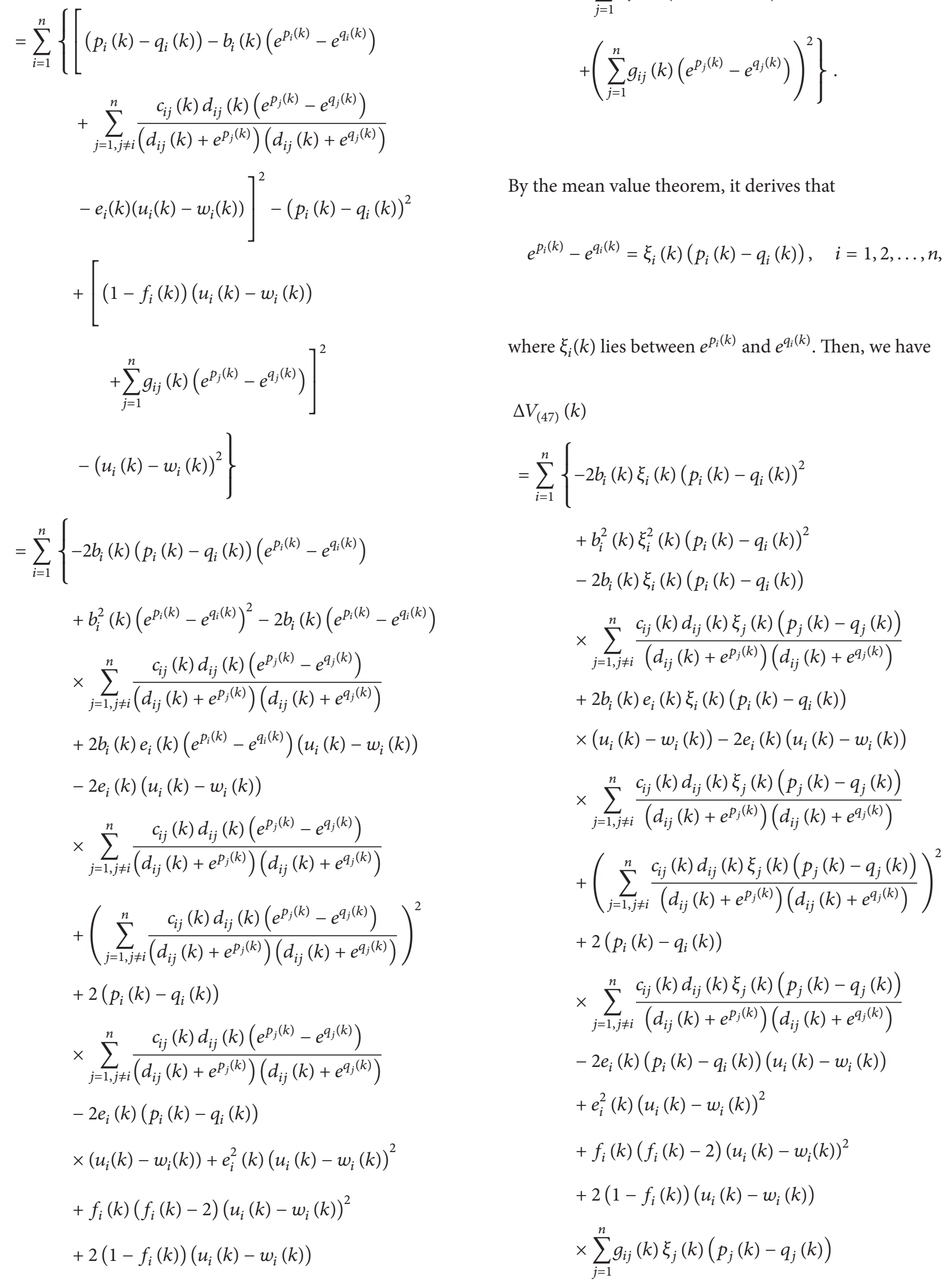

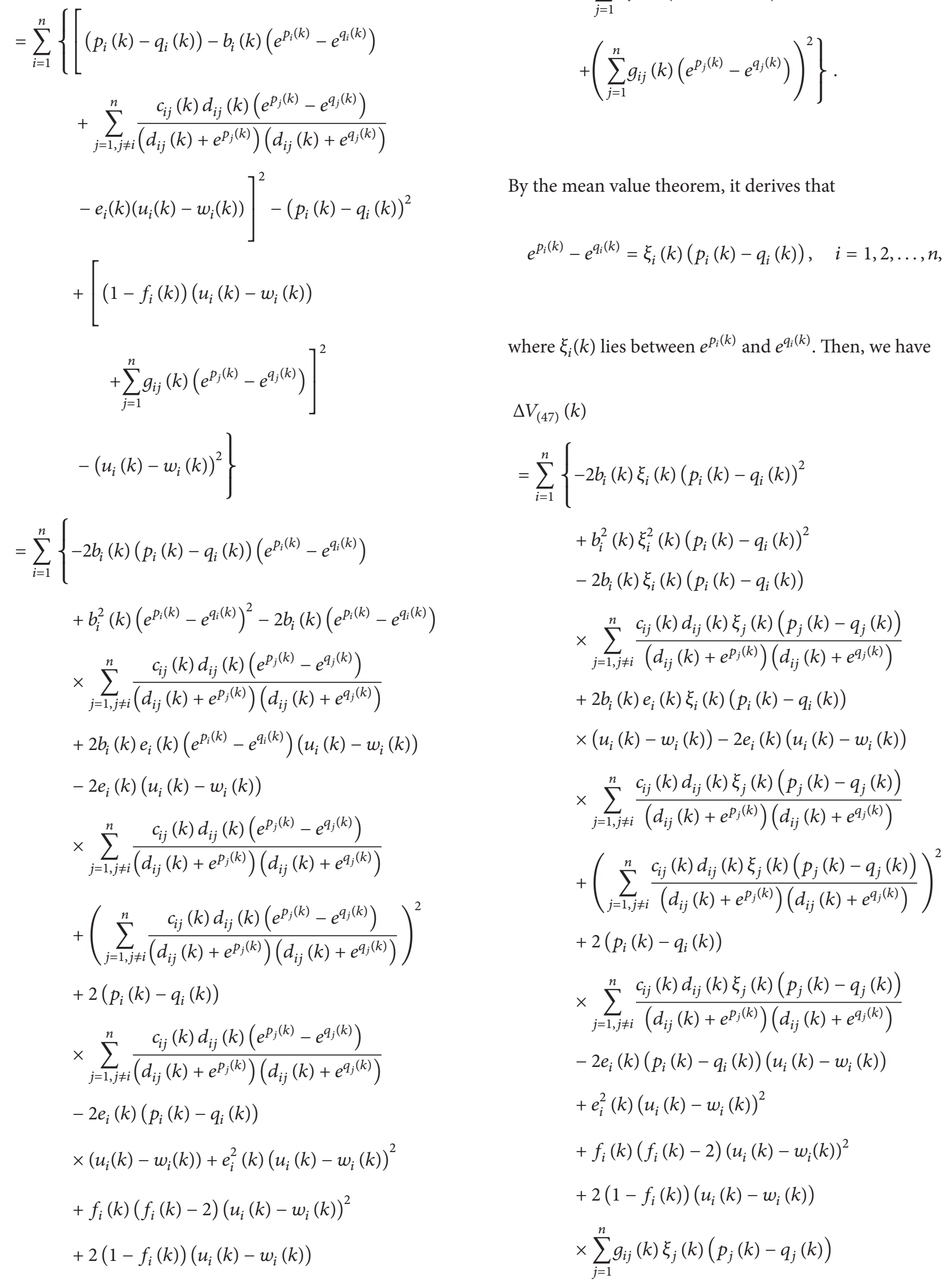

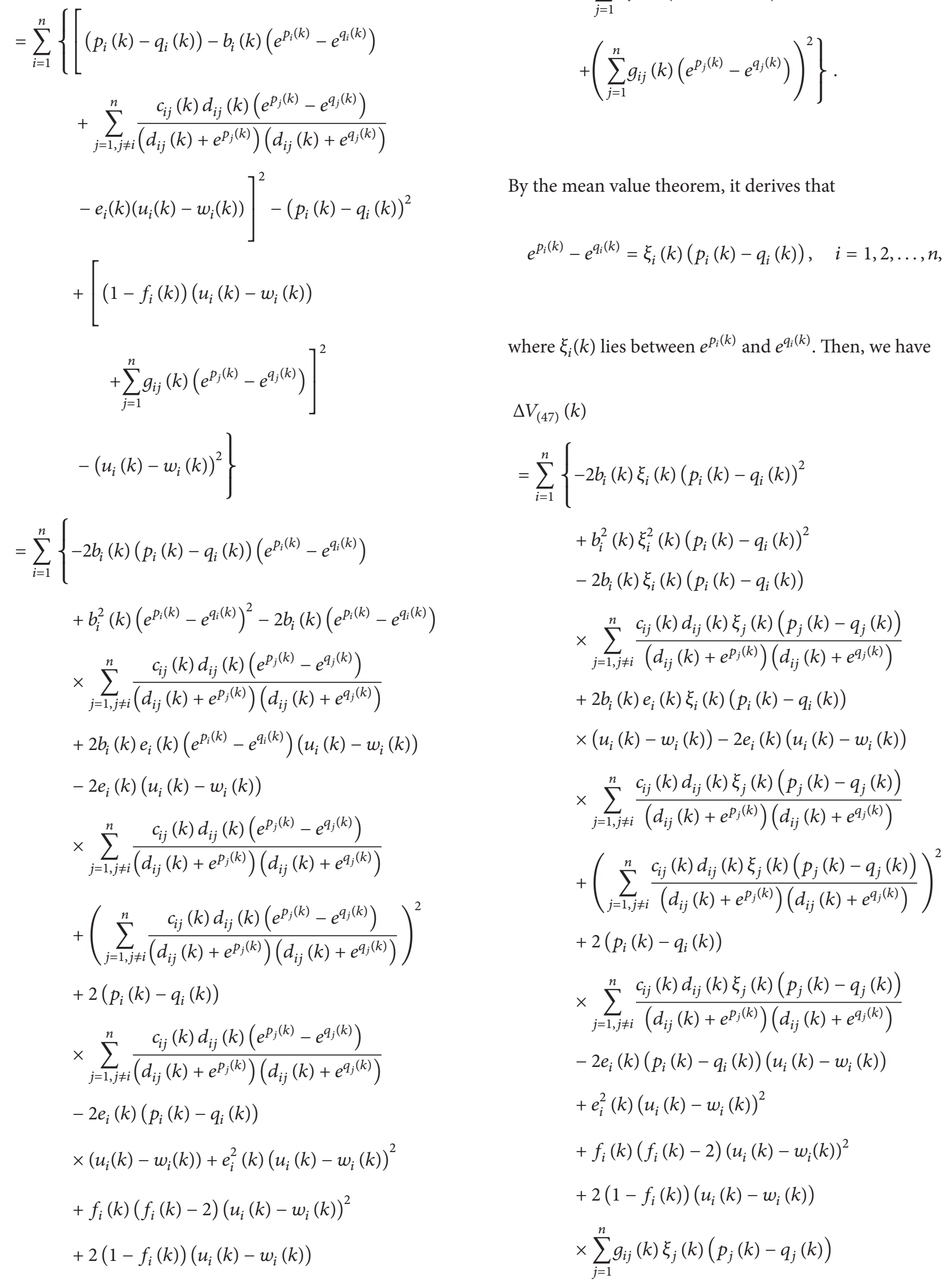

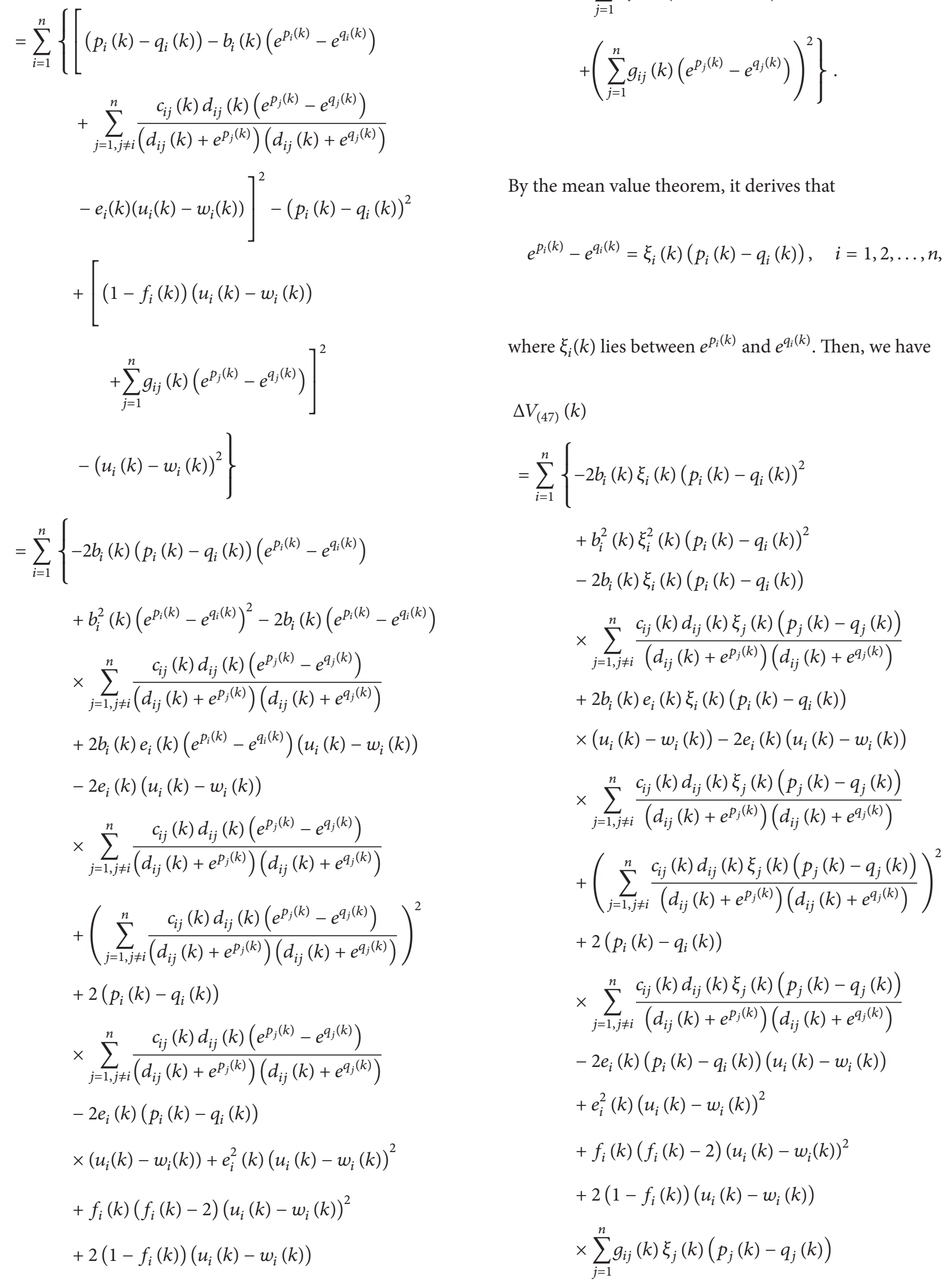

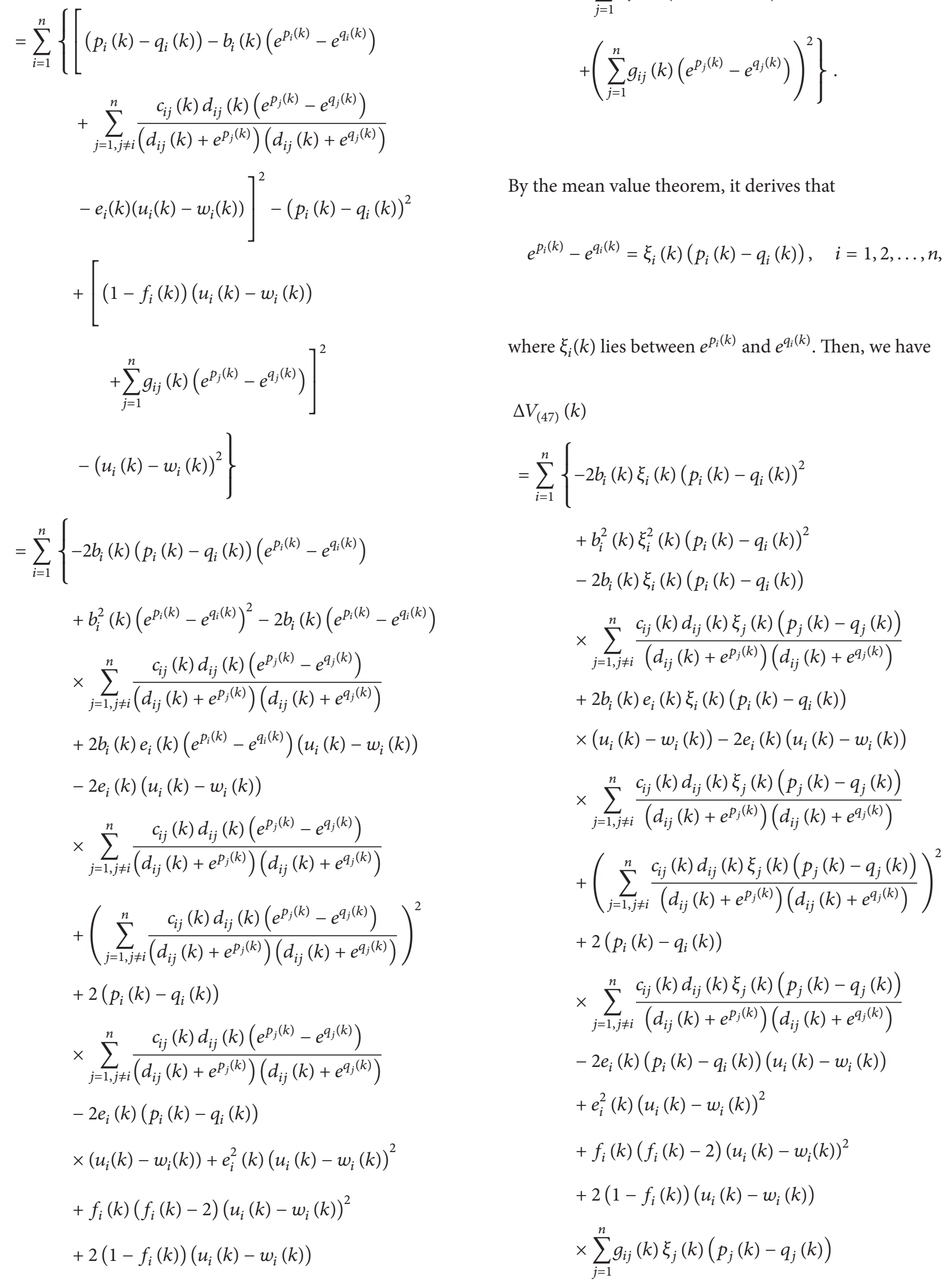

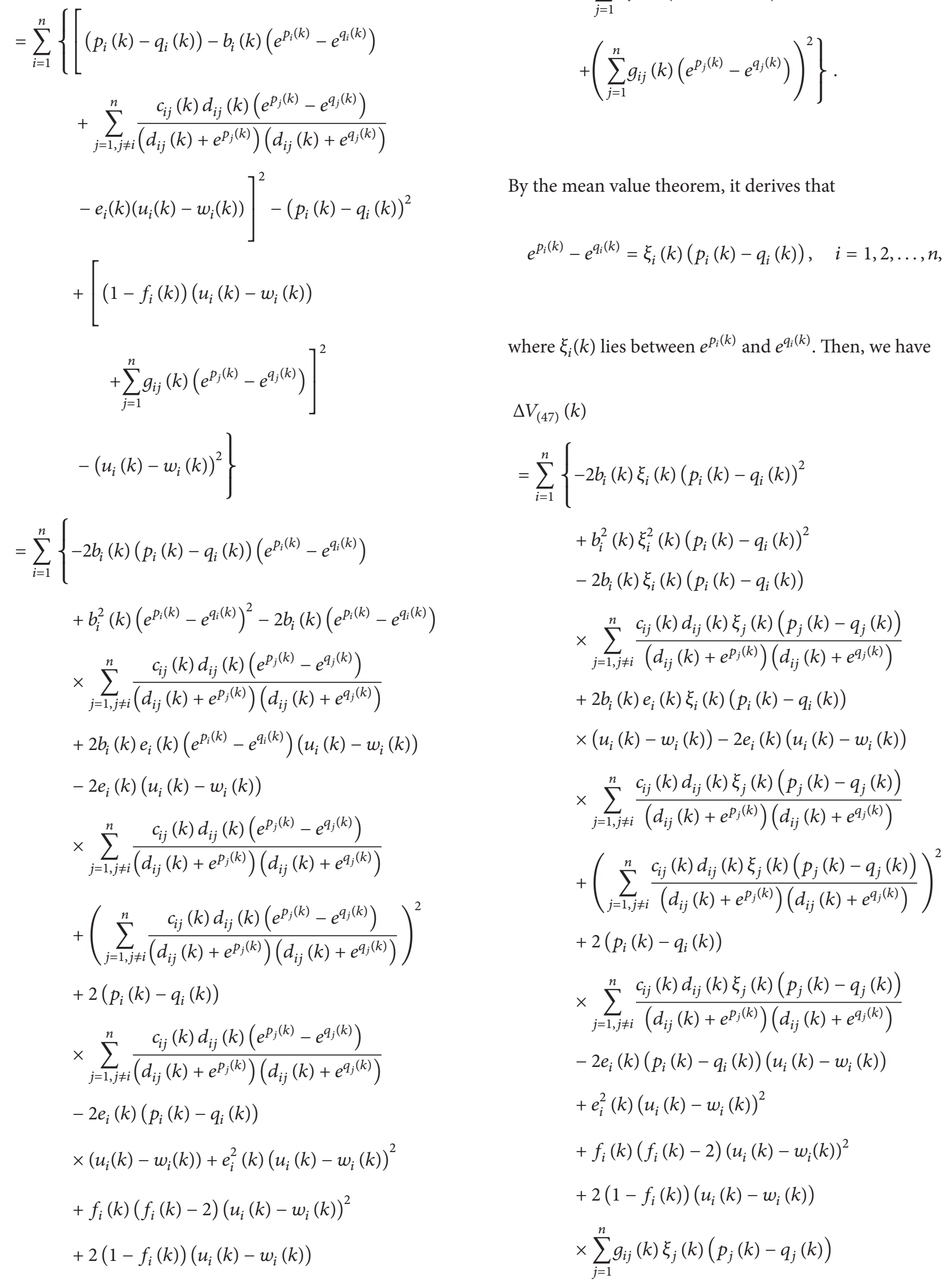

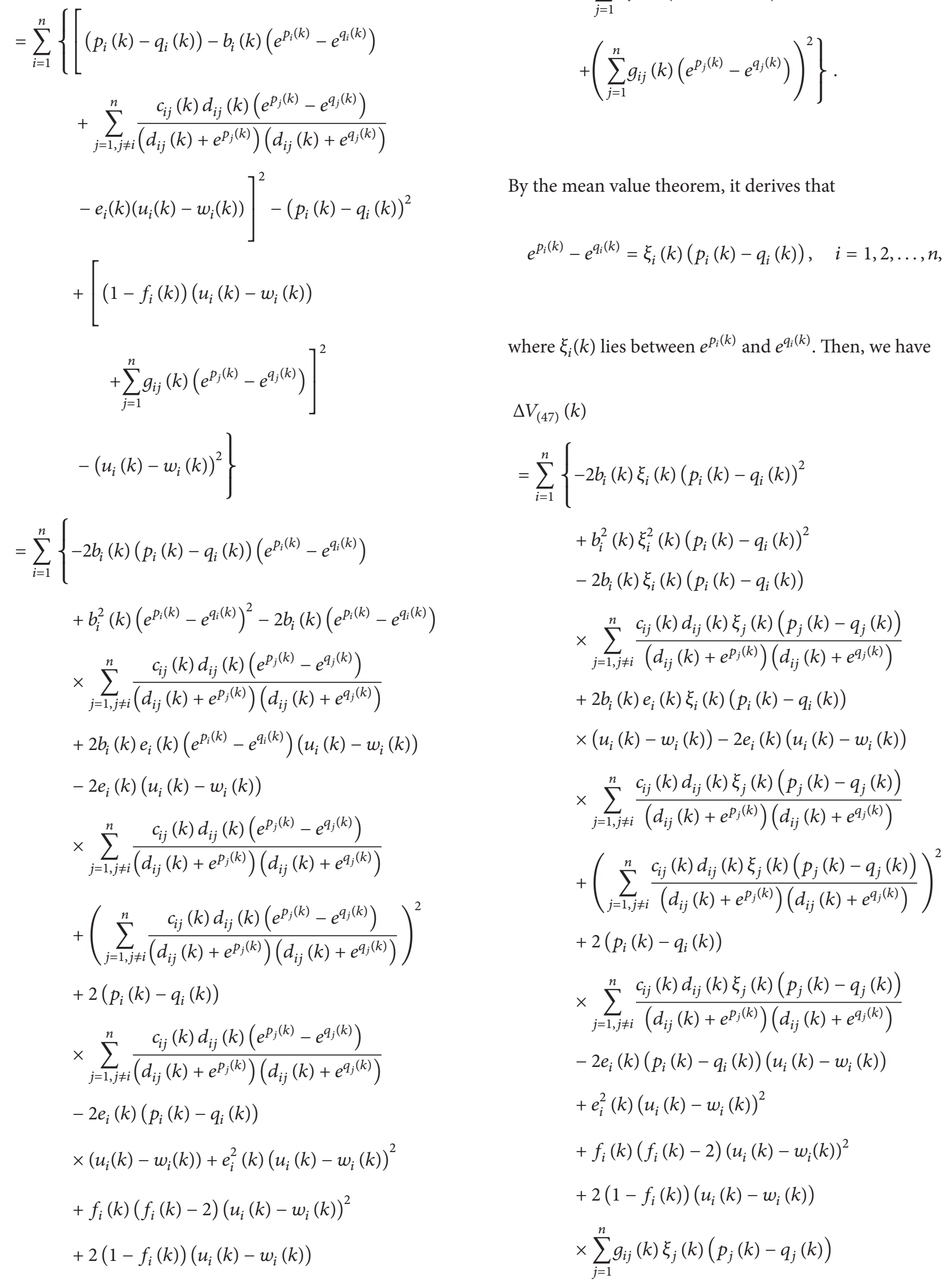

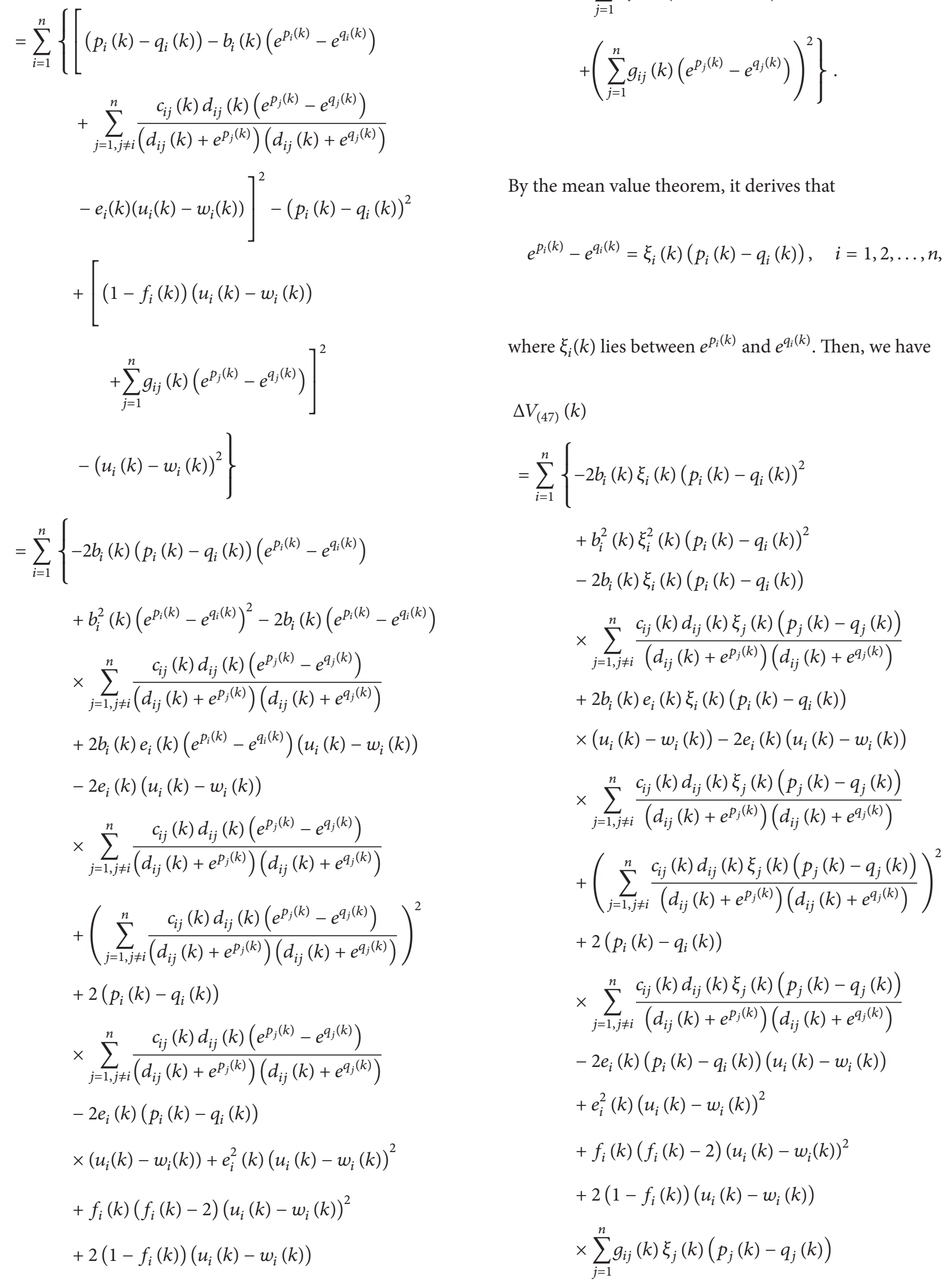

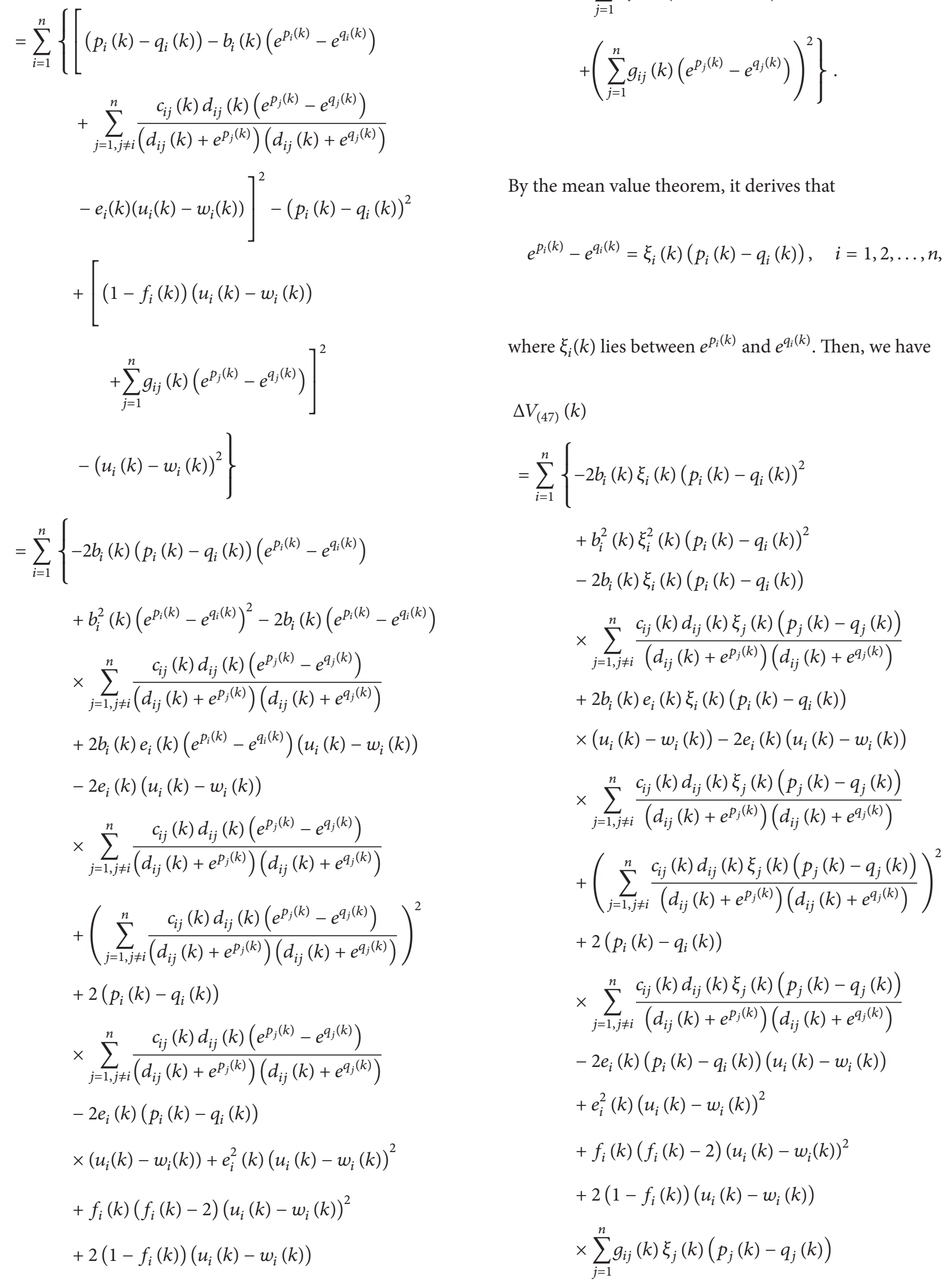

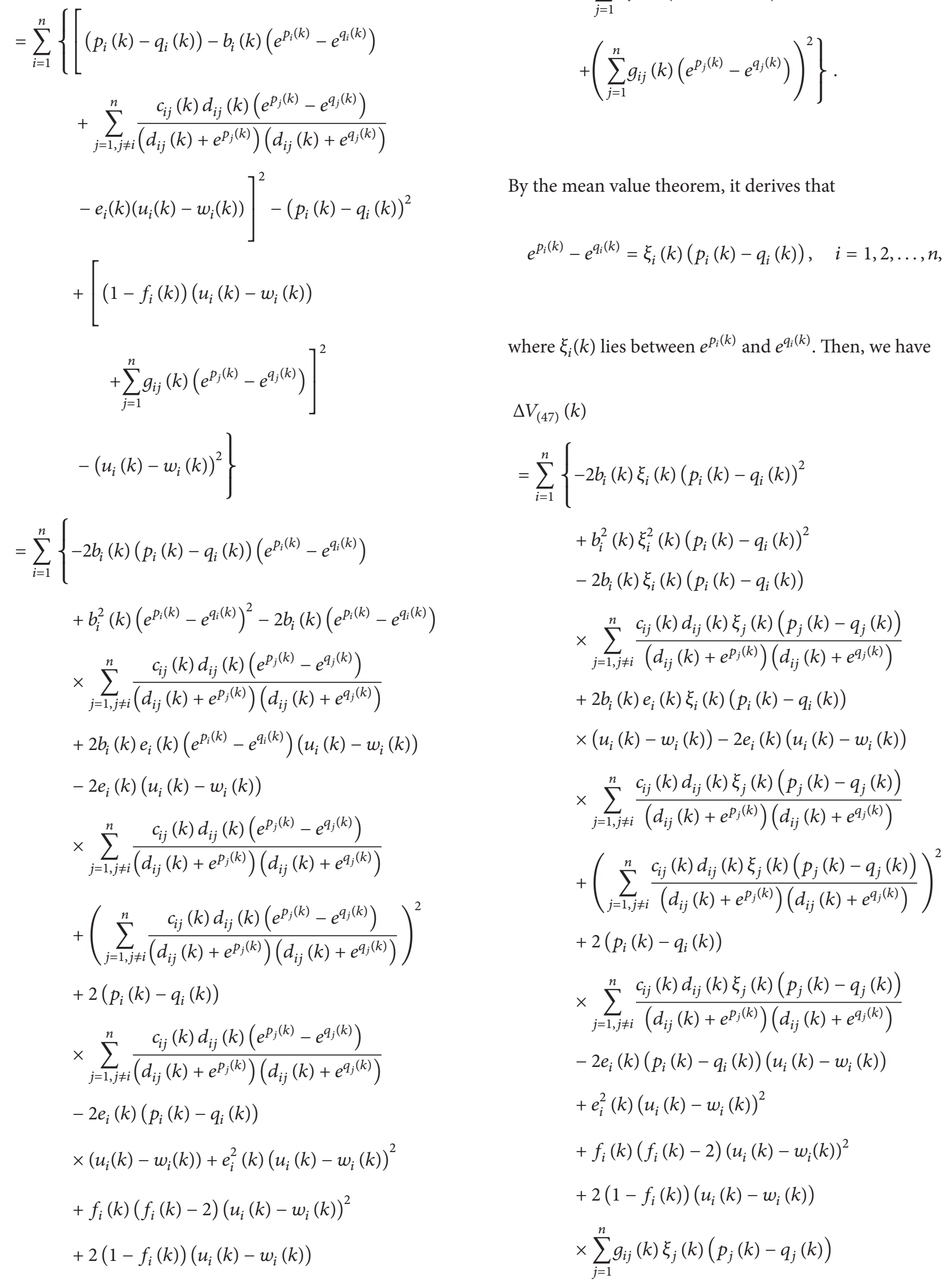

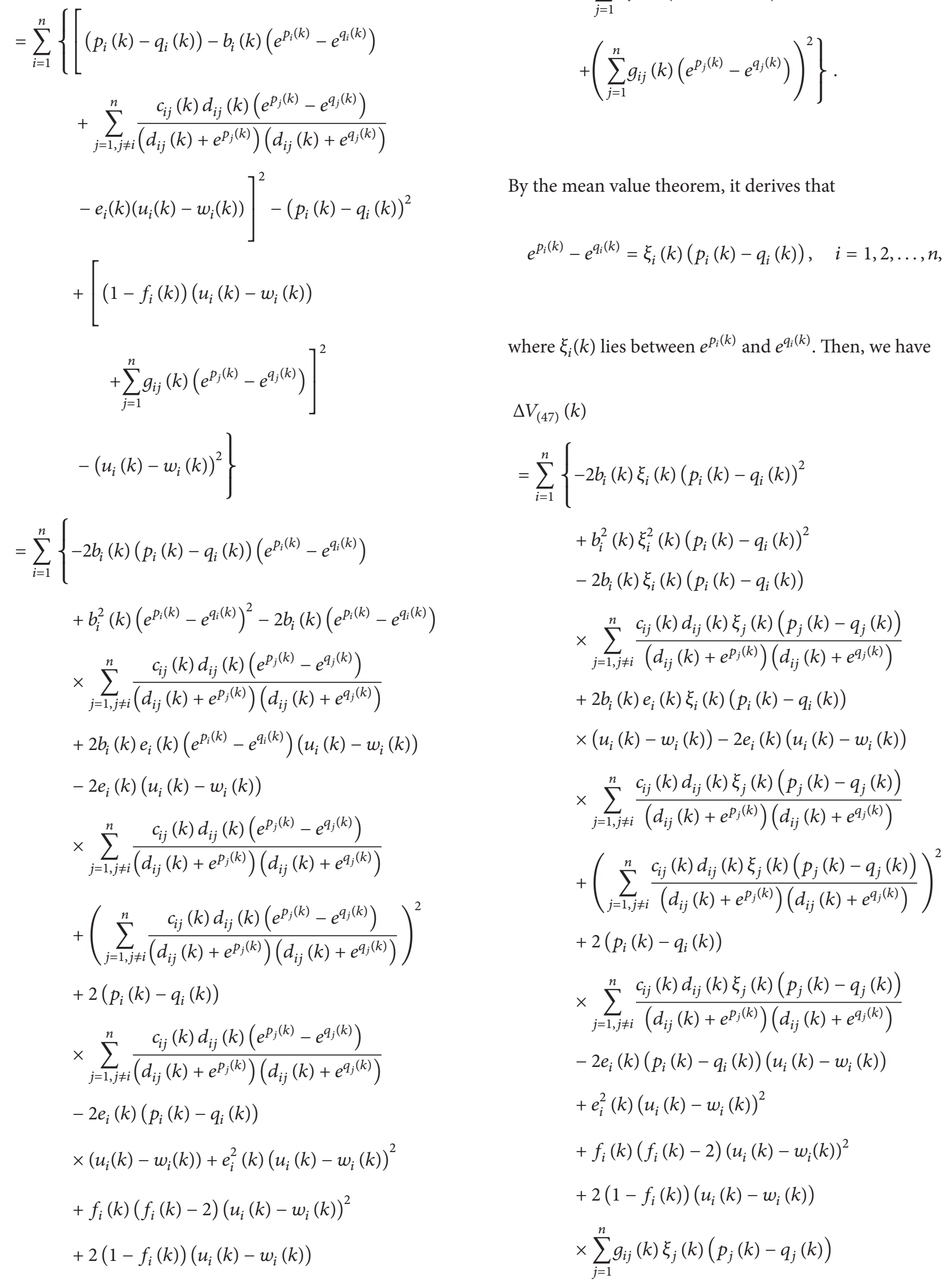

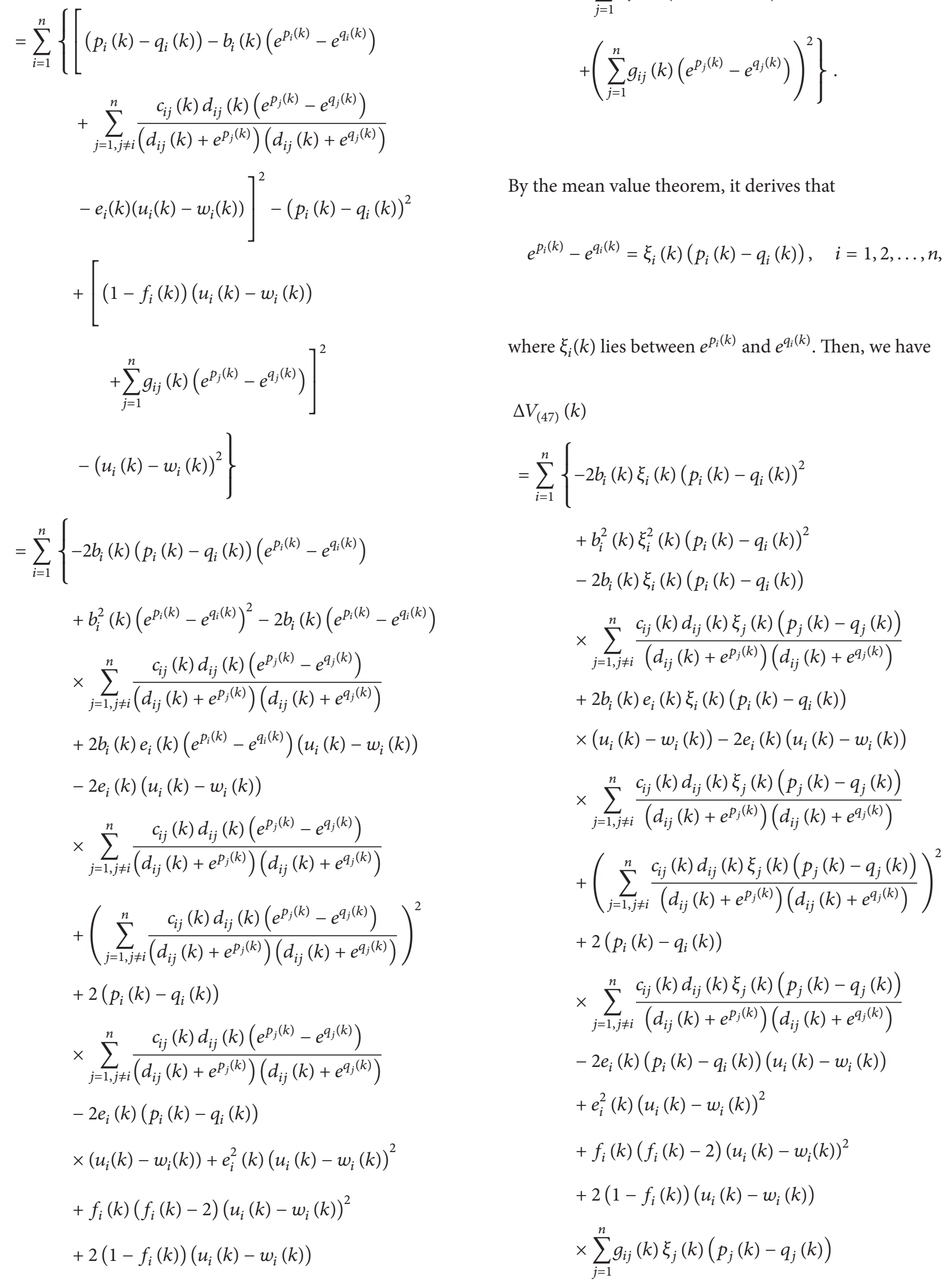

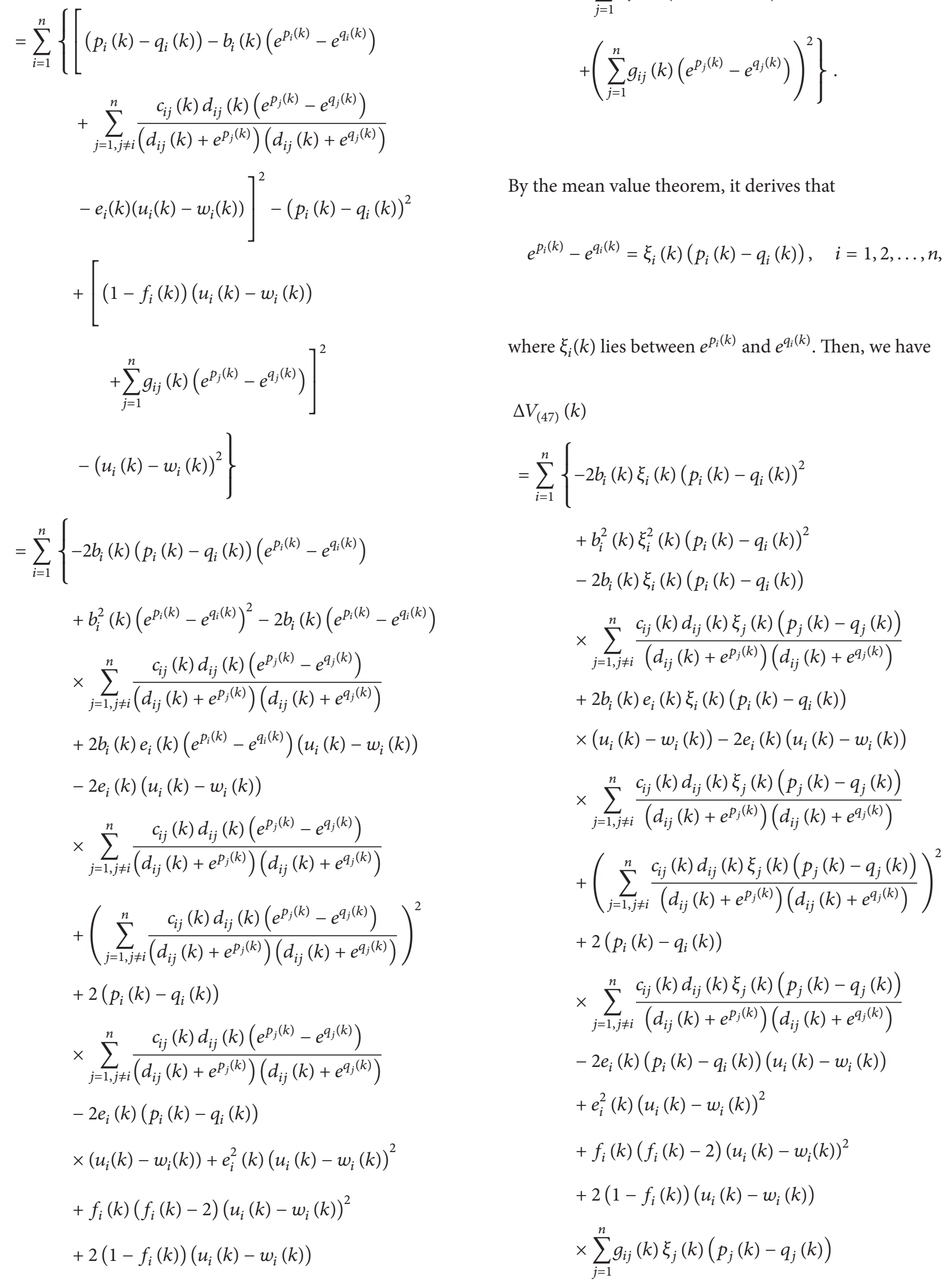

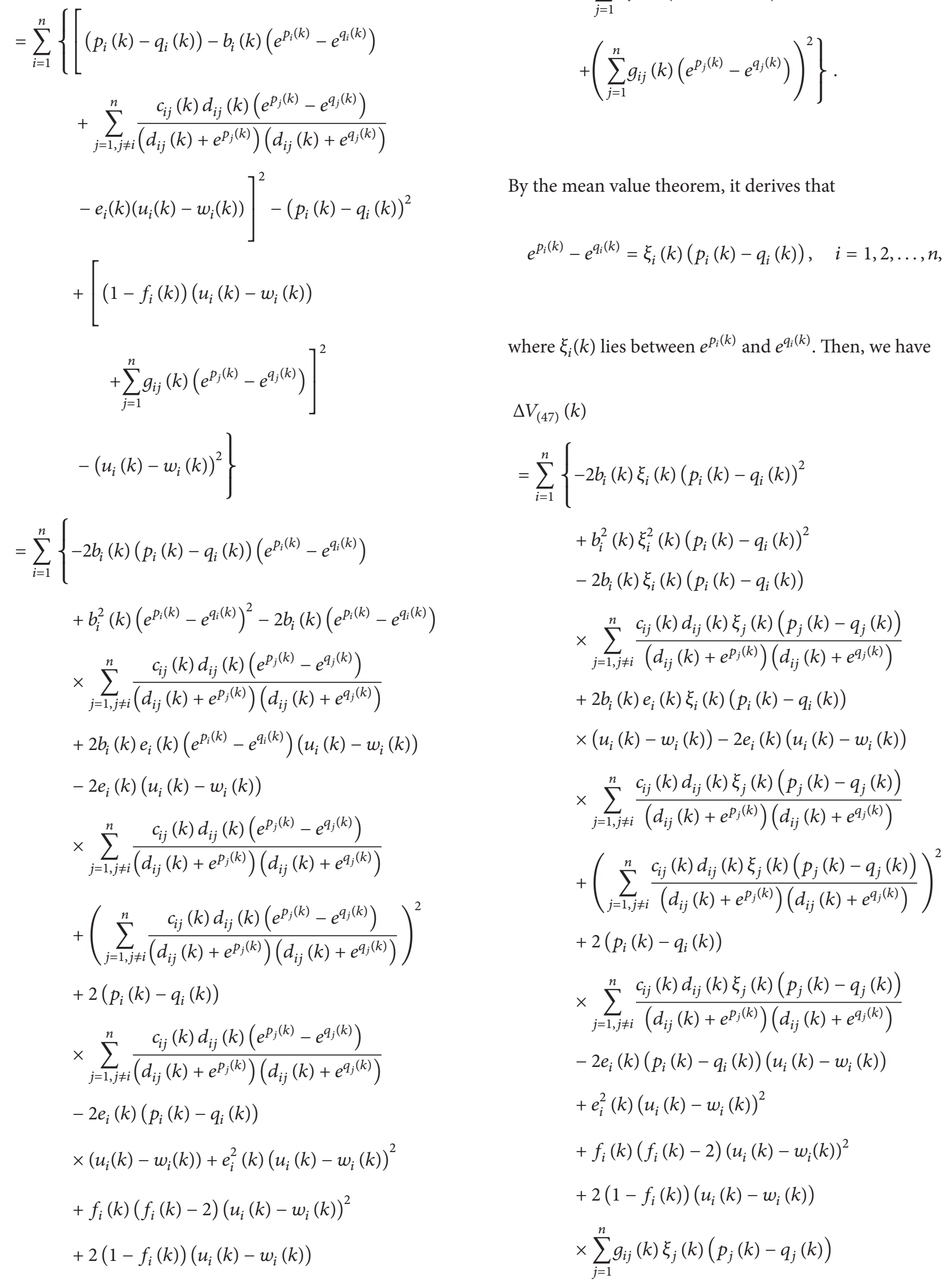

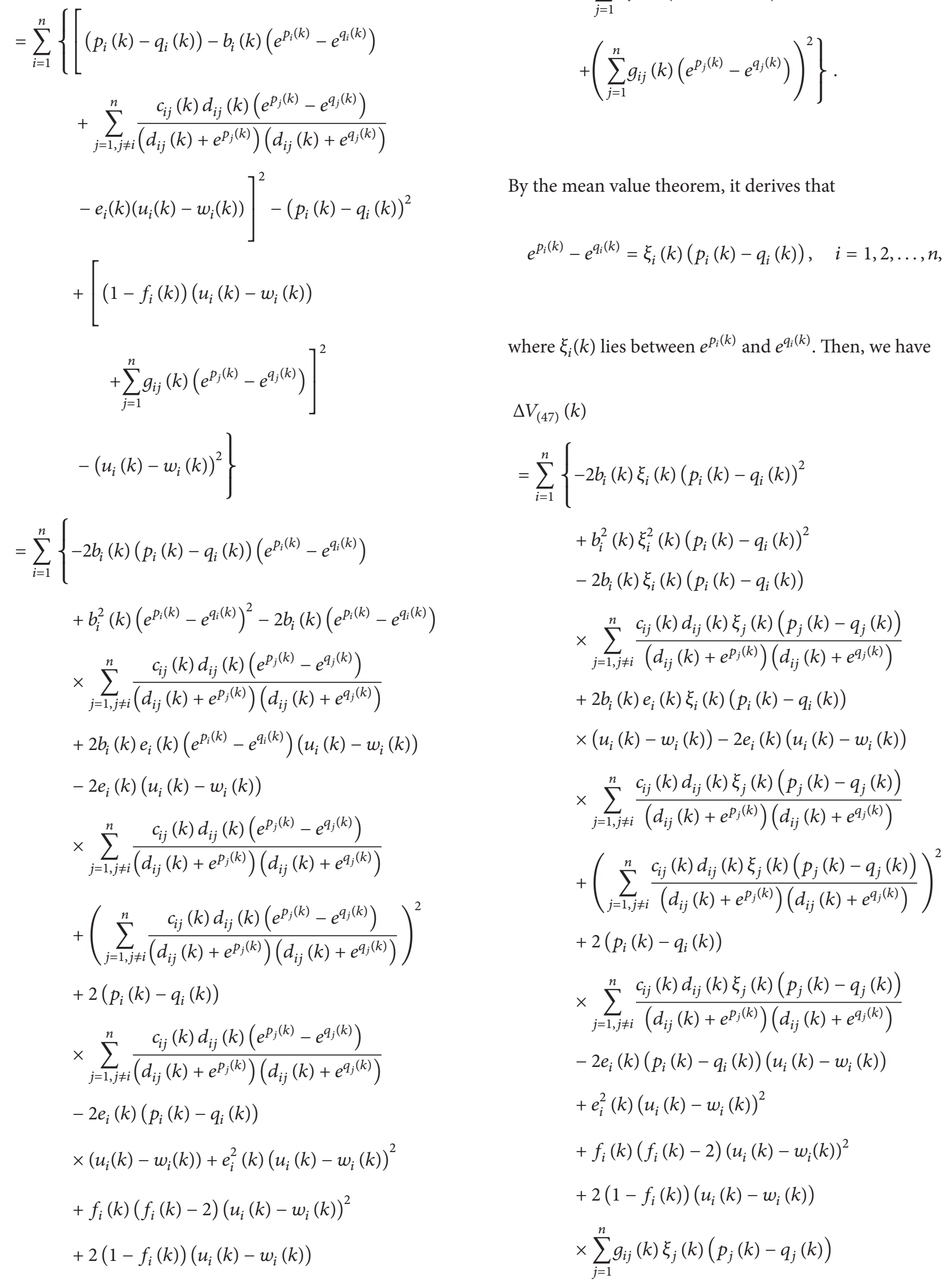

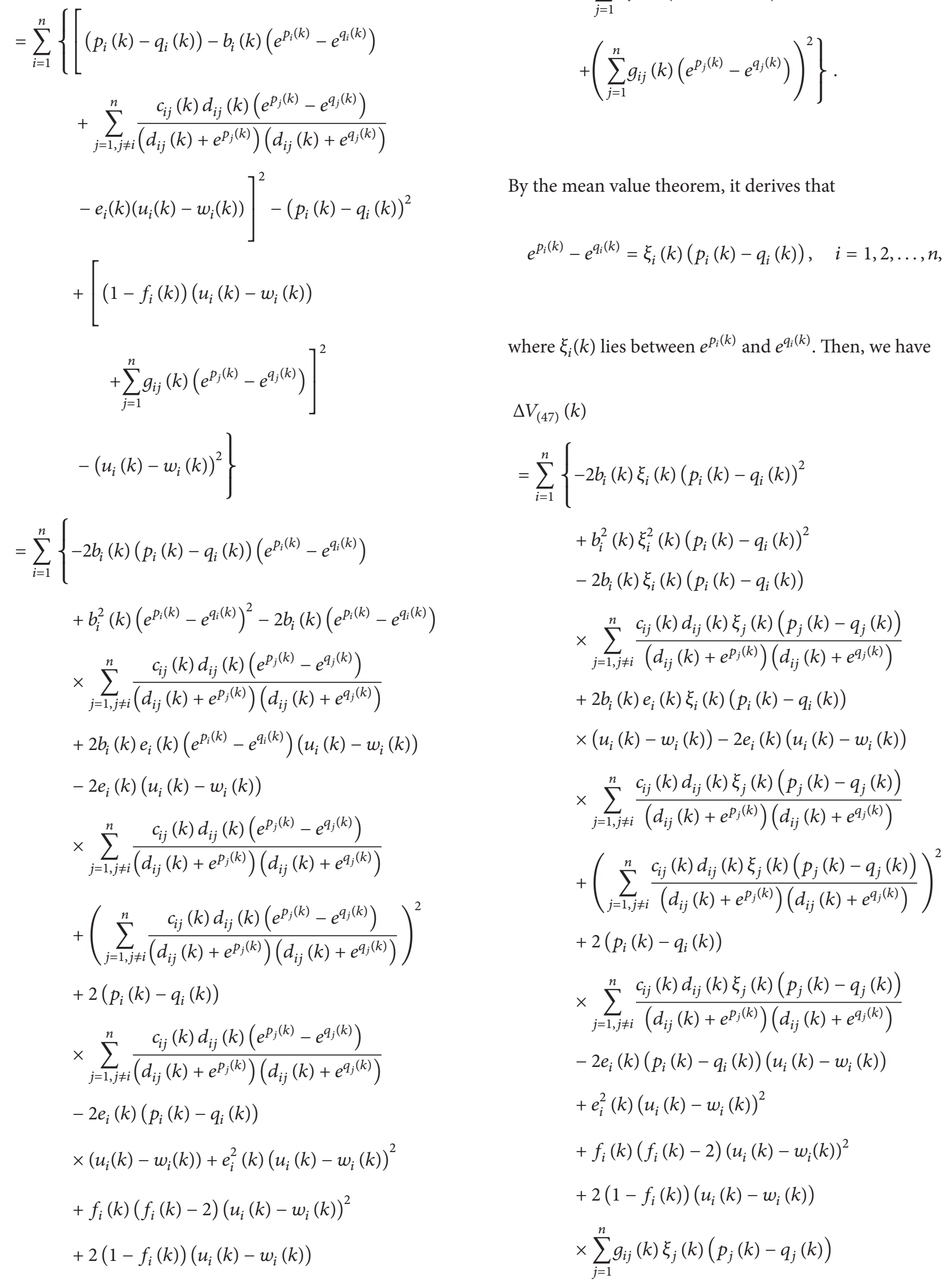

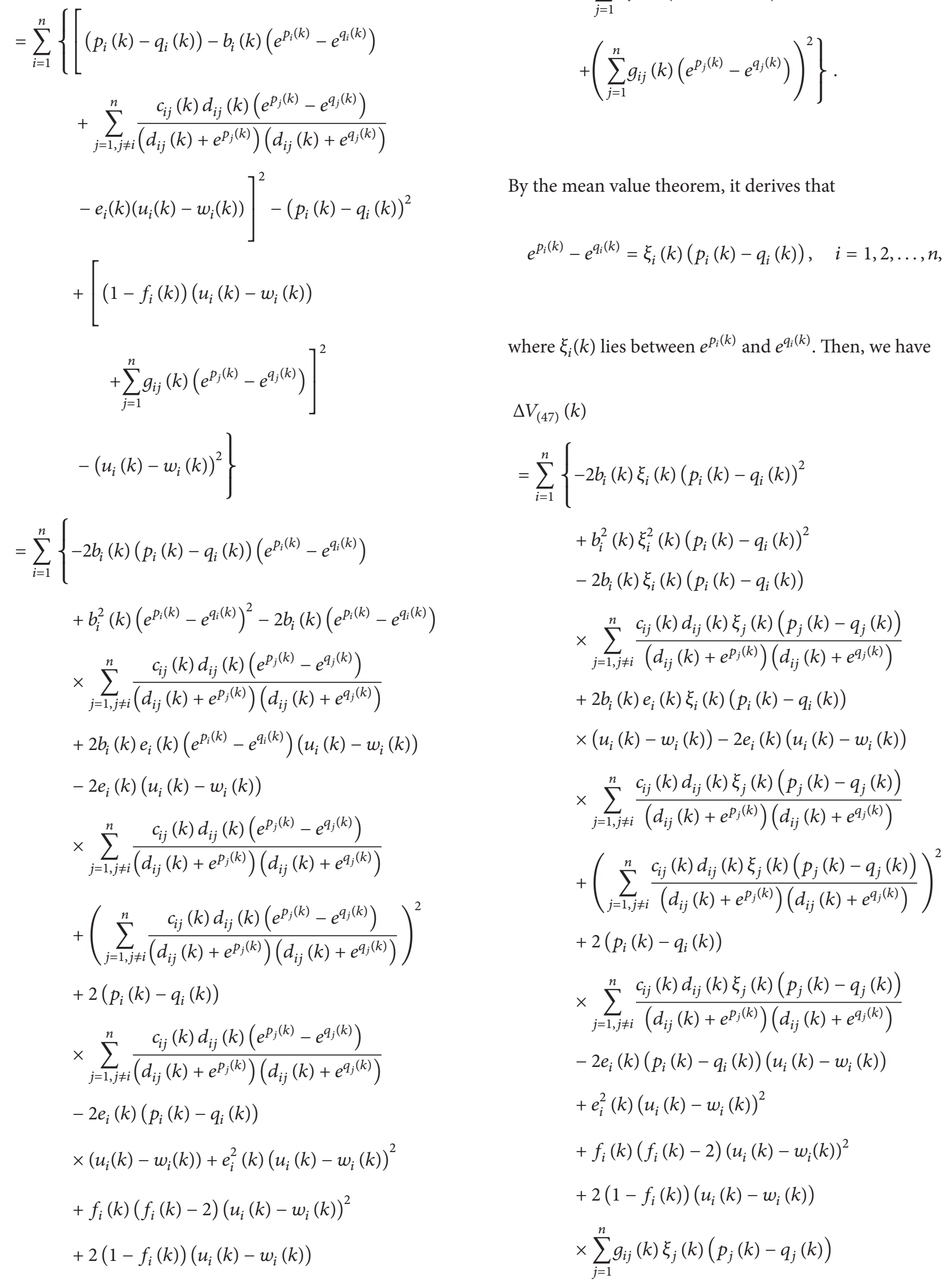

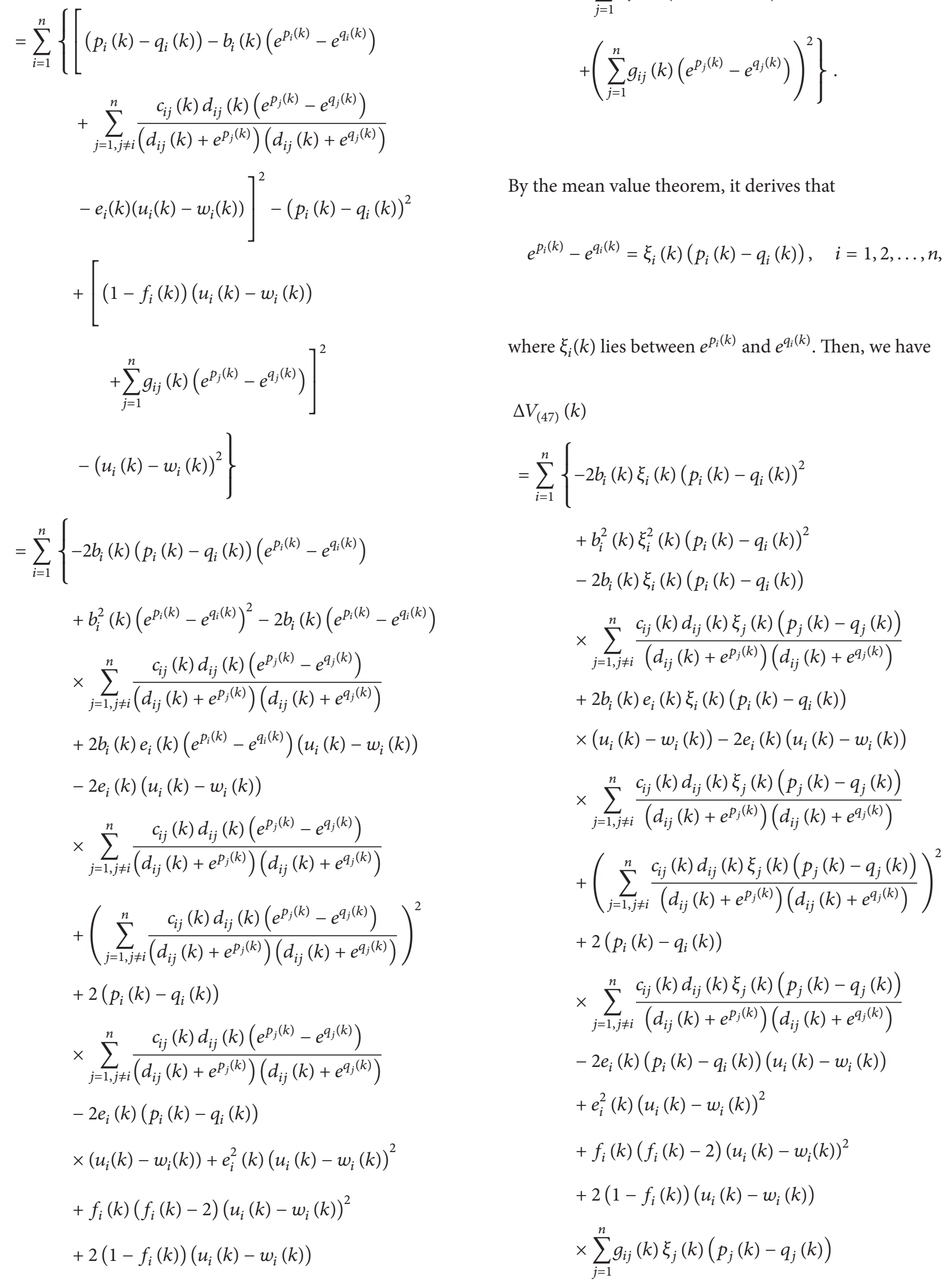

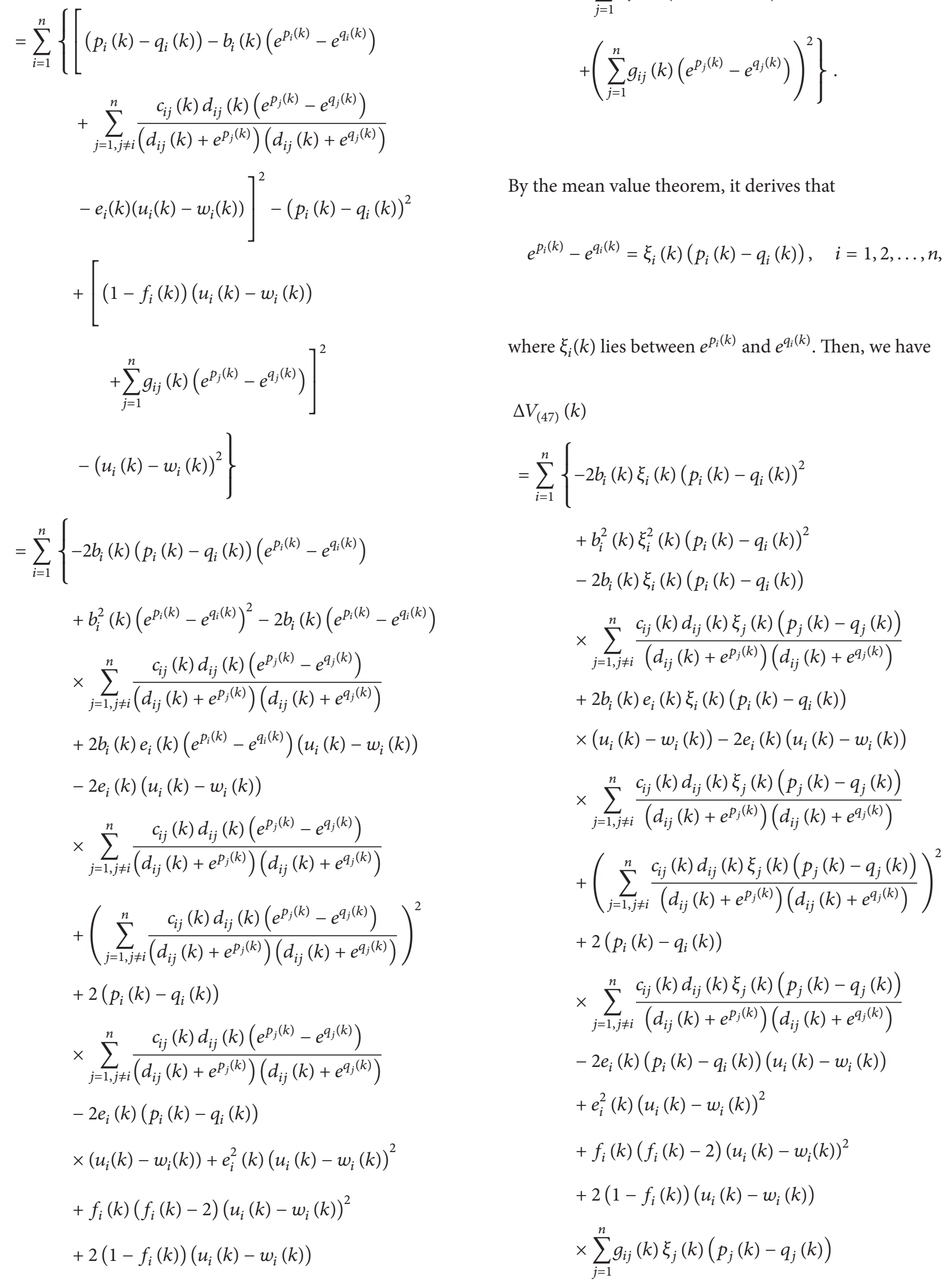

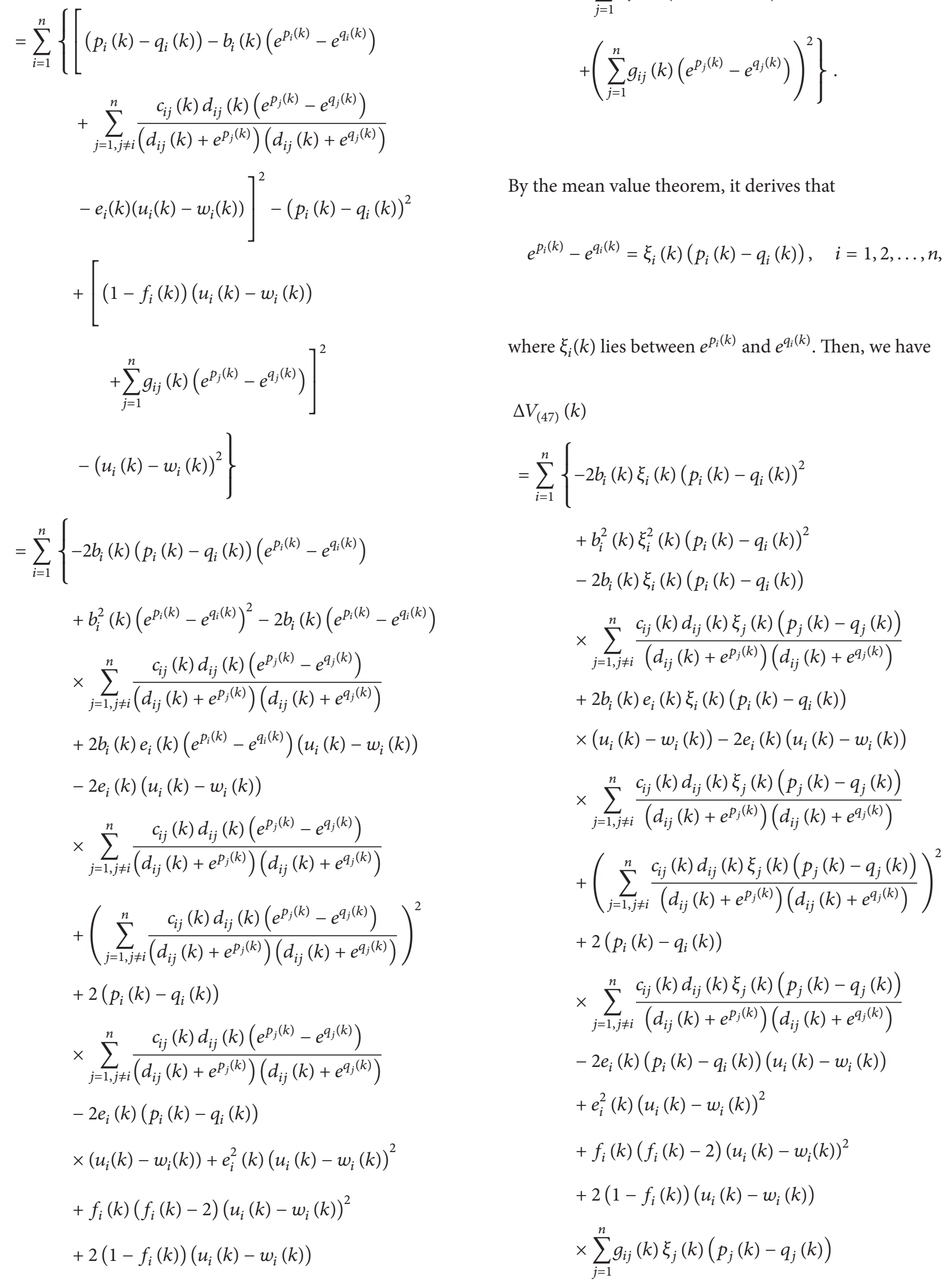

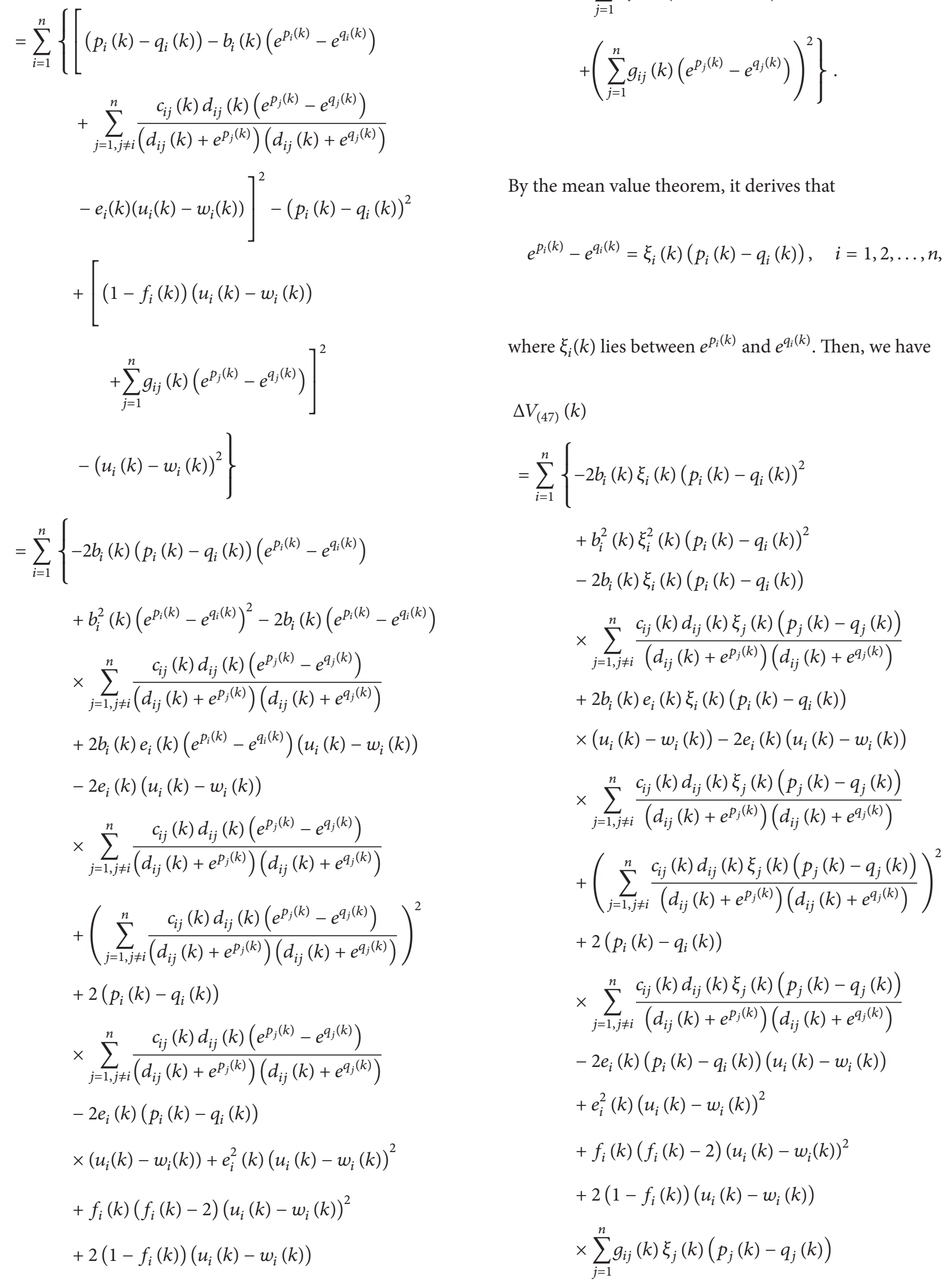




$$
\begin{aligned}
& \left.+\left(\sum_{j=1}^{n} g_{i j}(k) \xi_{j}(k)\left(p_{j}(k)-q_{j}(k)\right)\right)^{2}\right\} \\
& =\sum_{i=1}^{n}\left\{\left(-2 b_{i}(k) \xi_{i}(k)+b_{i}^{2}(k) \xi_{i}^{2}(k)+\sum_{j=1}^{n} g_{j i}^{2}(k) \xi_{i}^{2}(k)\right.\right. \\
& \left.+\sum_{j=1, j \neq i}^{n} \frac{c_{j i}^{2}(k) d_{j i}^{2}(k) \xi_{i}^{2}(k)}{\left(d_{j i}(k)+e^{p_{i}(k)}\right)^{2}\left(d_{j i}(k)+e^{q_{i}(k)}\right)^{2}}\right) \\
& \times\left(p_{i}(k)-q_{i}(k)\right)^{2} \\
& +2 \sum_{j=1, j \neq i}^{n}\left(\frac{\left(1-2 b_{i}(k) \xi_{i}(k)\right) c_{i j}(k) d_{i j}(k) \xi_{j}(k)}{\left(d_{i j}(k)+e^{p_{j}(k)}\right)\left(d_{i j}(k)+e^{q_{j}(k)}\right)}\right. \\
& +\frac{1}{2} \\
& \times \sum_{l=1, l \neq i, j}^{n} \frac{c_{l i}(k) d_{l i}(k) \xi_{i}(k)}{\left(d_{l i}(k)+e^{p_{i}(k)}\right)\left(d_{l i}(k)+e^{q_{i}(k)}\right)} \\
& \times \frac{c_{l j}(k) d_{l j}(k) \xi_{j}(k)}{\left(d_{l j}(k)+e^{p_{j}(k)}\right)\left(d_{l j}(k)+e^{q_{j}(k)}\right)} \\
& \left.+\frac{1}{2} \sum_{l=1}^{n} g_{l i}(k) g_{l j}(k) \xi_{i}(k) \xi_{j}(k)\right) \\
& \times\left(p_{i}(k)-q_{i}(k)\right)\left(p_{j}(k)-q_{j}(k)\right) \\
& +2\left[e_{i}(k)\left(b_{i}(k) \xi_{i}(k)-1\right)\right. \\
& \left.+g_{i i}(k) \xi_{i}(k)\left(1-f_{i}(k)\right)\right] \\
& \times\left(p_{i}(k)-q_{i}(k)\right)\left(u_{i}(k)-w_{i}(k)\right) \\
& +2 \sum_{j=1, j \neq i}^{n}\left[\left(1-f_{i}(k)\right) g_{i j}(k) \xi_{j}(k)-e_{i}(k)\right. \\
& \left.\times \frac{c_{i j}(k) d_{i j}(k) \xi_{j}(k)}{\left(d_{i j}(k)+e^{p_{j}(k)}\right)\left(d_{i j}(k)+e^{q_{j}(k)}\right)}\right] \\
& \times\left(p_{j}(k)-q_{j}(k)\right)\left(u_{i}(k)-w_{i}(k)\right) \\
& \left.+\left[e_{i}^{2}(k)+f_{i}(k)\left(f_{i}(k)-2\right)\right]\left(u_{i}(k)-w_{i}(k)\right)^{2}\right\} \\
& \leq \sum_{i=1}^{n}\left\{\left(-2 b_{i}(k) \xi_{i}(k)+b_{i}^{2}(k) \xi_{i}^{2}(k)\right.\right. \\
& +\sum_{j=1}^{n} g_{j i}^{2}(k) \xi_{i}^{2}(k)
\end{aligned}
$$$$
\left.+\sum_{j=1, j \neq i}^{n} \frac{c_{j i}^{2}(k) d_{j i}^{2}(k) \xi_{i}^{2}(k)}{\left(d_{j i}(k)+e^{p_{i}(k)}\right)^{2}\left(d_{j i}(k)+e^{q_{i}(k)}\right)^{2}}\right)
$$$$
\times\left(p_{i}(k)-q_{i}(k)\right)^{2}+2
$$$$
\times \mid \sum_{j=1, j \neq i}^{n}\left(\frac{\left(1-2 b_{i}(k) \xi_{i}(k)\right) c_{i j}(k) d_{i j}(k) \xi_{j}(k)}{\left(d_{i j}(k)+e^{p_{j}(k)}\right)\left(d_{i j}(k)+e^{q_{j}(k)}\right)}+\frac{1}{2}\right.
$$$$
\times \sum_{l=1, l \neq i, j}^{n} \frac{c_{l i}(k) d_{l i}(k) \xi_{i}(k)}{\left(d_{l i}(k)+e^{p_{i}(k)}\right)\left(d_{l i}(k)+e^{q_{i}(k)}\right)}
$$$$
\times \frac{c_{l j}(k) d_{l j}(k) \xi_{j}(k)}{\left(d_{l j}(k)+e^{p_{j}(k)}\right)\left(d_{l j}(k)+e^{q_{j}(k)}\right)}
$$$$
\left.+\frac{1}{2} \sum_{l=1}^{n} g_{l i}(k) g_{l j}(k) \xi_{i}(k) \xi_{j}(k)\right)
$$$$
\times\left(p_{i}(k)-q_{i}(k)\right)\left(p_{j}(k)-q_{j}(k)\right)
$$$$
+2 \mid\left[e_{i}(k)\left(b_{i}(k) \xi_{i}(k)-1\right)\right.
$$$$
\left.+g_{i i}(k) \xi_{i}(k)\left(1-f_{i}(k)\right)\right]
$$$$
\times\left(p_{i}(k)-q_{i}(k)\right)\left(u_{i}(k)-w_{i}(k)\right)
$$$$
+2 \mid \sum_{j=1, j \neq i}^{n}\left[\left(1-f_{i}(k)\right) g_{i j}(k) \xi_{j}(k)-e_{i}(k)\right.
$$$$
\left.\times \frac{c_{i j}(k) d_{i j}(k) \xi_{j}(k)}{\left(d_{i j}(k)+e^{p_{j}(k)}\right)\left(d_{i j}(k)+e^{q_{j}(k)}\right)}\right]
$$$$
\times\left(p_{j}(k)-q_{j}(k)\right)\left(u_{i}(k)-w_{i}(k)\right)
$$$$
+\left[e_{i}^{2}(k)+f_{i}(k)\left(f_{i}(k)-2\right)\right]
$$$$
\left.\times\left(u_{i}(k)-w_{i}(k)\right)^{2}\right\} \text {. }
$$

Then, we have

$$
\Delta V_{(47)}(k) \leq \sum_{i=1}^{n}\left\{V_{i 1}+V_{i 2}+V_{i 3}+V_{i 4}+V_{i 5}\right\},
$$

where

$$
\begin{aligned}
V_{i 1}=( & -2 b_{i}(k) \xi_{i}(k)+b_{i}^{2}(k) \xi_{i}^{2}(k)+\sum_{j=1}^{n} g_{j i}^{2}(k) \xi_{i}^{2}(k) \\
& \left.+\sum_{j=1, j \neq i}^{n} \frac{c_{j i}^{2}(k) d_{j i}^{2}(k) \xi_{i}^{2}(k)}{\left(d_{j i}(k)+e^{p_{i}(k)}\right)^{2}\left(d_{j i}(k)+e^{q_{i}(k)}\right)^{2}}\right)
\end{aligned}
$$


Discrete Dynamics in Nature and Society

9

$$
\begin{aligned}
& \times\left(p_{i}(k)-q_{i}(k)\right)^{2} \\
& V_{i 5}=\left[e_{i}^{2}(k)+f_{i}(k)\left(f_{i}(k)-2\right)\right]\left(u_{i}(k)-w_{i}(k)\right)^{2} \\
& \leq\left(-2 b_{i}^{l} m_{i}+b_{i}^{u 2} M_{i}^{2}+\sum_{j=1}^{n} g_{j i}^{u 2} M_{i}^{2}+\sum_{j=1, j \neq i}^{n} c_{j i}^{u 2}\right) \\
& \leq\left[e_{i}^{u 2}+f_{i}^{l}\left(f_{i}^{u}-2\right)\right]\left(u_{i}(k)-w_{i}(k)\right)^{2} . \\
& \times\left(p_{i}(k)-q_{i}(k)\right)^{2}, \\
& V_{i 2}=2 \mid \sum_{j=1, j \neq i}^{n}\left(\frac{\left(1-2 b_{i}(k) \xi_{i}(k)\right) c_{i j}(k) d_{i j}(k) \xi_{j}(k)}{\left(d_{i j}(k)+e^{p_{j}(k)}\right)\left(d_{i j}(k)+e^{q_{j}(k)}\right)}\right. \\
& +\frac{1}{2} \sum_{l=1, l \neq i, j}^{n} \frac{c_{l i}(k) d_{l i}(k) \xi_{i}(k)}{\left(d_{l i}(k)+e^{p_{i}(k)}\right)\left(d_{l i}(k)+e^{q_{i}(k)}\right)} \\
& \times \frac{c_{l j}(k) d_{l j}(k) \xi_{j}(k)}{\left(d_{l j}(k)+e^{p_{j}(k)}\right)\left(d_{l j}(k)+e^{q_{j}(k)}\right)} \\
& \left.+\frac{1}{2} \sum_{l=1}^{n} g_{l i}(k) g_{l j}(k) \xi_{i}(k) \xi_{j}(k)\right) \\
& \times\left(p_{i}(k)-q_{i}(k)\right)\left(p_{j}(k)-q_{j}(k)\right) \\
& \leq \sum_{j=1, j \neq i}^{n}\left(\left(1+2 b_{i}^{u} M_{i}\right) c_{i j}^{u}+\frac{1}{2} \sum_{l=1, l \neq i, j}^{n} c_{l i}^{u} c_{l j}^{u}\right. \\
& \left.+\frac{1}{2} \sum_{l=1}^{n} g_{l i}^{u} g_{l j}^{u} M_{i} M_{j}\right) \\
& \times\left[\left(p_{i}(k)-q_{i}(k)\right)^{2}+\left(p_{j}(k)-q_{j}(k)\right)^{2}\right], \\
& \begin{array}{l}
\Delta V_{(47)}(k) \\
\leq \sum_{i=1}^{n}\left\{\left(-2 b_{i}^{l} m_{i}+b_{i}^{u 2} M_{i}^{2}+\sum_{j=1}^{n} g_{j i}^{u 2} M_{i}^{2}\right.\right. \\
+\sum_{j=1, j \neq i}^{n} c_{j i}^{u 2}+\left(1+2 b_{i}^{u} M_{i}\right) c_{i j}^{u}+\frac{1}{2} \sum_{l=1, l \neq i, j}^{n} c_{l i}^{u} c_{l j}^{u}
\end{array} \\
& \begin{array}{l}
\Delta V_{(47)}(k) \\
\leq \sum_{i=1}^{n}\left\{\left(-2 b_{i}^{l} m_{i}+b_{i}^{u 2} M_{i}^{2}+\sum_{j=1}^{n} g_{j i}^{u 2} M_{i}^{2}\right.\right. \\
+\sum_{j=1, j \neq i}^{n} c_{j i}^{u 2}+\left(1+2 b_{i}^{u} M_{i}\right) c_{i j}^{u}+\frac{1}{2} \sum_{l=1, l \neq i, j}^{n} c_{l i}^{u} c_{l j}^{u}
\end{array} \\
& \begin{array}{l}
\Delta V_{(47)}(k) \\
\leq \sum_{i=1}^{n}\left\{\left(-2 b_{i}^{l} m_{i}+b_{i}^{u 2} M_{i}^{2}+\sum_{j=1}^{n} g_{j i}^{u 2} M_{i}^{2}\right.\right. \\
+\sum_{j=1, j \neq i}^{n} c_{j i}^{u 2}+\left(1+2 b_{i}^{u} M_{i}\right) c_{i j}^{u}+\frac{1}{2} \sum_{l=1, l \neq i, j}^{n} c_{l i}^{u} c_{l j}^{u}
\end{array} \\
& +\frac{1}{2} \sum_{l=1}^{n} g_{l i}^{u} g_{l j}^{u} M_{i} M_{j}+e_{i}^{u}\left(b_{i}^{u} M_{i}+1\right) \\
& \left.+g_{i i}^{u} M_{i}\left(1-f_{i}^{l}\right)\right)\left(p_{i}(k)-q_{i}(k)\right)^{2} \\
& +\left(\left[e_{i}^{u}\left(b_{i}^{u} M_{i}+1\right)+g_{i i}^{u} M_{i}\left(1-f_{i}^{l}\right)\right]\right. \\
& +\left[e_{i}^{u 2}+f_{i}^{l}\left(f_{i}^{u}-2\right)\right] \\
& \left.+\sum_{j=1, j \neq i}^{n}\left[\left(1-f_{i}^{l}\right) g_{i j}^{u} M_{j}+e_{i}^{u} c_{i j}^{u}\right]\right) \\
& \begin{array}{l}
\times\left(u_{i}(k)-w_{i}(k)\right)^{2} \\
+\sum_{j=1, j \neq i}^{n}\left(\left(1+2 b_{i}^{u} M_{i}\right) c_{i j}^{u}+\frac{1}{2} \sum_{l=1, l \neq i, j}^{n} c_{l i}^{u} c_{l j}^{u}\right.
\end{array} \\
& V_{i 3}=2 \mid\left[e_{i}(k)\left(b_{i}(k) \xi_{i}(k)-1\right)+g_{i i}(k) \xi_{i}(k)\left(1-f_{i}(k)\right)\right] \\
& \times\left(p_{i}(k)-q_{i}(k)\right)\left(u_{i}(k)-w_{i}(k)\right) \\
& \begin{array}{l}
\times\left(u_{i}(k)-w_{i}(k)\right)^{2} \\
+\sum_{j=1, j \neq i}^{n}\left(\left(1+2 b_{i}^{u} M_{i}\right) c_{i j}^{u}+\frac{1}{2} \sum_{l=1, l \neq i, j}^{n} c_{l i}^{u} c_{l j}^{u}\right.
\end{array} \\
& \leq\left[e_{i}^{u}\left(b_{i}^{u} M_{i}+1\right)+g_{i i}^{u} M_{i}\left(1-f_{i}^{l}\right)\right] \\
& \times\left[\left(p_{i}(k)-q_{i}(k)\right)^{2}+\left(u_{i}(k)-w_{i}(k)\right)^{2}\right], \\
& \begin{array}{l}
+\frac{1}{2} \sum_{l=1}^{n} g_{l i}^{u} g_{l j}^{u} M_{i} M_{j} \\
\left.+\left(1-f_{i}^{l}\right) g_{i j}^{u} M_{j}+e_{i}^{u} c_{i j}^{u}\right)
\end{array} \\
& \begin{array}{l}
+\frac{1}{2} \sum_{l=1}^{n} g_{l i}^{u} g_{l j}^{u} M_{i} M_{j} \\
\left.+\left(1-f_{i}^{l}\right) g_{i j}^{u} M_{j}+e_{i}^{u} c_{i j}^{u}\right)
\end{array} \\
& V_{i 4}=2 \mid \sum_{j=1, j \neq i}^{n}\left[\left(1-f_{i}(k)\right) g_{i j}(k) \xi_{j}(k)\right. \\
& \left.-e_{i}(k) \frac{c_{i j}(k) d_{i j}(k) \xi_{j}(k)}{\left(d_{i j}(k)+e^{p_{j}(k)}\right)\left(d_{i j}(k)+e^{q_{j}(k)}\right)}\right] \\
& \times\left(p_{j}(k)-q_{j}(k)\right)\left(u_{i}(k)-w_{i}(k)\right) \\
& \leq \sum_{j=1, j \neq i}^{n}\left[\left(1-f_{i}^{l}\right) g_{i j}^{u} M_{j}+e_{i}^{u} c_{i j}^{u}\right] \\
& \times\left[\left(p_{j}(k)-q_{j}(k)\right)^{2}+\left(u_{i}(k)-w_{i}(k)\right)^{2}\right], \\
& \left.\times\left(p_{j}(k)-q_{j}(k)\right)^{2}\right\} \\
& =\sum_{i=1}^{n}\left\{\left(-2 b_{i}^{l} m_{i}+b_{i}^{u 2} M_{i}^{2}+\sum_{j=1}^{n} g_{j i}^{u 2} M_{i}^{2}\right.\right. \\
& +\sum_{j=1, j \neq i}^{n} c_{j i}^{u 2}+\left(1+2 b_{i}^{u} M_{i}\right) c_{i j}^{u} \\
& +\frac{1}{2} \sum_{l=1, l \neq i, j}^{n} c_{l i}^{u} c_{l j}^{u}+\frac{1}{2} \sum_{l=1}^{n} g_{l i}^{u} g_{l j}^{u} M_{i} M_{j}
\end{aligned}
$$




$$
\begin{aligned}
& \left.+e_{i}^{u}\left(b_{i}^{u} M_{i}+1\right)+g_{i i}^{u} M_{i}\left(1-f_{i}^{l}\right)\right) \\
& \times\left(p_{i}(k)-q_{i}(k)\right)^{2} \\
& +\left(e_{i}^{u}\left(b_{i}^{u} M_{i}+1\right)+g_{i i}^{u} M_{i}\left(1-f_{i}^{l}\right)\right. \\
& +e_{i}^{u 2}+f_{i}^{l}\left(f_{i}^{u}-2\right) \\
& \left.+\sum_{j=1, j \neq i}^{n}\left[\left(1-f_{i}^{l}\right) g_{i j}^{u} M_{j}+e_{i}^{u} c_{i j}^{u}\right]\right) \\
& \times\left(u_{i}(k)-w_{i}(k)\right)^{2} \\
& +\sum_{j=1, j \neq i}^{n}\left(\left(1+2 b_{j}^{u} M_{j}\right) c_{j i}^{u}+\frac{1}{2} \sum_{l=1, l \neq i, j}^{n} c_{l i}^{u} c_{l j}^{u}\right. \\
& +\frac{1}{2} \sum_{l=1}^{n} g_{l i}^{u} g_{l j}^{u} M_{i} M_{j} \\
& \left.+\left(1-f_{j}^{l}\right) g_{j i}^{u} M_{i}+e_{j}^{u} c_{j i}^{u}\right) \\
& \left.\times\left(p_{i}(k)-q_{i}(k)\right)^{2}\right\} \\
& =-\sum_{i=1}^{n}\left\{\left(2 b_{i}^{l} m_{i}-b_{i}^{u 2} M_{i}^{2}-\sum_{j=1}^{n} g_{j i}^{u 2} M_{i}^{2}\right.\right. \\
& -\sum_{j=1, j \neq i}^{n} c_{j i}^{u 2}-\left(1+2 b_{i}^{u} M_{i}\right) c_{i j}^{u} \\
& -\frac{1}{2} \sum_{l=1, l \neq i, j}^{n} c_{l i}^{u} c_{l j}^{u}-\frac{1}{2} \sum_{l=1}^{n} g_{l i}^{u} g_{l j}^{u} M_{i} M_{j} \\
& -e_{i}^{u}\left(b_{i}^{u} M_{i}+1\right)-g_{i i}^{u} M_{i}\left(1-f_{i}^{l}\right) \\
& -\sum_{j=1, j \neq i}^{n}\left[\left(1+2 b_{j}^{u} M_{j}\right) c_{j i}^{u}+\frac{1}{2} \sum_{l=1, l \neq i, j}^{n} c_{l i}^{u} c_{l j}^{u}\right. \\
& +\frac{1}{2} \sum_{l=1}^{n} g_{l i}^{u} g_{l j}^{u} M_{i} M_{j} \\
& \left.\left.+\left(1-f_{j}^{l}\right) g_{j i}^{u} M_{i}+e_{j}^{u} c_{j i}^{u}\right]\right) \\
& \times\left(p_{i}(k)-q_{i}(k)\right)^{2} \\
& +\left(f_{i}^{l}\left(2-f_{i}^{u}\right)-e_{i}^{u}\left(b_{i}^{u} M_{i}+1\right)\right.
\end{aligned}
$$

where $\beta=\min \left\{\min _{1 \leq i \leq n} \beta_{i}^{1}, \min _{1 \leq i \leq n} \beta_{i}^{2}\right\}$. That is, there exists a positive constant $0<\beta<1$ such that

$$
\Delta V_{(47)}(k, Q, W) \leq-\beta V(k, Q, W) .
$$

From $0<\beta<1$, the condition (iii) of Theorem 9 is satisfied. Then, according to Theorem 9, there exists a unique uniformly asymptotically stable almost periodic solution $\left(p_{1}(k), p_{2}(k), \ldots, p_{n}(k), u_{1}(k), u_{2}(k), \ldots, u_{n}(k)\right) \quad$ of $\quad(45)$ which is bounded by $S^{*}$ for all $k \in Z^{+}$. It means that there exists a unique uniformly asymptotically stable almost periodic solution $\left(x_{1}(k), x_{2}(k), \ldots, x_{n}(k), u_{1}(k), u_{2}(k), \ldots, u_{n}(k)\right)$ of system (1) which is bounded by $\Omega$ for all $k \in Z^{+}$. This completed the proof.

Remark 13. If $n=2$, the conditions of Theorem 12 can be simplified. Therefore, we have the following result.

Corollary 14. Let $n=2$, and assume further that $0<\beta<1$, where

$$
\begin{gathered}
\beta=\min \left\{\beta_{12}^{1}, \beta_{21}^{1}, \beta_{12}^{2}, \beta_{21}^{2}\right\} \\
\beta_{i j}^{1}=2 b_{i}^{l} m_{i}-b_{i}^{u 2} M_{i}^{2}-\sum_{l=1}^{2} g_{l i}^{u 2} M_{i}^{2}-c_{j i}^{u 2} \\
-\left(1+2 b_{i}^{u} M_{i}\right) c_{i j}^{u}-\sum_{l=1}^{2} g_{l i}^{u} g_{l j}^{u} M_{i} M_{j} \\
-e_{i}^{u}\left(b_{i}^{u} M_{i}+1\right)-g_{i i}^{u} M_{i}\left(1-f_{i}^{l}\right) \\
-\left(1+2 b_{j}^{u} M_{j}\right) c_{j i}^{u}-\left(1-f_{j}^{l}\right) g_{j i}^{u} M_{i}-e_{j}^{u} c_{j i}^{u}, \\
\beta_{i j}^{2}=f_{i}^{l}\left(2-f_{i}^{u}\right)-e_{i}^{u}\left(b_{i}^{u} M_{i}+1\right) \\
-g_{i i}^{u} M_{i}\left(1-f_{i}^{l}\right)-e_{i}^{u 2}-\left[\left(1-f_{i}^{l}\right) g_{i j}^{u} M_{j}+e_{i}^{u} c_{i j}^{u}\right],
\end{gathered}
$$

$i, j=1,2, j \neq i$. Then system (1) admits a unique uniformly asymptotically stable almost periodic solution $\left(x_{1}(k)\right.$, $\left.x_{2}(k), u_{1}(k), u_{2}(k)\right)$ which is bounded by $\Omega$ for all $k \in Z^{+}$. 


\section{Example and Numerical Simulation}

In this section, we give the following example to check the feasibility of our result.

Example 15. Consider the following almost periodic discrete Lotka-Volterra mutualism system with feedback controls:

$$
\begin{aligned}
& x_{1}(k+1) \\
& =x_{1}(k) \\
& \times \exp \{1.2-0.02 \sin (\sqrt{2} k) \\
& -(1.05+0.01 \sin (\sqrt{2} k)) x_{1}(k) \\
& +\frac{(0.025+0.002 \cos (\sqrt{2} k)) x_{2}(k)}{0.2+0.003 \cos (\sqrt{2} k)+x_{2}(k)} \\
& +\frac{(0.02+0.001 \cos (\sqrt{2} k)) x_{3}(k)}{0.4+0.03 \cos (\sqrt{2} k)+x_{3}(k)} \\
& \left.-(0.025+0.002 \cos (\sqrt{3} k)) u_{1}(k)\right\} \text {, } \\
& x_{2}(k+1) \\
& =x_{2}(k) \\
& \times \exp \{1.1-0.025 \sin (\sqrt{2} k) \\
& -(1.08+0.015 \sin (\sqrt{2} k)) x_{2}(k) \\
& +\frac{(0.02+0.003 \cos (\sqrt{2} k)) x_{1}(k)}{0.3+0.02 \cos (\sqrt{3} k)+x_{1}(k)} \\
& +\frac{(0.025+0.002 \cos (\sqrt{2} k)) x_{3}(k)}{0.2+0.07 \sin (\sqrt{2} k)+x_{3}(k)} \\
& \left.-(0.025+0.002 \sin (\sqrt{2} k)) u_{2}(k)\right\} \text {, } \\
& x_{3}(k+1) \\
& =x_{3}(k) \\
& \times \exp \{1.15-0.03 \sin (\sqrt{2} k) \\
& -(1.1+0.002 \sin (\sqrt{2} k)) x_{3}(k) \\
& +\frac{(0.03+0.0025 \cos (\sqrt{2} k)) x_{1}(k)}{0.2+0.004 \sin (\sqrt{2} k)+x_{1}(k)}
\end{aligned}
$$

$$
\begin{aligned}
& +\frac{(0.028+0.0015 \cos (\sqrt{2} k)) x_{2}(k)}{0.2+0.004 \cos (\sqrt{3} k)+x_{2}(k)} \\
& \left.-(0.025+0.002 \cos (\sqrt{2} k)) u_{3}(k)\right\},
\end{aligned}
$$

$$
\begin{aligned}
\Delta u_{1}(k)= & -(0.925-0.03 \sin (\sqrt{2} k)) u_{1}(k) \\
& +(0.015-0.005 \sin (\sqrt{2} k)) x_{1}(k) \\
& +(0.013-0.004 \sin (\sqrt{3} k)) x_{2}(k) \\
& +(0.02-0.006 \cos (\sqrt{2} k)) x_{3}(k)
\end{aligned}
$$$$
\Delta u_{2}(k)=-(0.925-0.04 \sin (\sqrt{3} k)) u_{2}(k)
$$$$
+(0.016-0.004 \sin (\sqrt{3} k)) x_{1}(k)
$$$$
+(0.015-0.005 \cos (\sqrt{2} k)) x_{2}(k)
$$$$
+(0.014-0.004 \sin (\sqrt{2} k)) x_{3}(k) \text {, }
$$$$
\Delta u_{3}(k)=-(0.935-0.035 \cos (\sqrt{2} k)) u_{3}(k)
$$$$
+(0.017-0.006 \cos (\sqrt{2} k)) x_{1}(k)
$$$$
+(0.013-0.005 \sin (\sqrt{3} k)) x_{2}(k)
$$$$
+(0.016-0.005 \cos (\sqrt{2} k)) x_{3}(k) \text {. }
$$

By simple computation, we derive

$$
\begin{aligned}
& \beta_{1}^{1} \approx 0.0092, \quad \beta_{2}^{1} \approx 0.0042, \quad \beta_{3}^{1} \approx 0.0014, \\
& \beta_{1}^{2} \approx 0.0031, \quad \beta_{2}^{2} \approx 0.0081, \quad \beta_{3}^{2} \approx 0.0072 .
\end{aligned}
$$

Then

$$
0<\beta=\min \left\{\beta_{1}^{1}, \beta_{2}^{1}, \beta_{3}^{1}, \beta_{1}^{2}, \beta_{2}^{2}, \beta_{3}^{2}\right\}<1 .
$$

Also it is easy to see that the conditions of Theorem 12 are verified. Therefore, system (60) has a unique positive almost periodic solution which is uniformly asymptotically stable. Our numerical simulations support our results (see Figure 1).

\section{Conflict of Interests}

The authors declare that there is no conflict of interests regarding the publication of this paper, and there are no financial conflict of interests between the authors and the commercial identity.

\section{Acknowledgments}

The authors are grateful to the anonymous referees for their excellent suggestions, which greatly improved the 

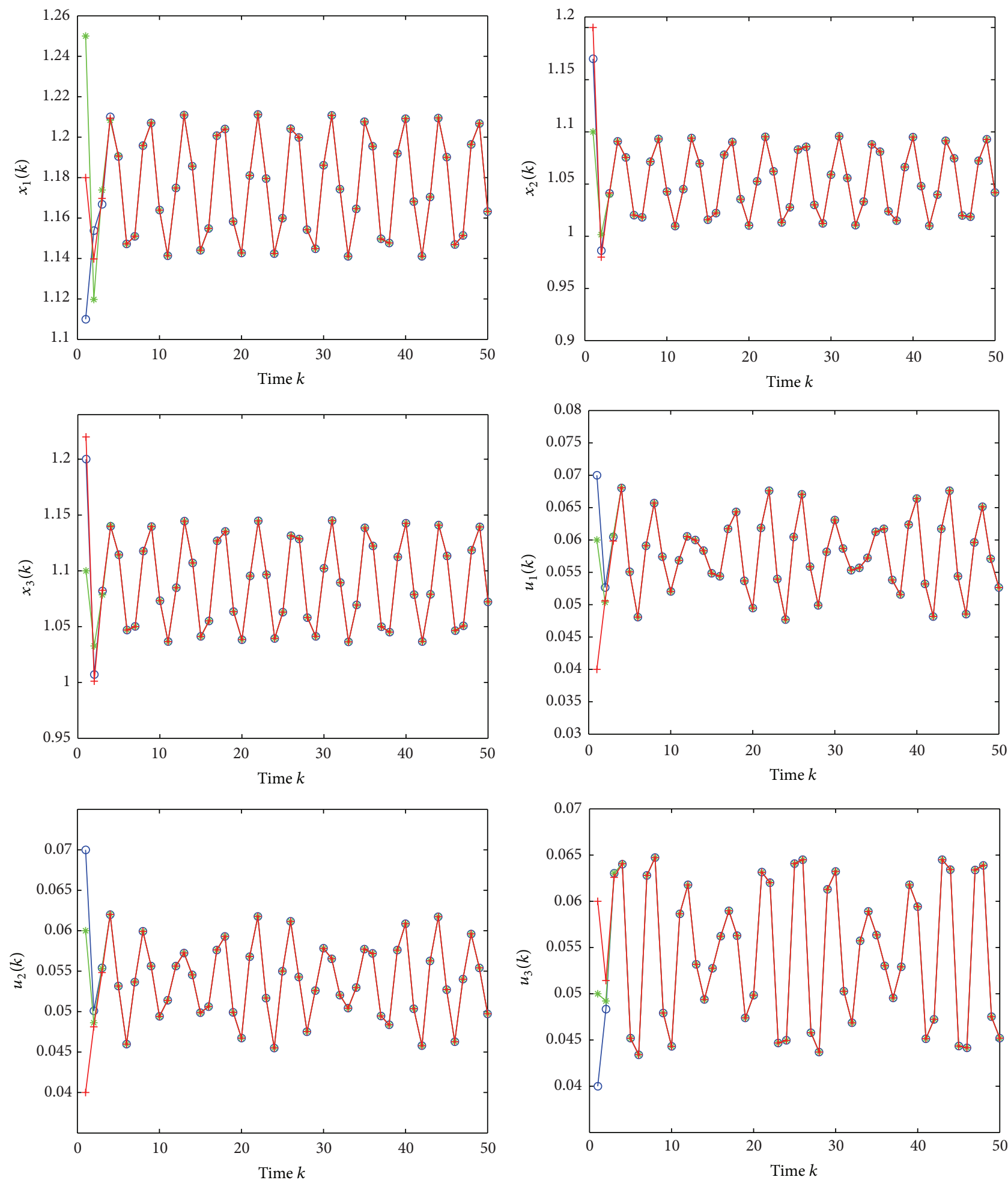

FIGURE 1: Dynamic behavior of positive almost periodic solution $\left(x_{1}(k), x_{2}(k), x_{3}(k), u_{1}(k), u_{2}(k), u_{3}(k)\right)$ of system $(60)$ with the three initial conditions $(1.11,1.17,1.2,0.07,0.07,0.04),(1.25,1.1,1.1,0.06,0.06,0.05)$, and $(1.18,1.2,1.22,0.04,0.04,0.06)$ for $k \in[1,50]$, respectively.

presentation of the paper. Also, this work is supported by National Natural Science Foundation of China (nos. 61132008 and 11301415), Natural Science Basic Research Plan in Shaanxi
Province of China (no. 2013JQ1020) and Scientific Research Program Funded by Shaanxi Provincial Education Department of China (no. 2013JK1098). 


\section{References}

[1] C. L. Wolin and L. R. Lawlor, "Models of facultative mutualism: density effects," The American Naturalist, vol. 124, pp. 843-862, 1984.

[2] Y. Xia, J. Cao, and S. S. Cheng, "Periodic solutions for a Lotka-Volterra mutualism system with several delays," Applied Mathematical Modelling, vol. 31, no. 9, pp. 1960-1969, 2007.

[3] Y. Li and H. Zhang, "Existence of periodic solutions for a periodic mutualism model on time scales," Journal of Mathematical Analysis and Applications, vol. 343, no. 2, pp. 818-825, 2008.

[4] Y. Wang, "Asymptotic behavior of solutions for a Lotka-Volterra mutualism reaction-diffusion system with time delays," Computers \& Mathematics with Applications, vol. 58, no. 3, pp. 597604, 2009.

[5] C. Wang, S. Wang, F. Yang, and L. Li, "Global asymptotic stability of positive equilibrium of three-species Lotka-Volterra mutualism models with diffusion and delay effects," Applied Mathematical Modelling, vol. 34, no. 12, pp. 4278-4288, 2010.

[6] M. Liu and K. Wang, "Analysis of a stochastic autonomous mutualism model," Journal of Mathematical Analysis and Applications, vol. 402, no. 1, pp. 392-403, 2013.

[7] H. Zhang, Y. Li, B. Jing, and W. Zhao, "Global stability of almost periodic solution of multispecies mutualism system with time delays and impulsive effects," Applied Mathematics and Computation, vol. 232, pp. 1138-1150, 2014.

[8] Y. Li, "Positive periodic solutions of a discrete mutualism model with time delays," International Journal of Mathematics and Mathematical Sciences, no. 4, pp. 499-506, 2005.

[9] F. Chen, "Permanence for the discrete mutualism model with time delays," Mathematical and Computer Modelling, vol. 47, no. 3-4, pp. 431-435, 2008.

[10] X. Li and W. Yang, "Permanence of a discrete model of mutualism with infinite deviating arguments," Discrete Dynamics in Nature and Society, vol. 2010, Article ID 931798, 7 pages, 2010.

[11] Z. Wang and Y. Li, "Almost periodic solutions of a discrete mutualism model with feedback controls," Discrete Dynamics in Nature and Society, vol. 2010, Article ID 286031, 18 pages, 2010.

[12] H. Zhang, Y. Li, and B. Jing, "Global attractivity and almost periodic solution of a discrete mutualism model with delays," Mathematical Methods in the Applied Sciences, 2013.

[13] H. Zhang, B. Jing, Y. Li, and X. Fang, "Global analysis of almost periodic solution of a discrete multispecies mutualism system," Journal of Applied Mathematics, vol. 2014, Article ID 107968, 12 pages, 2014.

[14] F. Chen, "Permanence and global attractivity of a discrete multispecies Lotka-Volterra competition predator-prey systems," Applied Mathematics and Computation, vol. 182, no. 1, pp. 3-12, 2006.

[15] F. Chen, "Permanence of a discrete $N$-species cooperation system with time delays and feedback controls," Applied Mathematics and Computation, vol. 186, no. 1, pp. 23-29, 2007.

[16] C. Wang and J. Shi, "Periodic solution for a delay multispecies logarithmic population model with feedback control," Applied Mathematics and Computation, vol. 193, no. 1, pp. 257-265, 2007.

[17] N. Fang and X. X. Chen, "Permanence of a discrete multispecies Lotka-Volterra competition predator-prey system with delays," Nonlinear Analysis: Real World Applications, vol. 9, no. 5, pp. 2185-2195, 2008.

[18] M. He and F. Chen, "Dynamic behaviors of the impulsive periodic multi-species predator-prey system," Computers \& Mathematics with Applications, vol. 57, no. 2, pp. 248-265, 2009.
[19] W. Yang and X. Li, "Permanence of a discrete nonlinear $N$ species cooperation system with time delays and feedback controls," Applied Mathematics and Computation, vol. 218, no. 7, pp. 3581-3586, 2011.

[20] Z. Cai, L. Huang, and H. Chen, "Positive periodic solution for a multispecies competition-predator system with Holling III functional response and time delays," Applied Mathematics and Computation, vol. 217, no. 10, pp. 4866-4878, 2011.

[21] Y. Li and T. Zhang, "Permanence of a discrete $n$-species cooperation system with time-varying delays and feedback controls," Mathematical and Computer Modelling, vol. 53, no. 56, pp. 1320-1330, 2011.

[22] Y. Wang, "Periodic and almost periodic solutions of a nonlinear single species discrete model with feedback control," Applied Mathematics and Computation, vol. 219, no. 10, pp. 5480-5486, 2013.

[23] T. Zhang and X. Gan, "Almost periodic solutions for a discrete fishing model with feedback control and time delays," Communications in Nonlinear Science and Numerical Simulation, vol. 19, no. 1, pp. 150-163, 2014.

[24] Z. Du and Y. Lv, "Permanence and almost periodic solution of a Lotka-Volterra model with mutual interference and time delays," Applied Mathematical Modelling, vol. 37, no. 3, pp. 10541068, 2013.

[25] L. Wang, M. Yu, and P. Niu, "Periodic solution and almost periodic solution of impulsive Lasota-Wazewska model with multiple time-varying delays," Computers \& Mathematics with Applications, vol. 64, no. 8, pp. 2383-2394, 2012.

[26] B. Yang and J. Li, "An almost periodic solution for an impulsive two-species logarithmic population model with time-varying delay," Mathematical and Computer Modelling, vol. 55, no. 7-8, pp. 1963-1968, 2012.

[27] J. O. Alzabut, G. T. Stamov, and E. Sermutlu, "Positive almost periodic solutions for a delay logarithmic population model," Mathematical and Computer Modelling, vol. 53, no. 1-2, pp. 161167, 2011.

[28] Q. Wang, H. Zhang, and Y. Wang, "Existence and stability of positive almost periodic solutions and periodic solutions for a logarithmic population model," Nonlinear Analysis, vol. 72, no. 12, pp. 4384-4389, 2010.

[29] F. Chen and X. Cao, "Existence of almost periodic solution in a ratio-dependent Leslie system with feedback controls," Journal of Mathematical Analysis and Applications, vol. 341, no. 2, pp. 1399-1412, 2008.

[30] C. Niu and X. Chen, "Almost periodic sequence solutions of a discrete Lotka-Volterra competitive system with feedback control," Nonlinear Analysis: Real World Applications, vol. 10, no. 5, pp. 3152-3161, 2009.

[31] Z. Zhao, T. Wang, and L. Chen, "Dynamic analysis of a turbidostat model with the feedback control," Communications in Nonlinear Science and Numerical Simulation, vol. 15, no. 4, pp. 1028-1035, 2010.

[32] W. Yang and X. Li, "Feedback control has no influence on the persistent property of a single species discrete model with time delays," Mathematical and Computer Modelling, vol. 54, no. 9-10, pp. 2007-2013, 2011.

[33] Y. Li and T. Zhang, "Permanence and almost periodic sequence solution for a discrete delay logistic equation with feedback control," Nonlinear Analysis: Real World Applications, vol. 12, no. 3, pp. 1850-1864, 2011.

[34] T. Zhang, Y. Li, and Y. Ye, "Persistence and almost periodic solutions for a discrete fishing model with feedback control," 
Communications in Nonlinear Science and Numerical Simulation, vol. 16, no. 3, pp. 1564-1573, 2011.

[35] A. M. Fink, Almost Periodic Differential Equations, vol. 377 of Lecture Notes in Mathematics, Springer, Berlin, Germany, 1974.

[36] A. M. Fink and G. Seifert, "Liapunov functions and almost periodic solutions for almost periodic systems," Journal of Differential Equations, vol. 5, pp. 307-313, 1969.

[37] Y. Hamaya, "Existence of an almost periodic solution in a difference equation by Liapunov functions," Nonlinear Studies, vol. 8, no. 3, pp. 373-379, 2001.

[38] S. Zhang, "Existence of almost periodic solutions for difference systems," Annals of Differential Equations, vol. 16, no. 2, pp. 184206, 2000.

[39] R. Yuan and J. Hong, "The existence of almost periodic solutions for a class of differential equations with piecewise constant argument," Nonlinear Analysis: Theory, Methods \& Applications, vol. 28, no. 8, pp. 1439-1450, 1997.

[40] Y. Fan and L. Wang, "Permanence for a discrete model with feedback control and delay," Discrete Dynamics in Nature and Society, vol. 2008, Article ID 945109, 8 pages, 2008. 


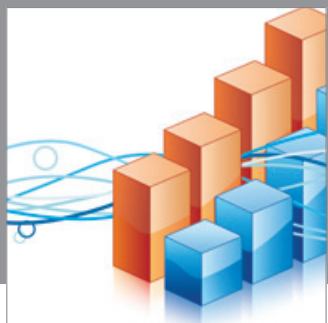

Advances in

Operations Research

mansans

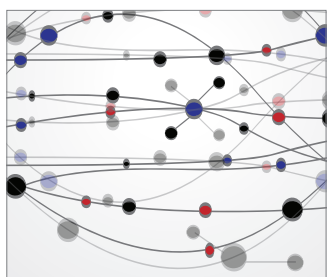

The Scientific World Journal
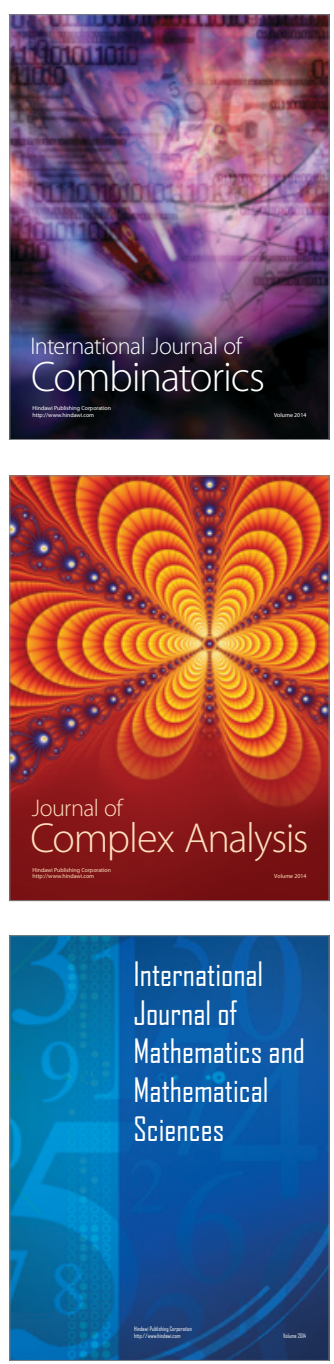
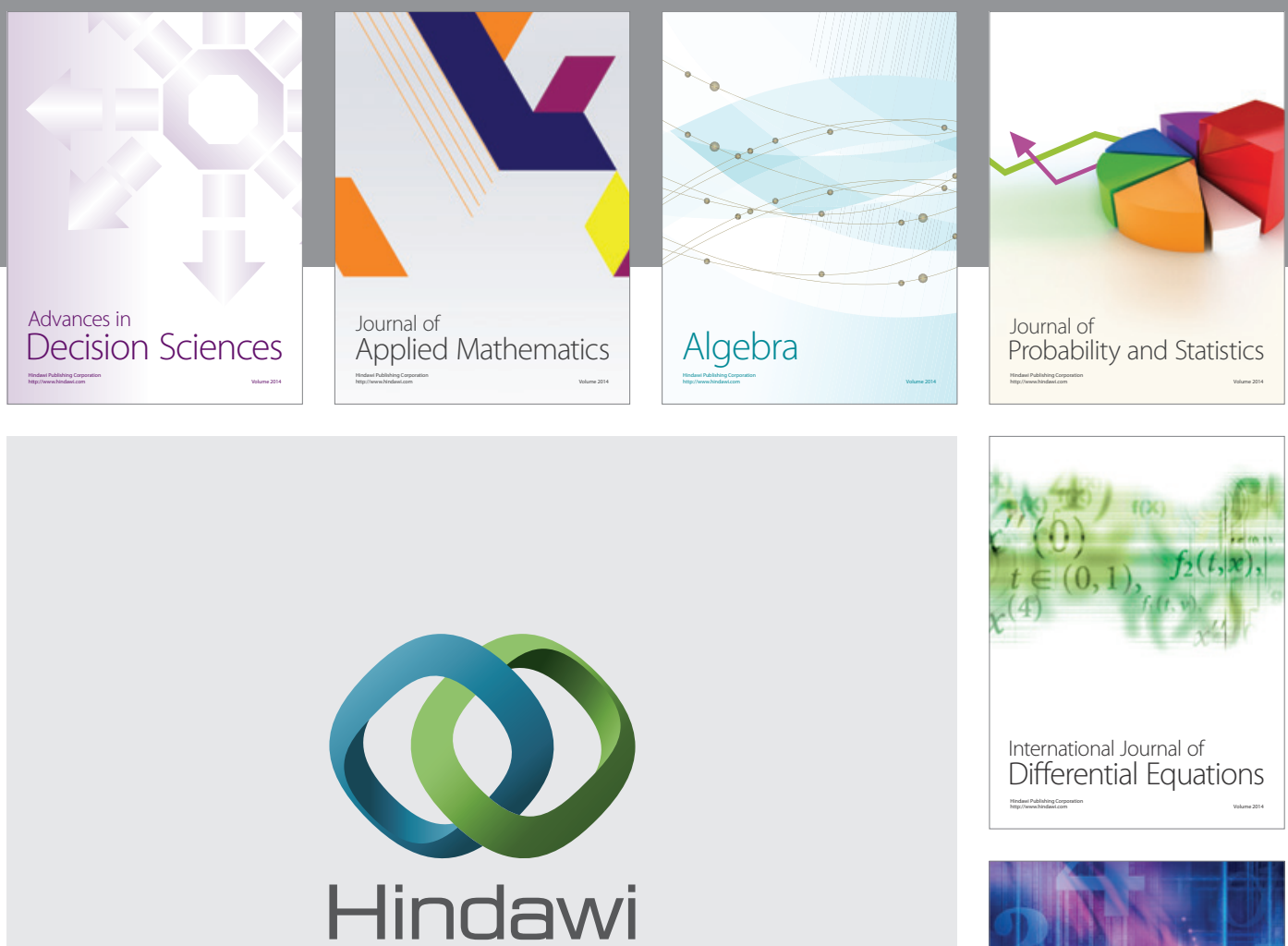

Submit your manuscripts at http://www.hindawi.com
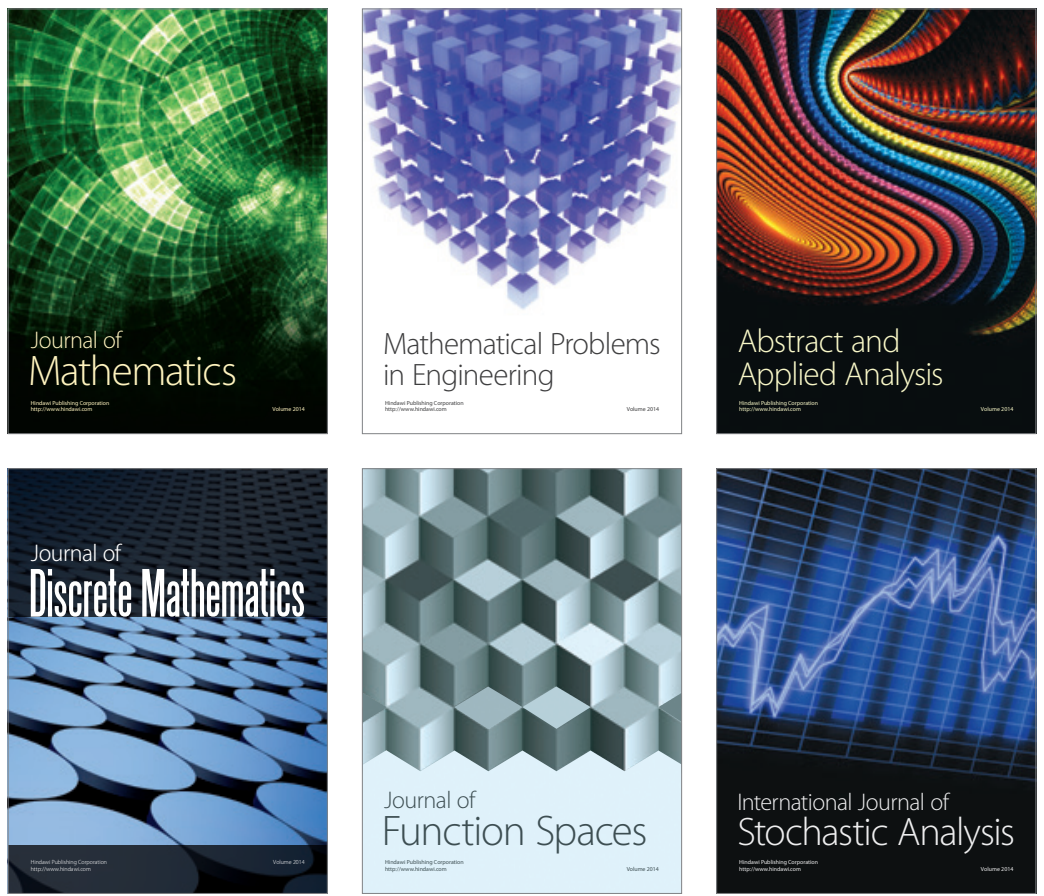

Journal of

Function Spaces

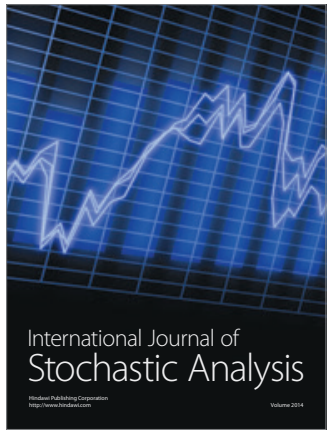

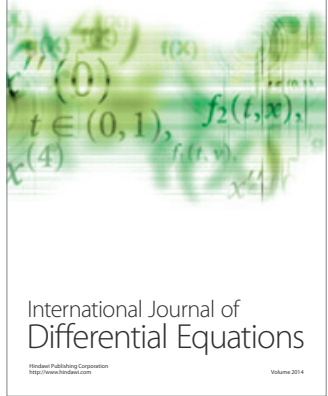
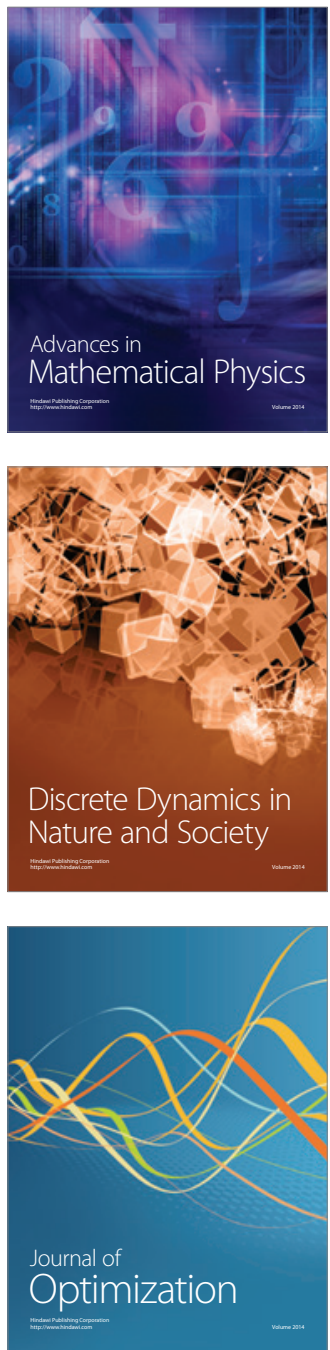